\title{
Matching NLO QCD with parton shower in Monte Carlo scheme - the KrkNLO method ${ }^{1}$
}

\author{
S. Jadach, ${ }^{a}$ W. Płaczek, ${ }^{b}$ S. Sapeta, ${ }^{c}$ A. Siódmok ${ }^{a, c}$ and M. Skrzypek ${ }^{a}$ \\ ${ }^{a}$ Institute of Nuclear Physics, Polish Academy of Sciences, \\ ul. Radzikowskiego 152, 31-342 Kraków, Poland \\ ${ }^{b}$ Marian Smoluchowski Institute of Physics, Jagiellonian University, \\ ul. Eojasiewicza 11, 30-348 Kraków, Poland \\ ${ }^{c}$ Theory Unit (PH-TH), CERN, \\ CH-1211, Geneva 23, Switzerland \\ E-mail: Stanislaw.Jadach@cern.ch, Wieslaw.Placzek@uj.edu.pl, \\ sebastian.sapeta@cern.ch, andrzej@cern.ch, Maciej.Skrzypek@ifj.edu.pl
}

ABSTRACT: A new method of including the complete NLO QCD corrections to hard processes in the LO parton-shower Monte Carlo (PSMC) is presented. This method, called KrkNLO, requires the use of parton distribution functions in a dedicated Monte Carlo (MC) factorization scheme, which is also discussed in this paper. In the future, it may simplify introduction of the NNLO corrections to hard processes and the NLO corrections to PSMC. Details of the method and numerical examples of its practical implementation as well as comparisons with other calculations, such as MCFM, MC@NLO, POWHEG, for single $Z / \gamma^{*}$-boson production at the LHC are presented.

Keywords: Monte Carlo Simulations, NLO Computations

ARXIV EPRINT: 1503.06849

\footnotetext{
${ }^{1}$ This work is partly supported by the Polish National Science Center grant DEC-2011/03/B/ST2/02632, the Polish National Science Centre grant UMO-2012/04/M/ST2/00240.
} 


\section{Contents}

1 Introduction 1

2 Kinematics and phase space parametrization 3

2.1 Single emission 3

$\begin{array}{lll}2.2 & \text { Multiple emissions } & 7\end{array}$

$\begin{array}{llr}3 & \text { KrkNLO methodology } & 10\end{array}$

$3.1 \quad$ NLO-correcting weight 11

$\begin{array}{lll}3.2 & \text { MC factorization scheme } & 13\end{array}$

$\begin{array}{lll}3.3 & \text { Multiparton reality of PSMC } & 15\end{array}$

$\begin{array}{ll}\text { 3.4 NLO weight in parton shower and its algebraic validation } & 19\end{array}$

3.5 Summarizing the KrkNLO method 22

4 Fixed-order NLO benchmarks $\quad 24$

$\begin{array}{lll}\text { 4.1 PDFs in MC scheme } & 25\end{array}$

4.2 $\mathrm{MC}$ and $\overline{\mathrm{MS}}$ schemes at NLO 27

5 Results for NLO with parton shower $\quad 30$

5.1 Initial results for $q \bar{q}$ channel only 31

5.2 All channels 33

$\begin{array}{llr}6 & \text { Summary and outlook } & 38\end{array}$

$\begin{array}{lll}\text { A First emission in backward evolution } & 39\end{array}$

B Exclusive NLO corrections in $\overline{\mathrm{MS}}$ scheme 41

\section{Introduction}

Higher-order perturbative corrections in Quantum Chromodynamics (QCD), important for the LHC data analysis, are calculated order by order in the strong coupling, $\alpha_{s}$, while some numerically important ones, related to soft and collinear singularities, can be resumed to the infinite order. The most valuable, albeit technically difficult, way of QCD resummation is in form of a Monte Carlo (MC) event generator [1]. It is widely recognized that the most promising way of getting high precision QCD calculation for hadron collider data analysis is a common framework of fixed-order calculations combined with a parton shower Monte Carlo (PSMC). The pioneering work, in which the complete first-order QCD corrections to the hard process of heavy boson production in hadron-hadron collision were combined with PSMC, was that of ref. [2]. Shortly later another interesting variant was proposed in ref. [3]. Presently both methods are available for many processes, see ref. [4]. 
It is worth to mention that partial efforts in this direction were done earlier in the course of development of the most popular PSMCs, like for instance in ref. [5] or [6] and in many other works. In these earlier attempts, the distributions generated in PSMC were improved using a tree-level exact matrix element (ME) of QCD, while virtual corrections were neglected or taken in the leading-logarithmic approximation [7]. It was also known for a long time, that an ad hoc approach, in which PSMC differential distributions were corrected using the exact ME and the overall normalization was corrected by hand to fixedorder next-to-leading (NLO) integrated cross section, was providing distributions in a quite good agreement with experimental data. It may be therefore a little bit surprising that it took two decades to work out a systematic method of combining the NLO-corrections to the hard process, known from the early 1980's, see for instance ref. [8], with the leadingorder (LO) PSMC, also dating from the early 1980's. Apart from the lack of interest in more precise QCD calculations due to poor data quality, main reasons for this much delayed development can be seen from problems addressed in refs. [2, 3]. Namely, any such a method requires a very good NLO-level analytic understanding/control of distributions from PSMC and, either NLO-level complete phase space coverage for the hard process or a practical methodology of correcting for the lack of it. Luckily, a new wave of developments of the LO parton showers, see refs. [9-12], has lead to modernized PSMCs, better suited for merging/matching with the fixed-order QCD calculations, in particular with better or even complete coverage of the hard process phase space.

It is now obvious that the next challenge on the way to even higher-precision perturbative QCD calculations needed until the end of the LHC era two decades from now, is to combine the fully exclusive NNLO corrections to the hard process and the NLO parton shower. The fixed-order NNLO corrections to many processes are already well established, see for instance refs. [13, 14], but the NLO PSMC needed for such a progress is still not available, except of feasibility studies summarized in ref. [15]. Interesting partial solution of combining the NNLO-corrected hard process with the LO parton shower can be found in $^{1}$ refs. [16-18]. The present work is relevant for the above future developments it the sense that it presents a simplified method of correcting the hard process to the NLO level in combination with the LO parton shower (PS). In other words, it offers a simpler alternative to the MC@NLO and POWHEG methods of refs. [2,3], which may hopefully pave the way to the NNLO hard process combined with NLO PSMC.

The new method described here, nicknamed as KrkNLO, was already proposed in ref. [19], where its first numerical implementation was done on top of the dedicated toy model PSMC and was limited to the gluonstrahlung subset of the NLO corrections. Later on it was tested numerically in a more detail in refs. $[15,20]$. In the present work the KrkNLO method is implemented within the standard PSMC Sherpa 2.0.0 [11]. A pilot study of KrkNLO implementation outside PS MC, using MC event encoded in the event records produced by Herwig $++2.7 .0[9,21,22]$ and Sherpa 2.0.0 was also done. Let us stress, however, that the overall simplifications of the KrkNLO method comes not completely for free, as it requires to use parton distribution functions (PDFs) in a special Monte Carlo

\footnotetext{
${ }^{1}$ In these methods only certain selected important distributions are upgraded to the NNLO level.
} 
(MC) factorization scheme (obtained, however, easily from reprocessing the $\overline{\mathrm{MS}} \mathrm{PDFs}$ ), and it is required that the basic LO PSMC provides for the NLO-complete coverage of the hard process phase space (this is also not a problem for all modern PSMCs). Our method is simpler to implement in the case of PSMC with an ordering based on the transverse momentum $k_{T}$ or a $q^{2}$ variable of ref. [23], inspired by the classic Catani-Seymour work [24]. However, it can be also easily implemented on top of PSMC that uses the angular ordering - without the need of the so-called truncated showers required in the POWHEG method, see refs. [20, 25] for more discussion on that.

The main advantage of the KrkNLO method is a simplification of the NLO corrections due to the use of PDFs in the MC factorization scheme. The implementation of the entire NLO corrections with the help of a single multiplicative simple weight on top of the LO distribution is a quite unique feature of the KrkNLO method.

Numerical studies presented here will be extended in the future publications to a wider range of distributions, energies, implementation variants, including comparisons with experimental data.

The paper is organized as follows. In section 2 we introduce the kinematics and the phase space parametrization for the considered process. In section 3 we describe in detail the KrkNLO method. Sections 4 and 5 contain some numerical results of the KrkNLO implementation: section 4 from the fixed-order NLO cross-checks while section 5 from the NLO+PSMC comparisons with the MCFM, MCQNLO and POWHEG programs for the main $Z$-boson observables. Section 6 summarizes the paper. In appendix A we add some details on the first gluon emission in the backward evolution in PSMC.

\section{Kinematics and phase space parametrization}

In the present work we are going to concentrate on the Drell-Yan process, specifically production and decay of the heavy boson ${ }^{2} Z / \gamma^{*}$ in proton-proton collisions. At the leading order (LO), $q \bar{q} \rightarrow Z$ is the only partonic subprocess that contributes. At the next-to-leading (NLO) level, the real correction $q \bar{q} \rightarrow Z g$ and the virtual correction to $q \bar{q} \rightarrow Z$ contributes - to be referred to collectively as $q \bar{q}$ channel. The remaining NLO contributions, $q g \rightarrow Z q$ and $\bar{q} g \rightarrow Z \bar{q}$, are tree-level only — to be called the $q g$ channel.

\subsection{Single emission}

Figure 1 illustrates part of the notation that will be used throughout the paper. The diagram shows the real correction to the $Z$-boson production in the $q \bar{q}$ channel with the gluon four-momentum denoted by $k_{1}$. The four-momenta of the incoming forward and backward partons, $p_{1 F}$ and $p_{1 B}$ are related to the four momenta of the incoming protons, $P_{F}$ and $P_{B}$, as follows

$$
p_{0 F}=x_{F} P_{F}, \quad p_{0 B}=x_{B} P_{B},
$$

\footnotetext{
${ }^{2}$ For brevity, in what follows, we shall often speak about the $Z$ boson only, but in all cases we really mean $Z / \gamma^{*} \rightarrow \ell^{+} \ell^{-}$.
} 


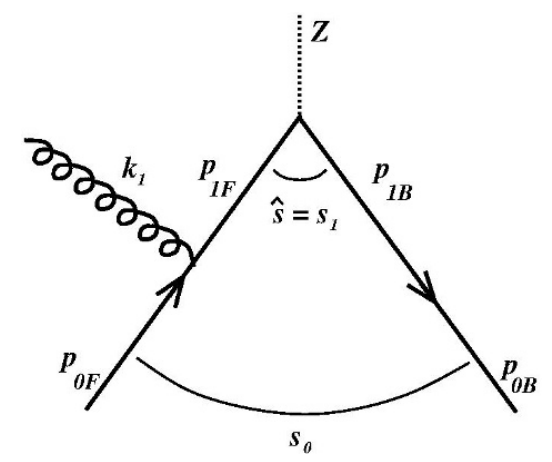

Figure 1. Kinematics of $Z$-boson production in the $q \bar{q}$ channel.

where

$$
P_{F}=\frac{\sqrt{s}}{2}\left(1,0_{T}, 1\right), \quad P_{B}=\frac{\sqrt{s}}{2}\left(1,0_{T},-1\right) .
$$

The invariant masses of the incoming parton pair prior to gluon emission and that of the produced $Z$ boson are denoted by $s_{0}$ and $\hat{s}=s_{1}$, respectively. Their ratio ${ }^{3}$

$$
z_{1}=\frac{s_{1}}{s_{0}}=\frac{\hat{s}}{s_{0}}
$$

can be related to light-cone variables of the emitted gluon, as seen from the kinematics of the emitted gluon expressed in terms of the light-cone Sudakov variables

$$
\begin{aligned}
& \alpha_{1}=\frac{2 k_{1} \cdot p_{0_{B}}}{s_{0}}=\frac{2 k_{1}^{+}}{s_{0}}, \quad \beta_{1}=\frac{2 k_{1} \cdot p_{0_{F}}}{s_{0}}=\frac{2 k_{1}^{-}}{s_{0}}, \quad \alpha_{1}+\beta_{1} \leq 1, \quad \alpha_{1}, \beta_{1} \geq 0, \\
& z_{1}=1-\alpha_{1}-\beta_{1}, \quad k_{1 T}^{2}=\alpha_{1} \beta_{1} s_{0}, \quad y_{1}=\frac{1}{2} \ln \frac{\alpha_{1}}{\beta_{1}}, \quad s_{0}=2 p_{0_{F}} p_{0_{B}},
\end{aligned}
$$

spanned on the four-momenta $p_{1_{F}}$ and $p_{1_{B}}$ of the incoming partons, prior to the gluon emission. As seen in eqs. (2.4), the variables $\alpha_{1}, \beta_{1}$ are simply the fractions of the light-cone components of the gluon four-momentum to the centre-of-mass (CM) energy of the incoming partons (prior to the gluon emission). The ratio $z_{1}$, the gluon transverse momentum $k_{1 T}$ and the gluon rapidity $y_{1}$ are related to the $\alpha_{1}, \beta_{1}$ variables as well.

In PSMC, the (eikonal) phase space measure of the emitted gluon

$$
\frac{d^{3} k_{1}}{2 k_{1}^{0}} \frac{1}{k_{1 T}^{2}}=\frac{\pi}{2} \frac{d \phi_{1}}{2 \pi} \frac{d \alpha_{1}}{\alpha_{1}} \frac{d \beta_{1}}{\beta_{1}}
$$

is always split one way or another into two parts belonging to the quark and antiquark emitters. A sharp division along the $y_{1}=0$ angular boundary was used in ref. [19], while in modern PSMCs, see refs. [9, 11, 12], a more gentle division, introduced in refs. [26, 27] and inspired by the Catani-Seymour work [24], is exploited:

$$
\frac{d \alpha_{1}}{\alpha_{1}} \frac{d \beta_{1}}{\beta_{1}}\left(m_{F}+m_{B}\right)=\frac{d \alpha_{1} d \beta_{1}}{\beta_{1}\left(\alpha_{1}+\beta_{1}\right)}+\frac{d \alpha_{1} d \beta_{1}}{\alpha_{1}\left(\alpha_{1}+\beta_{1}\right)}, \quad m_{F}=\frac{\alpha_{1}}{\alpha_{1}+\beta_{1}}, m_{B}=\frac{\beta_{1}}{\alpha_{1}+\beta_{1}},
$$

\footnotetext{
${ }^{3}$ The subscript in $k_{1}$ and $s_{1}$ is kept to underline that, in the context of PSMC, there is more parton emissions further away from the hard process $q \bar{q} Z$ vertex.
} 
where $m_{F, B}$ are the so-called soft partition functions. What is important for our methodology in the following is that the sum of the two parts attributed to two emitters/showers reproduces the original 1-particle phase space without any gaps and (or well-controlled) overlaps. Clearly the $m_{F}+m_{B}=1$ property of the functions used in eq. (2.6) takes care of that.

Once the above (overlapping) separation of the emission phase space into two part is applied, a different evolution variable of PSMC in each of them is defined

$$
q_{1_{F}}^{2}=s_{0}\left(\alpha_{1}+\beta_{1}\right) \beta_{1}, \quad q_{1_{B}}^{2}=s_{0}\left(\alpha_{1}+\beta_{1}\right) \alpha_{1},
$$

instead of a common one, like $k_{T}^{2}=\alpha_{1} \beta_{1} s_{0}$ or rapidity.

Using the above evolution variable and $z_{1}$, the single-emission (eikonal) phase space (2.5) is easily re-parametrized

$$
\frac{d \alpha_{1} d \beta_{1}}{\alpha_{1} \beta_{1}}=\frac{d q_{1_{F}}^{2}}{q_{1_{F}}^{2}} \frac{d z_{1}}{1-z_{1}}+\frac{d q_{1_{B}}^{2}}{q_{1_{B}}^{2}} \frac{d z_{1}}{1-z_{1}} .
$$

The relation between the old and new variables are illustrated graphically in figure 2 . The transformation back to the Sudakov variables is different for each part:

$$
\begin{array}{ll}
\beta_{1}=\frac{\left(q_{1_{F}}^{2} / s_{0}\right)}{1-z_{1}}, & \alpha_{1}=1-z_{1}-\frac{\left(q_{1_{F}}^{2} / s_{0}\right)}{1-z_{1}}, \\
\alpha_{1}=\frac{\left(q_{1_{B}}^{2} / s_{0}\right)}{1-z_{1}}, & \beta_{1}=1-z_{1}-\frac{\left(q_{1_{B}}^{2} / s_{0}\right)}{1-z_{1}} .
\end{array}
$$

The upper phase-space limit $\alpha_{1}+\beta_{1} \leq 1$ transforms into

$$
z_{1} \geq 0 \quad \text { and } \quad q_{1_{F}, 1_{B}}^{2} \leq s_{0},
$$

while the positivity conditions, $\alpha_{1}>0$ and $\beta_{1}>0$, enforce the IR-boundary cut-offs

$$
\left(1-z_{1}\right)^{2}>\frac{q_{1_{F}}^{2}}{s_{0}} \quad \text { or } \quad\left(1-z_{1}\right)^{2}>\frac{q_{1_{B}}^{2}}{s_{0}}
$$

for the two parts, correspondingly. Also, in most of the phase space region populated according to the $\sim m_{F}$ factor we may approximate $q_{1_{F}}^{2} \simeq k_{1 T}^{2}$. Similarly $q_{1_{B}}^{2} \simeq k_{1 T}^{2}$ in the $\sim m_{B}$ phase-space sector. The above kinematical limits are also shown on the logarithmic Sudakov plane in figure 2 for the $\sim m_{F}$ sector. NB. The IR cut-off $k_{1 T}^{2}>k_{T \text { min }}^{2}$ marked in this figure translates into a slightly stronger cut-off on $1-z_{1}$, easily calculable. The same kinematical limits for one emission are also illustrated directly in terms of the $1-z_{1}$ and $q_{1_{F}}^{2}$ variables in figure 3 , including also the second emission for the purpose of the following discussion.

The essential ingredient of the 1-particle phase space reorganization towards PSMC is introduction of the on-shell "effective beams" $p_{1_{F}}$ and $p_{1_{B}}$, such that $p_{1_{F}}+p_{1_{B}}=$ $p_{0_{F}}+p_{0_{B}}-k_{1}$. Their definition is not unique. For example, for the $\sim m_{F}$ branch one may choose

$$
p_{1_{B}}=(1-\epsilon) p_{0_{B}}, \quad p_{1_{F}}=p_{0_{F}}-k_{1}+\epsilon p_{0_{B}}, \quad \epsilon=\beta_{1} /\left(1-\alpha_{1}\right)
$$




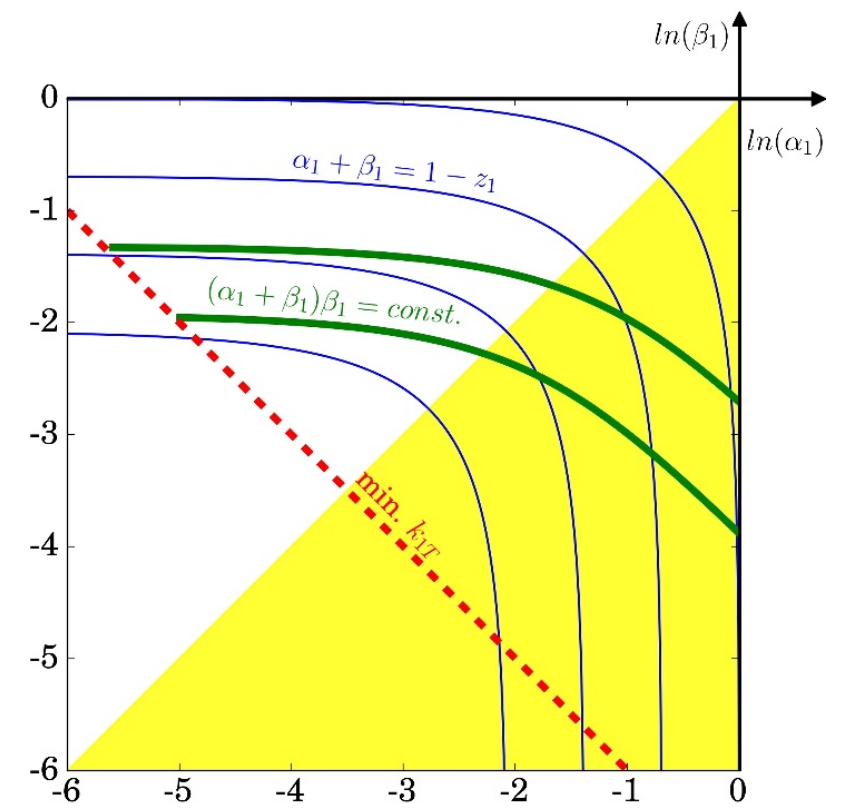

Figure 2. The Sudakov logarithmic plane with lines marking $q_{1_{F}}^{2}=$ const. (thick green) and $z_{1}=$ const. (thin blue). The dashed (red) line marks IR cut-off on $k_{1 T}=$ const.. The shaded (yellow color) triangle marks the part of the phase space where $\sim m_{F}$ dominates.

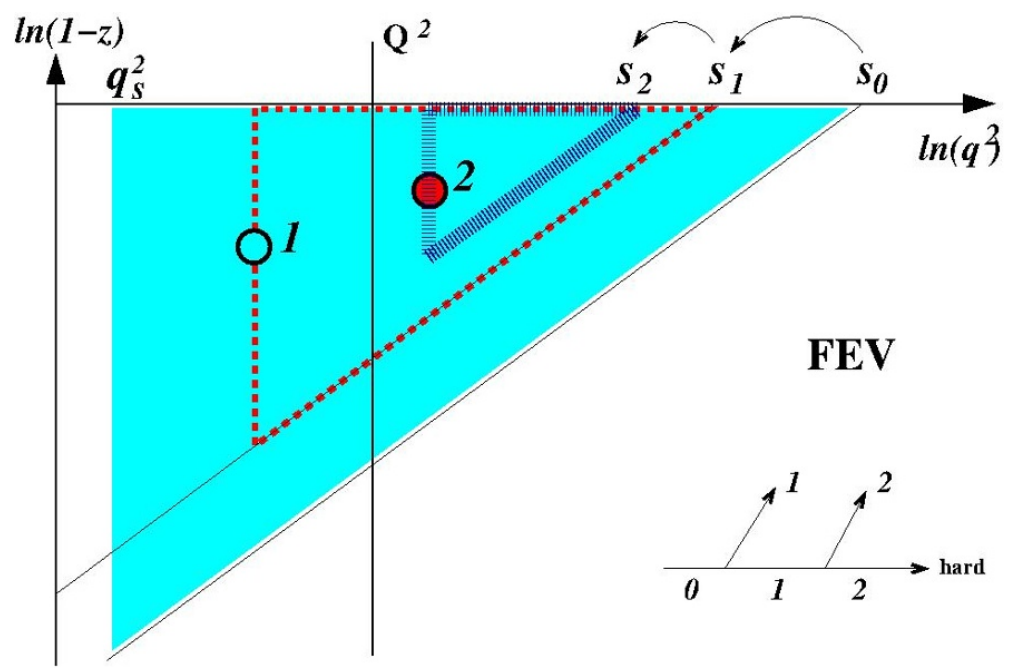

Figure 3. The illustration of the kinematic boundaries in the forward evolution (FEV) algorithm with $n=2$ particles. The parton no. 1 is emitted as a first one, within the biggest shaded triangular (blue) area defined by $\left(1-z_{1}\right)^{2}>q_{1}^{2} / s_{0}$ and $q_{1}^{2}>q_{s}^{2}$. The parton no. 2 is generated as a smaller triangular area marked with dashed (red) line according to $\left(1-z_{2}\right)^{2}>q_{2}^{2} / s_{1}$ and $q_{2}^{2}>q_{1}^{2}$. Third parton is not generated, but its would-be-allowed space is marked by the smallest triangle below $s_{2}=\hat{s}$. The vertical line marking the upper phase-space boundary $q^{2}<Q^{2}$ in $\bar{D}\left(Q^{2}, x\right)$ of eq. (3.24) is also marked. 
The same for the $\sim m_{B}$ branch modulo the obvious variables interchange. All that in the rest frame of $P_{0}=p_{0_{F}}+p_{0_{B}}$. However, in practice of a typical PSMC with the backward evolution, all four-momenta are reconstructed starting from $q_{1_{F}, 1_{B}}^{2}$ and $z_{1}$ variables in the rest frame of the effective beams $P_{1}=p_{1_{F}}+p_{1_{B}}$, which are constructed in the first place.

Technical details of the construction of effective beams are not so important for our analysis. Just as an illustration let us define explicitly one possible construction, which was introduced in ref. [23]. In the rest frame of the hard process and the effective beams $P_{1}=p_{1_{F}}+p_{1_{B}}=(\sqrt{\hat{s}}, 0,0,0)$ one may construct all four-momenta - starting from the $\left(q_{1_{F}}, z_{1}, \phi_{1}\right)$ set, then translating it into $\left(\alpha_{1}, \beta_{1}\right)$ and using (for $\left.\sim m_{F}\right)$ :

$$
\begin{aligned}
k_{1}^{\mu} & =\alpha_{1}^{*} p_{1_{F}}^{\mu}+\beta_{1}^{*} p_{1_{B}}^{\mu}+q_{T, 1}^{\mu}, & q_{T, 1}^{2} & =\alpha_{1}^{*} \beta_{1}^{*} \hat{s}, \\
p_{0_{B}}^{\mu} & =\frac{\beta_{1}+z_{1}}{z_{1}} p_{1_{B}}^{\mu}, & p_{0_{F}}^{\mu} & =P_{1}^{\mu}+k_{1}^{\mu}-p_{0_{B}}^{\mu}, \\
\alpha_{1}^{*} & =\frac{1-\beta_{1}-z_{1}}{\beta_{1}+z_{1}}, & \beta_{1}^{*} & =\frac{1}{\beta_{1}+z_{1}} \frac{\beta_{1}}{z_{1}} .
\end{aligned}
$$

Finally, knowing $P_{0}=P_{1}-k_{1}$ one may transform all newly constructed four-momenta $k_{1}, p_{0_{F}}, p_{0_{B}}$ to the rest frame of $P_{0}$.

Altogether, the complete reorganization of the 1-real emission phase space from a simple form based on the Sudakov variables to an equivalent parametrization using the variables of PSMC, based on the backward evolution algorithm (applying the CataniSeymour soft-partition factor $m_{F}$ ), keeping track of the kinematical limits, and defining $\hat{x}=x_{1}=x_{0} z_{1}, \hat{s}=s_{1}=s x_{1}$, looks as follows:

$$
\begin{aligned}
d \sigma_{1_{F}} & \simeq \int_{0}^{1} d x_{1} D\left(\mu_{F}^{2}, x_{1}\right) \int_{\alpha_{1}+\beta_{1} \leq 1} \frac{d \beta_{1} d \alpha_{1}}{\beta_{1}\left(\alpha_{1}+\beta_{1}\right)} \bar{P}\left(z_{1}\right) d \sigma_{0}\left(s x_{0} z_{1}\right) \\
& =\int_{0}^{1} d x_{1} \int_{q_{\min }^{2}}^{s_{0}} \frac{d q_{1}^{2}}{q_{1}^{2}} \int_{0}^{1-\sqrt{q_{1}^{2} / s_{0}}} d z_{1} \frac{\bar{P}\left(z_{1}\right)}{1-z_{1}} d \sigma_{0}\left(s x_{1}\right) D\left(\mu_{F}^{2}, x_{1}\right) \\
& =\int_{0}^{1} d \hat{x} \int_{q_{\min }^{2}}^{s} \frac{d q_{1}^{2}}{q_{1}^{2}} \int_{x_{0}}^{1} d z_{1} \theta_{\left(1-z_{1}\right)^{2} s \hat{x} / z_{1} \geq q_{1}^{2}} P_{q \bar{q}}\left(z_{1}\right) d \sigma_{0}(s \hat{x}) D\left(\mu_{F}^{2}, \frac{\hat{x}}{z_{1}}\right),
\end{aligned}
$$

where $q_{1}^{2}=q_{1_{F}}^{2}, \bar{P}(z) \equiv(1-z) P_{q \bar{q}}(z)$ and $P_{q \bar{q}}(z)$ is the DGLAP [28] splitting function. It is important to see that the full phase coverage requires integration over $q_{F}^{2}$ to extend above the effective mass squared $\hat{s}=s_{2}$ of the LO hard process. Since $q_{F}^{2} \simeq k_{1 T}^{2}$, it means that the transverse momentum above $\hat{s}$ is included in the above phase space.

In section 5 presenting numerical results, it will be commented more on what kind of evolution variable is chosen in the parton shower generation of Sherpa and Herwig ++ .

\section{$2.2 \quad$ Multiple emissions}

Although the above 1-emission kinematics is enough for most of our prescription for the NLO-correcting of the hard process, for better understanding of the role of the underlying 


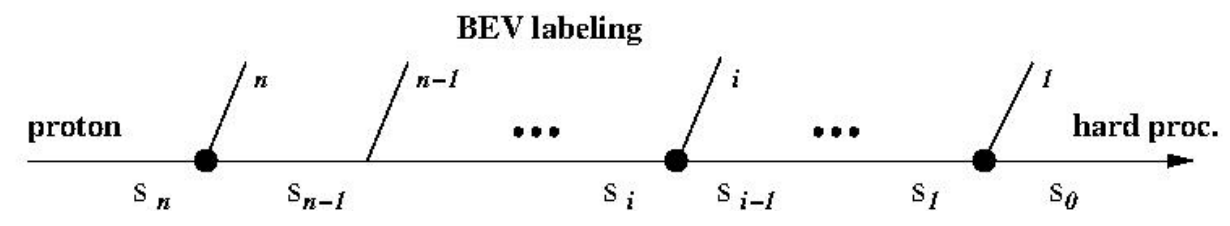

Figure 4. Labelling in the reconstruction of the four-momenta.

multi-emission PSMC, it is useful to extend the 1-emission treatment of the kinematics to two and more emissions in the initial-state parton showers.

The important technical point is the choice of the numbering (labelling) of the emitted particles. In figure 3 we use the numbering in the emission chain (ladder) starting at the incoming hadron and ending next to the hard process, that is the labelling of the forward evolution (FEV) algorithm. From now on we switch to the backward evolution (BEV) labelling which starts next to the hard process and ends at the incoming hadron, see figure 4 for illustration.

Let us summarize briefly on the effective beam technique, for simplicity limiting its description to a single tree $F$ of emissions (shower). The sequence of the effective emitter beams $\left(\tilde{p}_{i_{F}}, \tilde{p}_{i_{B}}\right)$ is defined starting from the hard process, with the four-momentum $P_{i}$, such that

$$
P_{i}=\tilde{p}_{i_{F}}+\tilde{p}_{i_{B}}, \quad P_{i+1}=P_{i}+k_{i}, \quad P_{i}^{2}=\hat{s} /\left(\prod_{j=1}^{i} z_{i}\right),
$$

and they are used to span the four-momentum of the emitted gluon

$$
k_{i}=\tilde{\alpha}_{i} \tilde{p}_{i_{F}}+\tilde{\beta}_{i} \tilde{p}_{i_{B}}+k_{T i}, \quad \tilde{\alpha}_{i}=\frac{k_{i} \tilde{p}_{i_{B}}}{\tilde{p}_{i_{F}} \tilde{p}_{i_{B}}}, \quad \tilde{\beta}_{i}=\frac{k_{i} \tilde{p}_{i_{F}}}{\tilde{p}_{i_{F}} \tilde{p}_{i_{B}}}
$$

introducing the Sudakov variables $\tilde{\alpha}_{i}, \tilde{\beta}_{i}$. These Sudakov variables are related to the evolution variable $q_{i_{F}}^{2}$ and the light-cone variable $z_{i}$ of PSMC as follows:

$$
\tilde{\beta}_{i}=\frac{q_{i}^{2} Z_{i}}{\hat{s}\left(1-z_{i}\right)}, \quad \tilde{\alpha}_{i}=1-z_{i}-\tilde{\beta}_{i}, \quad Z_{i}=\prod_{j=1}^{i} z_{j}, \quad \hat{s}=s \hat{x}=s x_{0} .
$$

Finally, in the recursive backward reconstruction of the four-momenta starting from the hard process, one employs the Sudakov-like decomposition of the emitted parton in terms of the emitters after the emission: ${ }^{4}$

$$
\begin{aligned}
& k_{i}^{\mu}=\alpha_{i}^{*} \tilde{p}_{(i-1)_{F}}^{\mu}+\beta_{i}^{*} \tilde{p}_{(i-1)_{B}}^{\mu}+q_{T, i}^{\mu}, \\
& \alpha_{i}^{*}=\frac{\tilde{\alpha}_{i}}{1-\tilde{\alpha}_{i}}, \quad \beta_{i}^{*}=\frac{1}{1-\tilde{\alpha}_{i}} \frac{\tilde{\beta}_{i}}{z_{i}} .
\end{aligned}
$$

All exact kinematical limits (including ordering in the evolution variable) are represented by the following inequalities:

$$
\left(1-z_{i}\right)^{2} \geq q_{i}^{2} Z_{i} / \hat{s} \quad \text { and } \quad q_{i+1}^{2} \leq q_{i}^{2} \leq \hat{s}\left(1-z_{i}\right)^{2} / Z_{i}
$$

\footnotetext{
${ }^{4}$ Generalizing eq. (2.13).
} 


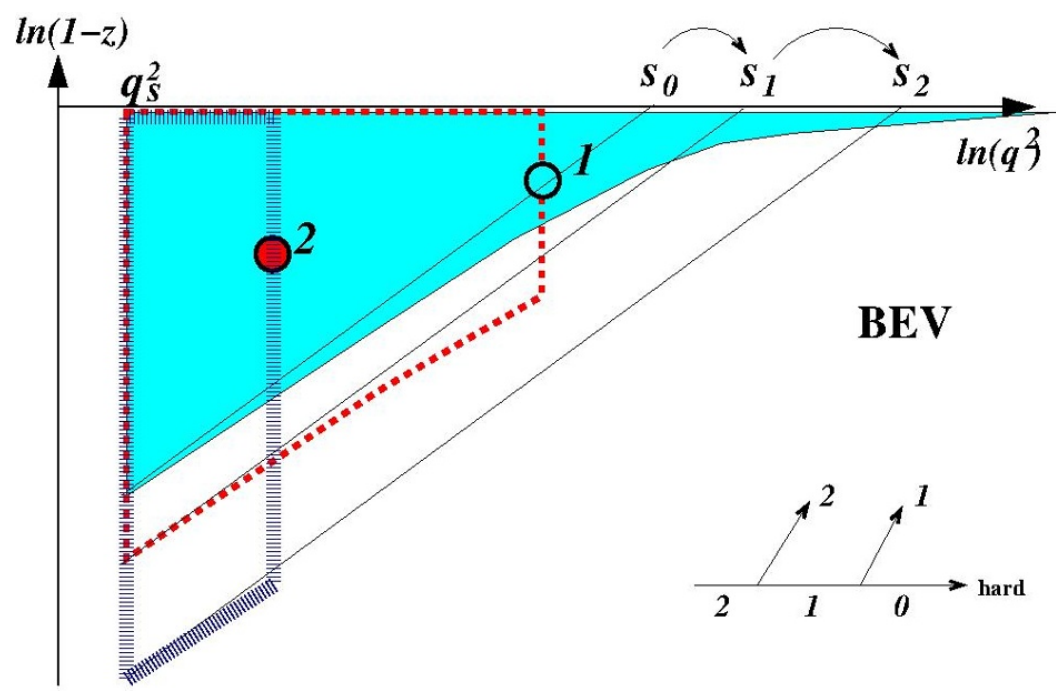

Figure 5. The illustration of the kinematic boundaries in the BEV algorithm with $n=2$ particles. The parton no. 1 is emitted within the semi-triangular shaded (blue) area defined by $\left(1-z_{1}\right)^{2}>q_{1}^{2} /\left(s_{0} / z_{1}\right)$ and $s>q_{1}^{2}>q_{s}^{2}$. The parton no. 2 is generated within the second semitrapezoid area marked by the dashed (red) line according to $\left(1-z_{2}\right)^{2}>q_{2}^{2} /\left(s_{1} / z_{2}\right)$ and $q_{1}^{2}>q_{2}^{2}>q_{s}^{2}$. The third parton is not generated, but its would-be-allowed space is marked by the leftmost almosttrapezoid line.

In particular, the kinematical limits for the first emission in the backward-evolution (BEV) labelling are

$$
q_{\max , 1}^{2}=\hat{s}\left(1-z_{1}\right)^{2} / z_{1}, \quad z_{1}^{\max } \simeq 1-q_{0} / \sqrt{\hat{s}} .
$$

The above kinematical limits in terms of the BEV variables $q_{i}^{2}$ and $z_{i}$ look more complicated than in the FEV scenario, although they represent exactly the same phase space region, and are illustrated graphically in figure 5 .

As already underlined, in terms of the BEV variables, the phase space gets apparently widened after each emission, for instance $q^{2}>\hat{s}$ is already available for the first emission and, due to lowering of the IR boundary by the $1 / z_{1}$ factor, more phase space is available for the second emission. This phenomenon, important for the full coverage of the phase space, is illustrated graphically in figure 6. It is, of course, an artefact of the BEV phasespace parametrization, which in the FEV world corresponds to the phase-space reduction due to energy conservation.

We omit here the discussion of the "kinematical cross-talk" between two parton showers, which means that for emissions with the common $q^{2}$-ordering in two initial showers (as in any realistic PSMC), the emission in one shower reduces the available phase space in the other shower. This effect is easily incorporated in the kinematical construction (mappings) of PSMC. The only thing one has to watch out is the correctness of the soft-emission limit in the case of two and more emissions, see for instance the discussion in ref. [23]. This subject will be covered in a more detail in our future publications. 


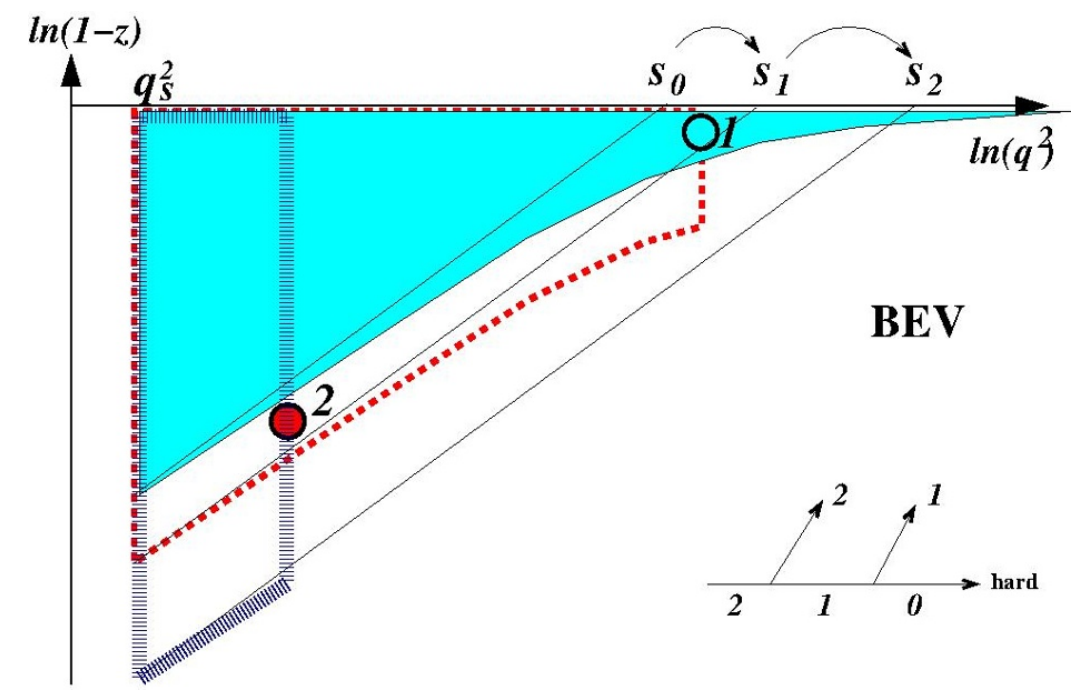

Figure 6. The illustration of the kinematic of the BEV algorithm with $n=2$ particles in the extreme case when the parton no. 1 is emitted with $q_{1}^{2}>s_{0}=\hat{s}$ and the parton no. 2 is generated within the area not accessible for the parton no. 1 due to a higher IR boundary.

\section{KrkNLO methodology}

Very briefly, the essence of the KrkNLO prescription defined in ref. [19] is that NLO corrections are introduced by a multiplicative weight on top of distributions from the LO PS acting, either inside PSMC or outside it, on a MC event record. This NLO weight is sensitive to the parton four-momentum with the highest $q^{2}$ (or maximum $k_{T}^{2}$ in the case of $k_{T}$-ordering), although in ref. [19] it was demonstrated that such a NLO multiplicative weight works also in the case of the angular ordering, provided that summation over all emitted partons is performed.

In MC@NLO [2], the correcting weight is essentially additive and the NLO - LO correction to PSMC is provided from outside in form of additional MC events, with a nonpositive-definite weight. In parts of the phase space which are not covered by the LO PS, extra events provide the entire NLO distributions (positive weights), otherwise extra events, correcting the LO distributions to the NLO level, have typically (inconvenient) negative weights.

In POWHEG [3], the entire NLO correction to LO PS is provided by an external MC generator - the highest $k_{T}^{2}$ emission is isolated/subtracted from PSMC, following the double-logarithmic Sudakov exponential factor, and generated according to the NLO distribution outside PSMC, while trailing emissions with lower $k_{T}^{2}$ (suppressed by the Sudakov exponent) are left for generating within LO PSMC.

Both KrkNLO and MC@NLO require good analytical control of the LO PS distributions, while for POWHEG it is less important. In addition, KrkNLO requires that LO PS fills in the entire NLO phase space with some non-zero distribution. The Sudakov suppression is also exploited in KrkNLO, but in a different way than in POWHEG. 
In the standard NLO corrections to a hard process with $\overline{\mathrm{MS}}$ PDFs, part of the NLO corrections feature degenerate 1-dimensional longitudinal phase space with $k_{T}^{2}=0$ exactly. These corrections enforce in practice certain convolution on the $\overline{\mathrm{MS}} \mathrm{PDFs}$, which in the case of POWHEG and MCONLO is done in-flight inside MC. In the KrkNLO prescription, the implementation of these corrections is moved outside MC (simplifying it). This leads to the use in KrkNLO of the modified LO PDFs, in the so-called MC factorization scheme. This reorganization is mandatory because one of the main aims of the KrkNLO method, that the NLO corrections are implemented with a well-behaved multiplicative positive weight, is not compatible with such a degenerate collinear phase-space contribution to the NLO MC distributions.

Last but not least, any scheme for correcting the hard process of LO PSMC to the NLO level requires a formal proof that the resulting distributions are indeed of the NLO class (no double-counting, no NLO leak). Such a proof in an algebraic form is not trivial, not only because the NLO total cross section has to be verified, but also any NLO-class observable (experimental event selections) has to be properly reproduced. In other words, it has to be done using functional space of all the NLO observables. In the case of KrkNLO such a proof was done in ref. [19], both algebraically and numerically, for a toy-model PSMC with the angular ordering. Here, we shall provide such an algebraic proof starting from the NLO-corrected multiparton distributions for realistic PSMC of the kind implemented in Sherpa and Herwig++ using the BEV algorithm.

In the following, we are going to collect building blocks for the NLO weight, then we shall elaborate on the multiparton distributions of LO PSMC without and with the NLO weight of KrkNLO. We shall pay particular attention to the question of the completeness of the phase space in PSMC and to the equivalence between the backward and forward evolution algorithms in PSMC. Finally, we shall show that for an arbitrary NLO-class observable, KrkNLO gives the same result as simpler NLO calculations with the collinear PDFs, instead of PSMC, such as for instance MCFM [32].

\subsection{NLO-correcting weight}

Let us collect the ingredients for construction of the NLO corrections to the hard process of the $Z$-boson production and decay in the proton-proton collisions.

The fully differential NLO cross section of the production and decay of the $Z$ boson in the quark-antiquark annihilation process, with the simultaneous emission of a single real gluon, can be cast in a well-known compact form, see ref. [19]:

$$
d^{5} \sigma_{q \bar{q}}^{N L O}(\alpha, \beta, \Omega)=\frac{C_{F} \alpha_{s}}{\pi} \frac{d \alpha d \beta}{\alpha \beta} \frac{d \varphi}{2 \pi} d \Omega\left[\frac{d \sigma_{0}\left(\hat{s}, \theta_{F}\right)}{d \Omega} \frac{(1-\beta)^{2}}{2}+\frac{d \sigma_{0}\left(\hat{s}, \theta_{B}\right)}{d \Omega} \frac{(1-\alpha)^{2}}{2}\right],
$$

where the Sudakov variables ${ }^{5}(\alpha, \beta)$ are spanned on momenta of the effective beams of $q$ and $\bar{q}$ prior to the gluon emission, see eq. (2.4). The Born differential cross section $\frac{d \sigma_{0}\left(\hat{s}, \theta_{B}\right)}{d \Omega}$ for $Z$-boson production and decay is well known (see for instance [25] for the exact expression).

\footnotetext{
${ }^{5}$ For better readability of the formulae given in this section, we shall suppress "1" in the lower index of the Sudakov variables as well as other kinematical variables, such as $s$ and $q_{F, B}^{2}$.
} 
The solid angle $\Omega=(\theta, \phi)$ is the direction of the lepton from the decaying $Z$ boson in its rest frame and $\hat{s}=(1-\alpha-\beta) s_{1}$ (see figure 4). The angles $\theta_{F}$ and $\theta_{B}$ depend on $\alpha$ and $\beta$ as well - their precise definition was given ${ }^{6}$ in ref. [33]. The integration over luminosities of $q$ and $\bar{q}$ is not yet included.

How does the above compare with the distributions from PSMC also restricted to the single gluon emission? The differential cross section for the gluon emission from the quark emitter (i.e. the $\sim m_{F}$ part in eq. (2.6)) in PSMC reads

$$
\begin{aligned}
d^{5} \sigma_{q \bar{q}}^{F}(\alpha, \beta, \Omega) & =\frac{C_{F} \alpha_{s}}{\pi} \frac{d q_{F}^{2}}{q_{F}^{2}} \frac{d \varphi}{2 \pi} P_{q \bar{q}}(z) d z \frac{d \sigma_{0}}{d \Omega}(\hat{s}, \hat{\theta}) d \Omega \\
& =\frac{C_{F} \alpha_{s}}{\pi} \frac{d \alpha d \beta}{(\alpha+\beta) \beta} \frac{d \varphi}{2 \pi} d \Omega \frac{1+(1-\alpha-\beta)^{2}}{2} \frac{d \sigma_{0}}{d \Omega}(\hat{s}, \hat{\theta})
\end{aligned}
$$

where $q_{F}$ is the evolution variable defined in eq. (2.7) and $\hat{\theta}$ is another effective angle in $Z$ decay specific to LO PSMC, for instance the so-called Collins-Soper angle [34]. Adding the gluon emission from $\bar{q}$ simply amounts to the $\alpha \leftrightarrow \beta$ symmetrization, resulting in

$$
d^{5} \sigma_{q \bar{q}}^{\mathrm{LO}}(\alpha, \beta, \Omega)=d^{5} \sigma_{q \bar{q}}^{F}+d^{5} \sigma_{q \bar{q}}^{B}=\frac{C_{F} \alpha_{s}}{\pi} \frac{d \alpha d \beta}{\alpha \beta} \frac{d \varphi}{2 \pi} d \Omega \frac{1+(1-\alpha-\beta)^{2}}{2} \frac{d \sigma_{0}}{d \Omega}(\hat{s}, \hat{\theta}),
$$

where

$$
d^{5} \sigma_{q \bar{q}}^{F}=\frac{\alpha}{\alpha+\beta} d^{5} \sigma_{q \bar{q}}^{\mathrm{LO}}=m_{F} d^{5} \sigma_{q \bar{q}}^{\mathrm{LO}}, \quad d^{5} \sigma_{q \bar{q}}^{B}=\frac{\beta}{\alpha+\beta} d^{5} \sigma_{q \bar{q}}^{\mathrm{LO}}=m_{B} d^{5} \sigma_{q \bar{q}}^{\mathrm{LO}} .
$$

The integration limits are not explicit, but they are the same as in eq. (2.4).

In our discussion, we shall often use the following objects: the additive NLO correction

$$
d^{5} \bar{\beta}_{q \bar{q}}(\alpha, \beta, \Omega)=d^{5} \sigma_{q \bar{q}}^{\mathrm{NLO}}(\alpha, \beta, \Omega)-d^{5} \sigma_{q \bar{q}}^{\mathrm{LO}}(\alpha, \beta, \Omega)
$$

and the NLO multiplicative weight for the $q \bar{q}$ channel

$$
W_{q \bar{q}}^{(1)}(\alpha, \beta)=1+\frac{d^{5} \bar{\beta}_{q \bar{q}}}{d^{5} \sigma_{q \bar{q}}^{\mathrm{LO}}}=\frac{d^{5} \sigma_{q \bar{q}}^{\mathrm{NLO}}}{d^{5} \sigma_{q \bar{q}}^{\mathrm{LO}}} .
$$

The above weight is especially simple in the case of averaging over the angles in $Z$ decay:

$$
\left\langle W_{q \bar{q}}^{(1)}\right\rangle_{\Omega}=W_{R}^{q \bar{q}}=1-\frac{2 \alpha \beta}{1+(1-\alpha-\beta)^{2}},
$$

and it can be used in approximate implementations of the NLO corrections.

The analogous weight for the $q g$ channel is

$$
\left\langle W_{q g}^{(1)}\right\rangle_{\Omega}=W_{R}^{q g}=1+\frac{\beta(\beta+2 z)}{(1-z)^{2}+z^{2}} .
$$

After summing up the contributions from the two emitters, $d^{5} \sigma_{q \bar{q}}^{\mathrm{LO}}$ is obtained, which is exactly the same ${ }^{7}$ as in ref. [19]. The important consequence of the above is that many

\footnotetext{
${ }^{6}$ In fact they coincide with the polar angles with respect to the effective beams defined in eq. (2.12).

${ }^{7}$ In spite of the differences of the LO PSMC distributions for each emitter separately.
} 
details of the matching between the $\overline{\mathrm{MS}}$ NLO corrections and the LO PSMC in the present KrkNLO implementation remains the same as in ref. [19]. In particular, the virtual plus soft-real correction, when the PDFs in the MC factorization scheme are used, is the same as in ref. [19] and reads ${ }^{8}$

$$
\bar{B}_{\mathrm{MC}}^{i j}=1+\Delta_{V S}^{i j}
$$

where

$$
\Delta_{V S}^{q \bar{q}}=\frac{\alpha_{s}}{2 \pi} C_{F}\left[\frac{4}{3} \pi^{2}-\frac{5}{2}\right], \quad \quad \Delta_{V S}^{q g}=0 .
$$

As one can see, this virtual+soft-real correction is constant, i.e. kinematics-independent.

\subsection{MC factorization scheme}

In this subsection we extend the definition of the MC factorization scheme, introduced in ref. [19] for the quark-antiquark channel, to the NLO DY process with the quark-gluon initial state. For the completeness and convenience of the reader we first provide the main formulae for the $q \bar{q}$ initial state.

The NLO $q \bar{q}$-channel coefficient function for the DY process in the $\overline{\text { MS }}$ factorization scheme is given by [19]

$$
C_{2 q}^{\overline{\mathrm{MS}}}(z)=\frac{\alpha_{s}}{2 \pi} C_{F}\left\{\delta(1-z)\left(\frac{4}{3} \pi^{2}-\frac{7}{2}\right)+\left[2 \frac{1+z^{2}}{1-z} \ln \frac{(1-z)^{2}}{z}\right]_{+}\right\} .
$$

The corresponding coefficient function in the MC factorization scheme, defined in ref. [19], reads

$$
C_{2 q}^{\mathrm{MC}}(z)=\frac{\alpha_{s}}{2 \pi} C_{F}\left[\delta(1-z)\left(\frac{4}{3} \pi^{2}-\frac{7}{2}\right)-2(1-z)_{+}\right]=\delta(1-z) \Delta_{V S}-\frac{\alpha_{s}}{\pi} C_{F}(1-z),
$$

where $\Delta_{V S} \equiv \Delta_{V S}^{q \bar{q}}$ is the virtual plus soft-real gluonstrahlung correction given in eq. (3.10).

From the above equations, following ref. [19], we can obtain a $q \bar{q}$ contribution to the relation between the MC-scheme and $\overline{\mathrm{MS}}$-scheme quark (antiquark) PDFs:

$$
\Delta C_{2 q}(z)=\frac{1}{2}\left[C_{2 q}^{\overline{\mathrm{MS}}}(z)-C_{2 q}^{\mathrm{MC}}(z)\right]=\frac{\alpha_{s}}{2 \pi} C_{F}\left[\frac{1+z^{2}}{1-z} \ln \frac{(1-z)^{2}}{z}+1-z\right]_{+} .
$$

Similarly, for the NLO $q g$-channel contribution to the DY process we have:

$$
\begin{aligned}
C_{2 g}^{\overline{\mathrm{MS}}}(z) & =\frac{\alpha_{s}}{2 \pi} T_{R}\left\{\left[z^{2}+(1-z)^{2}\right] \ln \frac{(1-z)^{2}}{z}-\frac{7}{2} z^{2}+3 z+\frac{1}{2}\right\}, \\
C_{2 g}^{\mathrm{MC}}(z) & =\frac{\alpha_{s}}{2 \pi} T_{R} \frac{1}{2}(1-z)(1+3 z), \\
\Delta C_{2 g}(z) & =C_{2 g}^{\overline{\mathrm{MS}}}(z)-C_{2 g}^{\mathrm{MC}}(z)= \\
& =\frac{\alpha_{s}}{2 \pi} T_{R}\left\{\left[z^{2}+(1-z)^{2}\right] \ln \frac{(1-z)^{2}}{z}+2 z(1-z)\right\} .
\end{aligned}
$$

\footnotetext{
${ }^{8}$ Note that $\bar{B}_{\mathrm{MC}}^{i j}$, though conceptually similar, is not identical to the well known $\bar{B}$ used in the context of POWHEG [35], as the quantity from eq. (3.9) is defined in the MC scheme.
} 
Using eqs. (3.13) and (3.16), we can relate the MC-scheme quark (antiquark) PDFs to the corresponding $\overline{\mathrm{MS}}$-scheme PDFs in the following way

$$
f_{q(\bar{q})}^{\mathrm{MC}}\left(x, Q^{2}\right)=f_{q(\bar{q})}^{\overline{\mathrm{MS}}}\left(x, Q^{2}\right)+\int_{x}^{1} \frac{d z}{z} f_{q(\bar{q})}^{\overline{\mathrm{MS}}}\left(\frac{x}{z}, Q^{2}\right) \Delta C_{2 q}(z)+\int_{x}^{1} \frac{d z}{z} f_{g}^{\overline{\mathrm{MS}}}\left(\frac{x}{z}, Q^{2}\right) \Delta C_{2 g}(z) .
$$

The above relation is universal, i.e. process-independent, at the NLO level. It is simply because it is defined uniquely with respect to the $\overline{\mathrm{MS}}$ scheme.

The gluon PDF is equal between the MC and $\overline{\mathrm{MS}}$ schemes up to the $\mathcal{O}\left(\alpha_{s}{ }^{2}\right)$ corrections for processes with no gluons at the Born level, such as the Drell-Yan process considered in this work. Hence for the DY process we may use

$$
f_{g}^{\mathrm{MC}}\left(x, Q^{2}\right)=f_{g}^{\overline{\mathrm{MS}}}\left(x, Q^{2}\right)
$$

As one can see, in the MC factorization scheme the NLO coefficient functions in both the $q \bar{q}$ and $q g$ channels are substantially simpler than the corresponding ones in the $\overline{\mathrm{MS}}$ scheme; in particular they are free of logarithmic singular terms. Since the latter terms are absorbed into the MC PDFs, i.e. they are included in a resummed way, one may also expect that the higher-order QCD corrections in the MC scheme are smaller than in the standard $\overline{\mathrm{MS}}$ scheme.

Let us finally add a comment on the universality (process-independence) of the MC factorization scheme. This issue was discussed quite extensively in ref. [19] for the DIS and DY processes, albeit restricting the problem to QED-like gluonstrahlung diagrams only. The main point is that the MC factorization scheme is in reality defined operationally as a modification of the $\overline{\mathrm{MS}}$ scheme, consequently it inherits automatically the universality from the latter. ${ }^{9}$ On the other hand, it is true that the procedure of defining extra $\mathcal{O}\left(\epsilon^{0}\right)$ terms, added to the $\overline{\mathrm{MS}}$ collinear counter-terms to define the PDFs in the MC scheme, is clearly guided by the inspection of a number of simplest physical processes, like DIS, DY and/or Higgs production. However, once this is done, these extra terms are frozen and the resulting (modified) counter-terms are ready to apply for any other process. They also define completely the PDFs in the MC scheme. What is new in the present work with respect to ref. [19] is the inclusion of the quark-gluon transitions. Generally, the transformation of the PDFs from the $\overline{\mathrm{MS}}$ to the MC scheme is a matrix in the flavor space. The DY process at the NLO level, discussed in the present work, fixes only a subset of terms in this matrix, while the remaining ones will be fixed by inspecting the NLO corrections to the process of gluon-gluon fusion into the Higgs boson. ${ }^{10}$ At the next step, after including Higgs production in the game, the MC scheme will be fully defined and will be applicable to any process, including also more color particles in the final state.

\footnotetext{
${ }^{9}$ In particular, the MC scheme is neither the DIS nor the DY scheme, as can be seen from the fact that the coefficient functions for any of these processes in the MC scheme are not equal to $\delta(1-z)$.

${ }^{10}$ They are set temporarily to zero in the present work, as for the DY process they become relevant only at NNLO.
} 


\subsection{Multiparton reality of PSMC}

In the above we have restricted ourselves to a single emission and hence omitted all the multi-emission reality of PSMC. Let us elaborate on that in a more detail now, because the NLO correcting weights are put not on top of single-emission distributions but on top of multiparton distributions of LO PSMC, so they have to be known and controlled for the hard process within the NLO precision, preferably in a closed algebraic form.

Let us start from LO PSMC in the forward-evolution (FEV) formulation. The equivalent backward-evolution (BEV) formulation will be presented later on. Restricting ourselves temporarily to the pure gluonstrahlung case, the FEV differential cross sections of gluons emitted from the $q$ and $\bar{q}$ emitters ${ }^{11}$ reads as follows

$$
\begin{aligned}
\sigma_{\mathrm{MC}}^{\mathrm{LO}} & =\int d x_{F} d x_{B} d \Omega \sum_{n_{F}=0}^{\infty} \sum_{n_{B}=0}^{\infty} \int d \sigma_{n_{F} n_{B}}^{\mathrm{LO}}, \\
d \sigma_{n_{F} n_{B}}^{\mathrm{LO}} & =\prod_{i=1}^{n_{F}} \prod_{j=1}^{n_{B}}\left\{\int d^{3} \rho_{i}^{F} \theta_{q_{i-1}^{2}>q_{i}^{2}>q_{s}^{2}} e^{-S_{F}\left(q_{i-1}^{2}, q_{i}^{2}\right)}\right\}\left\{\int d^{3} \rho_{j}^{B} \theta_{q_{j-1}^{2}>q_{j}^{2}>q_{s}^{2}} e^{-S_{B}\left(q_{j-1}^{2}, q_{j}^{2}\right)}\right\} \\
& \times e^{-S_{F}\left(q_{n_{F}}^{2}, q_{s}^{2}\right)} e^{-S_{B}\left(q_{n_{B}}^{2}, q_{s}^{2}\right)} \frac{d \sigma}{d \Omega}\left(s x_{F} x_{B}, \hat{\theta}\right) \frac{1}{Z_{n_{F}}} D_{\mathrm{MC}}^{F}\left(q_{s}^{2}, \frac{x_{F}}{Z_{n_{F}}^{F}}\right) \frac{1}{Z_{n_{B}}} D_{\mathrm{MC}}^{B}\left(q_{s}^{2}, \frac{x_{B}}{Z_{n_{B}}^{B}}\right),
\end{aligned}
$$

where $Z_{i}=\prod_{l=1}^{i} z_{l}, Z_{0}=1, q_{0}^{2} \equiv s$ and $\overline{\mathcal{P}}(z)=(1-z) \mathcal{P}(z), \mathcal{P}(z) \equiv \frac{C_{F} \alpha_{s}}{\pi} P_{q \bar{q}}(z)$. The principal evolution variable $q_{i}^{2}$ was introduced in section 2 , eq. (2.7), and the labelling of the emissions starts from the hard process, ${ }^{12}$ as in figure 4 . The emission distributions for the ladder labelled with $F$ are the following:

$$
\begin{aligned}
d^{3} \rho_{i}^{F} & =d^{3} \rho_{i}^{F}\left(s_{i j}\right)=\frac{d \tilde{\beta}_{i} d \tilde{\alpha}_{i}}{\tilde{\beta}_{i}\left(\tilde{\alpha}_{i}+\tilde{\beta}_{i}\right)} \frac{d \phi_{i}}{2 \pi} \overline{\mathcal{P}}\left(1-\tilde{\alpha}_{i}-\tilde{\beta}_{i}\right) \theta_{\tilde{\alpha}_{i}>0} \theta_{\tilde{\alpha}_{i}+\tilde{\beta}_{i}<1} \\
& =\frac{d q_{i}^{2} d z_{i}}{q_{i}^{2}} \frac{d \phi_{i}}{2 \pi} \theta_{\left(1-z_{i}\right)^{2} s_{i j}>q_{i}^{2}} \mathcal{P}\left(z_{i}\right)=\frac{d q_{i}^{2}}{q_{i}^{2}} \frac{d \phi_{i}}{2 \pi} d z_{i} \frac{\overline{\mathcal{P}}\left(z_{i}\right)}{1-z_{i}} \theta_{\left(1-z_{i}\right)^{2} s_{i j}>q_{i}^{2}},
\end{aligned}
$$

where the Sudakov function reads

$$
S_{F}\left(q_{b}^{2}, q_{a}^{2}\right)=S_{F}\left(s_{i j} \mid q_{b}^{2}, q_{a}^{2}\right) \equiv \int_{q_{a}^{2}<q_{i}^{2}<q_{b}^{2}} d^{3} \rho_{i}^{F}\left(s_{i j}\right),
$$

and for the ladder ${ }_{B}$ they look the same, except for the $\tilde{\alpha}_{i} \leftrightarrow \tilde{\beta}_{i}$ swap.

The important variable $s_{i j}$ entering $d^{3} \rho_{i}^{F, B}$ and $S^{F, B}$ for the single shower/ladder was already defined in section 2 as $s_{i}=\hat{s} / Z_{i}$, with $\hat{s}=s x_{F} x_{B}$. For two showers, in any realistic PSMC, the emissions are generated (and the four-momenta are reconstructed) simultaneously in both showers using the competition algorithm, in which a common $q^{2}$ ordering in both showers is emerging in a natural way. ${ }^{13}$ Within such a common ordering

\footnotetext{
${ }^{11}$ We adopt a convention in which $\sum_{n=1}^{0} d_{n}=1$.

${ }^{12}$ This is unnatural for the present FEV scenario, but better suited for the BEV algorithm in the following.

${ }^{13}$ This method leads to forward-backward symmetric distributions, contrary to generating first the emissions from $q$ and later on all the remaining emissions from $\bar{q}$.
} 
for the two showers, the variable $s_{i j}=\hat{s} /\left(Z_{i}^{F} Z_{j}^{B}\right)$ includes all $z$ 's from the emissions in both the ladders, starting from the hard process. ${ }^{14}$

Strictly speaking, the above implicit "kinematical coupling" of the two showers through $s_{i j}$ variable prevents us from rewriting the distributions of eq. (3.19), without any approximation, into the traditional convolution of two LO PDFs and the hard process, as it was possible in the toy PS MC in refs. [19, 20]. However, a slight modification of the kinematical coupling (modulo $\mathrm{N}^{3} \mathrm{LO}$ corrections) allows us to get from eq. (3.19) the following equivalent factorized inclusive formula

$$
\sigma_{\mathrm{MC}}^{\mathrm{LO}}=\int d x_{F} d x_{B} d \Omega D_{\mathrm{MC}}^{F}\left(\hat{s}, x_{F}\right) D_{\mathrm{MC}}^{B}\left(\hat{s}, x_{B}\right) \frac{d \sigma}{d \Omega}\left(s x_{F} x_{B}, \hat{\theta}\right),
$$

where $d \sigma / d \Omega$ is the hard cross section from eq. (3.1) and the LO PDF $D_{\mathrm{MC}}^{F}$ is resulting from the FEV algorithm run separately for each single shower, written in form of the following time-ordered (T.O.) exponential ${ }^{15}$

$$
\begin{aligned}
& D_{\mathrm{MC}}^{F}\left(\hat{s}, x_{F}\right)= D_{\mathrm{MC}}^{F}\left(q_{s}^{2}, x_{F}\right) e^{-S_{F}\left(\hat{s} \mid s, q_{s}^{2}\right)}+ \\
&+\sum_{n=1}^{\infty} \int e^{-S_{F}\left(\hat{s} \mid s, q_{1}^{2}\right)} d^{3} \rho_{1}^{F}\left(s_{1}\right) e^{-S_{F}\left(s_{1} \mid q_{1}^{2}, q_{2}^{2}\right)} d^{3} \rho_{2}^{F}\left(s_{2}\right) e^{-S_{F}\left(s_{2} \mid q_{2}^{2}, q_{3}^{2}\right)} \ldots \\
& \times e^{-S_{F}\left(s_{n-1} \mid q_{n-1}^{2}, q_{n}^{2}\right)} d^{3} \rho_{n}^{F}\left(s_{n}\right) e^{-S_{F}\left(s_{n} \mid q_{n}^{2}, q_{s}^{2}\right)} \prod_{i=1}^{n} \theta_{s>q_{i-1}^{2}>q_{i}^{2}>q_{s}^{2}} D_{\mathrm{MC}}^{F}\left(q_{s}^{2}, x_{s}\right) \delta_{x_{F}=x_{s} \prod_{j=1}^{n} z_{j}},
\end{aligned}
$$

with $s_{i}=\hat{s} / Z_{i}, s_{0}=\hat{s}, q_{0}^{2} \equiv s$. The other PDF, $D_{\mathrm{MC}}^{B}\left(\hat{s}, x_{B}\right)$, is defined analogously.

It is important to note that the objects $D_{\mathrm{MC}}^{F, B}$ appearing in eq. (3.22) are not just scalar functions but they have non-trivial and well-defined internal structure, as explicitly seen in eq. (3.23). In particular, the MC PDFs, $D_{\mathrm{MC}}^{F, B}$, result from the Markovian process and thanks to kinematical mappings they respect the phase-space constraints exactly. Therefore, they are not equal to the standard DGLAP parton distributions functions, in particular they integrate emissions up the absolute phase-space limit, cf. eqs. (2.4) and (2.10), rather than stopping at some arbitrary scale $Q^{2}=\mu_{F}^{2}$.

The reader may check, analyzing one and two emissions in a detail, that the effect of the above "kinematical coupling" of the two showers through the variable $s_{i j}$ is conveniently absorbed by the construction of the four-momenta defined in section 2.2, hence eq. (3.22) is equivalent to eq. (3.19) up to the $\mathrm{N}^{2} \mathrm{LO}$ level, i.e. neglecting the $\mathrm{N}^{3} \mathrm{LO}$ and higher corrections. The above equivalence could also be tested numerically, similarly as was done in ref. [19].

Why do we insist on the FEV representation of PSMC knowing that any typical PSMC is built using the BEV algorithm? The important reason is that in any methodology of combining fixed-order perturbative corrections with PSMC one has to make an algebraic contact with the standard diagrammatic perturbative calculations, including factorization theorems, resummation techniques, etc., which are all defined within the standard Lorentzinvariant phase space (LIPS). The FEV parton shower works directly within the LIPS, ${ }^{16}$

\footnotetext{
${ }^{14}$ In the PSMC jargon this is referred to as a recoil mechanism.

${ }^{15}$ See ref. [36] for the precise formal derivation of the T.O. exponent from the Markovian FEV algorithm.

${ }^{16}$ Modulo kinematical mappings, effective beam technique, recoils, etc.
} 
while in the BEV algorithm, the relation between LIPS and the PSMC distributions is obscured due to the presence of ratios of the PDFs in the BEV distributions. The way out is to define a framework in which the BEV and FEV distributions are by construction exactly identical. This requires certain non-trivial extra care and effort. We shall follow this path in the following.

The PDF of eq. (3.23) looks pretty standard, for instance, it is directly implementable in form of the FEV Markovian MC. Its peculiarity is already quite obvious for a single emission $n=1$, where the upper phase-space boundary limit is not just regular $q_{1}^{2}<\hat{s}$, but more complicated $q_{1}^{2}<\left(1-z_{1}\right)^{2} \hat{s} / z_{1}$, reflecting the realistic phase-space boundary of the hard process, while the lower boundary is just regular $q_{1}^{2}>q_{s}^{2}$, i.e. the same as from the ordering in PSMC. This peculiarity influences mainly the first emissions, closest to the hard process, ${ }^{17}$ as discussed in section 2 . The consequence of the above peculiarity is that for our aim of the rigorous correspondence of the multiparton distributions between the FEV to BEV algorithms we need an auxiliary PDF with two competing factorization scales, $Q^{2}$ and $\hat{s}$ :

$$
\begin{aligned}
\bar{D}_{\mathrm{MC}}^{F}\left(\hat{s} \mid Q^{2}, x_{F}\right) & =\bar{D}^{F}\left(\hat{s} \mid q_{s}^{2}, x_{F}\right) e^{-S_{F}\left(\hat{s} \mid Q^{2}, q_{s}^{2}\right)}+\sum_{n=1}^{\infty} \int e^{-S_{F}\left(\hat{s} \mid Q^{2}, q_{1}^{2}\right)} \\
\times & \prod_{i=1}^{n}\left\{d^{3} \rho_{i}^{F}\left(s_{i}\right) e^{-S_{F}\left(s_{i} \mid q_{i}^{2}, q_{i+1}^{2}\right)} \theta_{Q^{2}>q_{i-1}^{2}>q_{i}^{2}>q_{s}^{2}}\right\} \bar{D}^{F}\left(\hat{s} \mid q_{s}^{2}, x_{s}\right) \delta_{x_{F}=x_{s} \prod_{j=1}^{n} z_{j}}
\end{aligned}
$$

where $q_{n+1} \equiv q_{s}$ and it coincides with the PDF of eq. (3.19) for $Q^{2}=s=\hat{s} / x_{F}$, i.e.

$$
D_{\mathrm{MC}}^{F}\left(\hat{s}, x_{F}\right)=\bar{D}_{\mathrm{MC}}^{F}\left(\hat{s} \mid \hat{s} / x_{F}, x_{F}\right)=\bar{D}_{\mathrm{MC}}^{F}\left(\hat{s} \mid \infty, x_{F}\right) .
$$

The main difference between this new PDF and the one introduced earlier in eq. (3.23) is the $\theta_{Q^{2}>q_{i-1}^{2}>q_{i}^{2}>q_{s}^{2}}$ function, see figure 3 , that restrict emissions only to the region below the scale $Q^{2}$, which in principle is an arbitrary parameter, as long as $Q^{2} \ll \hat{s}$. In that sense the new PDFs, $\bar{D}_{\mathrm{MC}}^{F, B}$, are closer to the standard DGLAP parton distribution functions.

With the above definitions we may work out the BEV algorithm providing the LO distributions, which by construction are exactly the same as from the FEV algorithm: ${ }^{18}$

$$
\begin{aligned}
d \sigma_{n_{F} n_{B}}^{\mathrm{LO}}= & \frac{d \sigma}{d \Omega}\left(s x_{F} x_{B}, \hat{\theta}\right) \prod_{i=1}^{n_{F}}\left\{d^{3} \omega_{i}^{F} \theta_{q_{i-1}^{2}>q_{i}^{2}}\right\} \prod_{j=1}^{n_{B}}\left\{d^{3} \omega_{j}^{B} \theta_{q_{j-1}^{2}>q_{j}^{2}}\right\} \\
& \times e^{-\Delta_{F}\left(x_{n_{F}}^{F} \mid q_{n_{F}}^{2}, q_{s}^{2}\right)} e^{-\Delta_{B}\left(x_{n_{B}}^{B} \mid q_{n_{B}}^{2}, q_{s}^{2}\right)} D^{F}\left(\hat{s}, x_{F}\right) D^{B}\left(\hat{s}, x_{B}\right) d x_{F} d x_{B} d \Omega, \\
x_{i}^{F}= & x_{F} / Z_{i}^{F}, x_{j}^{B}=x_{B} / Z_{j}^{B}, \hat{s}=s x_{F} x_{B},
\end{aligned}
$$

where $x_{i}^{F}=\prod_{m=1}^{i} z_{m}$ includes $z_{i}$ from $d^{3} \omega_{i}^{F}$ and similarly for $x_{j}^{B}$ from $d^{3} \omega_{i}^{B}$. The single-

\footnotetext{
${ }^{17}$ Already for the second emission, the limit $q_{2}^{2}<q_{1}^{2}$ is more important than $q_{2}^{2}<\left(1-z_{2}\right)^{2} \hat{s} /\left(z_{1} z_{2}\right)$, which can be neglected modulo NNLO.

${ }^{18}$ Modulo the kinematical coupling mentioned above.
} 
emission distributions and form factors are defined as follows:

$$
\begin{aligned}
d^{3} \omega_{i}^{H} & =\frac{d q_{i}^{2} d z_{i}}{q_{i}^{2}} \frac{d \phi_{i}}{2 \pi} \mathbb{K}_{\mathrm{MC}}\left(x_{i-1} \mid z_{i}, q_{i}^{2}\right) e^{-\Delta_{\mathrm{MC}}\left(x_{i-1} \mid q_{i}^{2}, q_{i-1}^{2}\right)}, \\
\mathbb{K}_{\mathrm{MC}}\left(x^{*} \mid z, q^{2}\right) & \equiv \mathcal{P}\left(z_{i}\right) \theta_{(1-z)^{2} s x^{*} / z>q^{2}} \frac{\bar{D}_{\mathrm{MC}}\left(s x^{*} / z \mid q^{2}, x^{*} / z\right)}{\bar{D}_{\mathrm{MC}}\left(s x^{*} \mid q^{2}, x^{*}\right)}, \\
\Delta_{\mathrm{MC}}\left(x^{*} \mid q_{j-1}^{2}, q_{j}^{2}\right) & \equiv \int_{q_{j}^{2}}^{q_{j-1}^{2}} \frac{d q^{2}}{q^{2}} \int_{x^{*}}^{1} \frac{d z}{z} \mathbb{K}_{\mathrm{MC}}\left(x^{*} \mid z, q^{2}\right),
\end{aligned}
$$

and the shower label ${ }_{H}={ }_{F},{ }_{B}$ was temporarily omitted wherever it was unambiguous to do so.

Although it may not look obvious, the integrated cross section

$$
\sigma_{\mathrm{MC}}^{\mathrm{LO}}=\sum_{n_{F}, n_{B}=0}^{\infty} \int d \sigma_{n_{F} n_{B}}^{\mathrm{LO}}
$$

and all the multiparton distributions are exactly the same as in eqs. (3.22) and (3.23). Let us present a sketchy proof of that. ${ }^{19}$ Directly from the BEV algorithm for the PDF of eq. (3.23) of the showering quark we get

$$
\begin{aligned}
& \left.D_{\mathrm{MC}}^{F}\left(\hat{s}, x_{F}\right)\right|_{B E V}=\bar{D}_{\mathrm{MC}}(s \hat{x} \mid s, \hat{x})\left\{e^{-\Delta_{\mathrm{MC}}\left(s, q_{s}^{2} \mid x_{F}\right)}+\right. \\
& \left.+\sum_{n=1}^{\infty}\left[\prod_{i=1}^{n} \int_{q_{s}^{2}}^{q_{i-1}^{2}} \frac{d q_{i}^{2}}{q_{i}^{2}} \int_{x_{i-1}}^{1} \frac{d z_{i}}{z_{i}} e^{-\Delta_{\mathrm{MC}}\left(x_{i-1} \mid q_{i}^{2}, q_{i-1}^{2}\right)} \mathbb{K}_{\mathrm{MC}}\left(x_{i-1} \mid z_{i}, q_{i}^{2}\right)\right] e^{-\Delta_{\mathrm{MC}}\left(x_{n} \mid s, q_{n}^{2}\right)}\right\} .
\end{aligned}
$$

In order to prove that the above PDF is the same as from the FEV algorithm one exploit the fact that in the T.O. exponential representation of the double-scale PDF of eq. (3.24) we may detach (factorize off) the first emission, obtaining the following integral equation:

$$
\begin{aligned}
& \bar{D}_{\mathrm{MC}}^{F}\left(\hat{s} \mid Q^{2}, x\right)=\bar{D}^{F}\left(\hat{s} \mid q_{s}^{2}, x\right) e^{-S_{F}\left(\hat{s} \mid Q^{2}, q_{s}^{2}\right)}+ \\
& +\int_{q_{s}^{2}}^{Q^{2}} \frac{d q_{1}^{2}}{q_{1}^{2}} \int_{0}^{1} d x_{1} \int_{0}^{1} d z_{1} \mathcal{P}\left(z_{1}\right) \theta_{\left(1-z_{1}\right)^{2} \hat{s} / z_{1}>q_{1}^{2}} e^{-S_{F}\left(\hat{s} \mid Q^{2}, q_{1}^{2}\right)} \bar{D}_{\mathrm{MC}}\left(\hat{s} / z_{1} \mid q_{1}^{2}, x_{1}\right) \delta_{x=z_{1} x_{1}}
\end{aligned}
$$

Differentiating both sides by $\partial / \partial\left(\ln q_{s}^{2}\right)$ and then integrating $\int_{\ln q_{a}^{2}}^{\ln q_{b}^{2}} d\left(\ln q_{s}^{2}\right)$, one obtains

$$
e^{-S_{\mathrm{MC}}\left(\hat{s} \mid q_{b}^{2}, q_{a}^{2}\right)}=e^{-\Delta_{\mathrm{MC}}\left(x \mid q_{b}^{2}, q_{a}^{2}\right)} \frac{\bar{D}_{\mathrm{MC}}\left(\hat{s} \mid q_{b}^{2}, x\right)}{\bar{D}_{\mathrm{MC}}\left(\hat{s} \mid q_{a}^{2}, x\right)} .
$$

The above identity can be used many times in order to eliminate the ratios of the PDFs in the BEV formula and transform without any approximation the BEV distributions of eq. (3.28) into the FEV distributions of eq. (3.23).

\footnotetext{
${ }^{19}$ The result of the detailed proof is the same, of course.
} 


\subsection{NLO weight in parton shower and its algebraic validation}

Having defined the framework in which the FEV and BEV distributions of LO PSMC are identical, we may define a MC weight correcting the hard process in the (notoriously) inefficient FEV LO PSMC, but having advantage of a straightforward connection to the perturbative expansion, and apply it within LO PSMC implemented using the efficient BEV algorithm. The NLO completeness of such a scheme can be proven analytically within FEV without any troubles, due to the absence of the 'obscure' ratios of the PDFs of BEV. The only extra cost is that in order to gain a perfect FEV $\leftrightarrow$ BEV compatibility, we had to introduce the special LO PDFs $\bar{D}\left(\hat{s} \mid Q^{2}, x\right)$ with two factorization scales, at least for the purpose of the discussion of the NLO completeness of the KrkNLO scheme, while in practice we shall be able to obtain $\bar{D}$ from the standard $\overline{\mathrm{MS}}$ PDFs

The differential cross section in which the hard process is NLO-corrected for the $q \bar{q}$ channel in the KrkNLO method reads as follows: ${ }^{20}$

$$
d \sigma_{n_{F} n_{B}}^{\mathrm{NLO}}=\left(1+\Delta_{V S}+\sum_{i=1}^{n_{F}} W_{q \bar{q}}^{[1]}\left(\tilde{\alpha}_{i}^{F}, \tilde{\beta}_{i}^{F}\right)+\sum_{j=1}^{n_{B}} W_{q \bar{q}}^{[1]}\left(\tilde{\alpha}_{j}^{B}, \tilde{\beta}_{j}^{B}\right)\right) d \sigma_{n_{F} n_{B}}^{\mathrm{LO}},
$$

where

$$
W_{q \bar{q}}^{[1]}=\frac{d^{5} \bar{\beta}_{q \bar{q}}}{d^{5} \sigma_{q \bar{q}}^{\mathrm{L} \bar{O}}}=\frac{d^{5} \sigma_{q \bar{q}}^{\mathrm{NLO}}-d^{5} \sigma_{q \bar{q}}^{\mathrm{LO}}}{d^{5} \sigma_{q \bar{q}}^{\mathrm{LO}}}=W_{q \bar{q}}^{(1)}-1,
$$

see eqs. (3.6) and (3.10). The structure of this weight, with the summation over the emitted gluons, is a straightforward generalization of the weight used in many MC programs with multiphoton exponentiated corrections, see for instance ref. [37].

As pointed out in ref. [20], in order to get the complete NLO corrections to the hard process, it is enough to retain in the sum in eq. (3.31) only a single term, the one with the maximum $k_{T}^{2}$. Since our $q^{2}$-variable is practically identical to $k_{T}^{2}$ we may retain only one term for a gluon with the maximum $q_{i_{F}}^{2}$ or $q_{j_{B}}^{2}$, from one of the two showers. In the case of the BEV algorithm with competition, this gluon is just the one which was generated first.

Similarly, as we have detached the first emission from the T.O. exponential representation of the PDF in eq. (3.29), we now factorize off explicitly the gluon emissions with the maximum $q_{F}^{2}$ and $q_{B}^{2}$ from the product of two PDFs in eq. (3.22):

$$
\begin{aligned}
& D_{\mathrm{MC}}^{F}\left(\hat{s}, \hat{x}_{F}\right) D_{\mathrm{MC}}^{B}\left(\hat{s}, \hat{x}_{B}\right)=e^{-S_{F}\left(\hat{s} \mid s_{1_{F}}, q_{s}^{2}\right)} e^{-S_{B}\left(\hat{s} \mid s_{1_{B}}, q_{s}^{2}\right)} \bar{D}_{\mathrm{MC}}^{F}\left(\hat{s} \mid q_{s}^{2}, \hat{x}_{F}\right) \bar{D}_{\mathrm{MC}}^{B}\left(\hat{s} \mid q_{s}^{2}, \hat{x}_{B}\right) \\
& +\int d^{3} \rho_{1}^{F}\left(\hat{s} / z_{1_{F}}\right) e^{-S_{F}\left(\hat{s} \mid s_{1_{F}}, q_{1_{F}}^{2}\right)} e^{-S_{B}\left(\hat{s} \mid s_{1_{B}}, q_{1_{F}}^{2}\right)} \frac{1}{z_{1_{F}}} \bar{D}_{\mathrm{MC}}^{F}\left(\hat{s} \mid q_{1_{F}}^{2}, \hat{x}_{F} / z_{1_{F}}\right) \bar{D}_{\mathrm{MC}}^{B}\left(\hat{s} \mid q_{1_{F}}^{2}, \hat{x}_{B}\right) \\
& q_{s}^{2}<q_{1_{F}}^{2}<s_{1_{F}} \\
& +\int d^{3} \rho_{1}^{B}\left(\hat{s} / z_{1_{B}}\right) e^{-S_{F}\left(\hat{s} \mid s_{1_{F}}, q_{1_{B}}^{2}\right)} e^{-S_{B}\left(\hat{s} \mid s_{1_{B}}, q_{1_{B}}^{2}\right)} \bar{D}_{\mathrm{MC}}^{F}\left(\hat{s} \mid q_{1_{B}}^{2}, \hat{x}_{F}\right) \frac{1}{z_{1_{B}}} \bar{D}_{\mathrm{MC}}^{B}\left(\hat{s} \mid q_{1_{B}}^{2}, \hat{x}_{B} / z_{1_{B}}\right), \\
& q_{s}^{2}<q_{1_{B}}^{2}<s_{1_{B}}
\end{aligned}
$$

where $s_{1_{F}}=\hat{s} / \hat{x}_{F}$ and $s_{1_{B}}=\hat{s} / \hat{x}_{B}$.

\footnotetext{
${ }^{20}$ We adopt a convention that $\sum_{n=1}^{2} d_{n}=0$.
} 
In order to improve readability, we are going to omit temporarily (until eq. (3.38)) the argument $\hat{s}$ in the $\bar{D}_{\mathrm{MC}}^{F, B}(\hat{s} \mid \ldots)$ and the $S_{F, B}(\hat{s} \mid \ldots)$ functions. Moreover, the "kinematical coupling" of the two showers is will be restored, that is we are going to replace in the following $s_{1_{F}}, s_{1_{F}} \rightarrow s=\hat{s} /\left(x_{F} x_{B}\right)$. The above decomposition is easily exploited in calculating the NLO cross section with the MC weight of eq. (3.31) truncated to a single term $W_{q \bar{q}}^{[1]}$ with the maximum $q^{2}$ :

$$
\begin{aligned}
& \sigma^{\mathrm{NLO}}[J]=\sum_{n_{F}, n_{B}=1}^{\infty} \int d \sigma_{n_{F} n_{B}}^{\mathrm{NLO}}=\int d x_{F} d x_{B} d \Omega \frac{d \sigma}{d \Omega}\left(s x_{F} x_{B}, \hat{\theta}\right)\left(1+\Delta_{V S}\right) e^{-S_{F}\left(s, q_{s}^{2}\right)} e^{-S_{B}\left(s, q_{s}^{2}\right)} \\
& \times \bar{D}_{\mathrm{MC}}^{F}\left(q_{s}^{2}, x_{F}\right) \bar{D}_{\mathrm{MC}}^{B}\left(q_{s}^{2}, x_{B}\right) J_{\mathrm{LO}}\left(x_{F}, x_{B}\right) \\
& +\int d x_{F} d x_{B} d \Omega\left\{\int_{q_{s}^{2}<q_{1_{F}^{2}}^{2}<s} d^{3} \rho_{1}^{F}\left(s x_{F} x_{B}\right)\left(1+\Delta_{V S}+W_{q \bar{q}}^{[1]}\left(k_{1}\right)\right) \frac{d \sigma}{d \Omega}\left(s x_{F} x_{B} z_{1_{F}}, \hat{\theta}\right)\right. \\
& \times e^{-S_{F}\left(s, q_{1_{F}}^{2}\right)} e^{-S_{B}\left(s, q_{1_{F}}^{2}\right)} \bar{D}_{\mathrm{MC}}^{F}\left(q_{1_{F}}^{2}, x_{F}\right) \bar{D}_{\mathrm{MC}}^{B}\left(q_{1_{F}}^{2}, x_{B}\right) J_{\mathrm{NLO}}\left(x_{F}, x_{B}, z_{1_{F}}, k_{1 T}^{2}\right) \\
& +\int d^{3} \rho_{1}^{B}\left(s x_{F} x_{B}\right)\left(1+\Delta_{V S}+W_{q \bar{q}}^{[1]}\left(k_{1}\right)\right) \frac{d \sigma}{d \Omega}\left(s x_{F} x_{B} z_{1_{B}}, \hat{\theta}\right) \\
& q_{s}^{2}<q_{1_{B}}^{2}<s \\
& \left.\times e^{-S_{F}\left(s, q_{1_{B}}^{2}\right)} e^{-S_{B}\left(s, q_{1_{B}}^{2}\right)} \bar{D}_{\mathrm{MC}}^{F}\left(q_{1_{B}}^{2}, x_{F}\right) \bar{D}_{\mathrm{MC}}^{B}\left(q_{1_{B}}^{2}, x_{B}\right) J_{\mathrm{NLO}}\left(x_{F}, x_{B}, z_{1_{B}}, k_{1 T}^{2}\right)\right\},
\end{aligned}
$$

where we have introduced $x_{F}$ and $x_{B}$ before the emission, as in a typical fixed-order NLO calculation. They are related to $\hat{x}_{F}$ and $\hat{x}_{B}$ from eq. (3.33) in such a way that if the emission happens on the leg $F$, then $x_{F}=\hat{x}_{F} / z_{1_{F}}$ and $x_{B}=\hat{x}_{B}$. And symmetrically if emission occurs on the leg $B$. The same expression we can get directly from distributions of the BEV algorithm, as shown explicitly in appendix A.

For the purpose of the proof of compatibility of the above formula with the fixedorder calculation (MCFM) for the entire class of the LO and NLO observables, we have introduced in the above the jet functions, $J_{\mathrm{LO}}\left(x_{F}, x_{B}\right)$ and $J_{\mathrm{NLO}}\left(x_{F}, x_{B}, z_{1}, k_{1 T}^{2}\right)$, which satisfy the following properties:

$$
J_{\mathrm{NLO}}\left(x_{F}, x_{B}, z_{1}, k_{1 T}^{2}\right) \rightarrow J_{\mathrm{LO}}\left(x_{F}, x_{B}\right) \quad \text { for } \quad k_{1 T}^{2} \rightarrow 0, \quad \text { or } \quad z_{1} \rightarrow 1,
$$

often referred to as an infra-red safety requirement. In the experimental practice, the function $J_{\mathrm{NLO}}$ represents the most general 2-dimensional histogramming in the transverse momentum of the gluon (or the heavy boson) and the $z_{1}$ variable, related to the rapidity difference between the heavy boson and the emitted gluon (or the ratio of the effective mass of the heavy boson and the heavy boson plus gluon system). The meaning of the above limit $k_{1 T}^{2} \rightarrow 0$ is that for the first bin in $k_{1 T}^{2}$, the one including the $k_{1 T}^{2}=0$ point, we are not allowed to do any binning in $z_{1}$ (we have to sum up inclusively over all $z_{1}$, similarly as for $J_{\mathrm{LO}}$ we sum up inclusively over all $k_{1 T}^{2}$ ).

In order to recover the fixed-order NLO formula of MCFM, all terms $\mathcal{O}\left(\alpha_{s}^{2}\right)$ have to be carefully eliminated. It is easy to do it for the $\sim d^{3} \rho W_{q \bar{q}}^{[1]}$ parts, which are formally 
$\mathcal{O}\left(\alpha_{s}^{1}\right)$. We replace in them $\bar{D}_{\mathrm{MC}}^{F}\left(\hat{s} \mid q_{1_{F}, 1_{B}}^{2}, x_{F}\right) \rightarrow \bar{D}_{\mathrm{MC}}^{F}\left(\hat{s} \mid s, x_{F}\right)$ and also eliminate safely the Sudakov exponents: ${ }^{21}$

$$
\begin{aligned}
\sigma^{\mathrm{NLO}}[J]= & \int d x_{F} d x_{B} d \Omega\left\{e^{-S_{F}\left(s, q_{s}^{2}\right)} e^{-S_{B}\left(s, q_{s}^{2}\right)} \bar{D}_{\mathrm{MC}}^{F}\left(q_{s}^{2}, x_{F}\right) \bar{D}_{\mathrm{MC}}^{B}\left(q_{s}^{2}, x_{B}\right)\right\} \\
& \times\left(1+\Delta_{V S}\right) \frac{d \sigma}{d \Omega}\left(s_{1}, \hat{\theta}\right) J_{\mathrm{LO}}\left(x_{F}, x_{B}\right) \\
& +\int d x_{F} d x_{B} d \Omega\left\{\int_{q_{s}^{2}<q_{1_{F}}^{2}<s} d^{3} \rho_{1}^{F}\left(s_{1}\right) e^{-S_{F}\left(s, q_{1_{F}}^{2}\right)} e^{-S_{B}\left(s, q_{1_{F}}^{2}\right) \bar{D}_{\mathrm{MC}}^{F}\left(q_{1_{F}}^{2}, x_{F}\right) \bar{D}_{\mathrm{MC}}^{B}\left(q_{1_{F}}^{2}, x_{B}\right)}\right. \\
& +\int d^{3} \rho_{1}^{B}\left(s_{1}\right) e^{-S_{F}\left(s, q_{1_{B}}^{2}\right)} e^{\left.-S_{B}\left(s, q_{1_{B}}^{2}\right) \bar{D}_{\mathrm{MC}}^{F}\left(q_{1_{B}}^{2}, x_{F}\right) \bar{D}_{\mathrm{MC}}^{B}\left(q_{1_{B}}^{2}, x_{B}\right)\right\}} \\
& q_{s}^{2}<q_{1_{B}}^{2}<s \\
& \times\left(1+\Delta_{V S}\right) \frac{d \sigma}{d \Omega}\left(s x_{F} x_{B} z_{1}, \hat{\theta}\right) J_{\mathrm{NLO}}\left(x_{F}, x_{B}, z_{1}, k_{1 T}^{2}\right) \\
& +\int d x_{F} d x_{B} d \Omega \int\left(d^{3} \rho_{1}^{F}\left(s_{1}\right)+d^{3} \rho_{1}^{B}\left(s_{1}\right)\right) W_{q \bar{q}}^{[1]}\left(k_{1}\right) \frac{d \sigma}{d \Omega}\left(s x_{F} x_{B} z_{1}, \hat{\theta}\right) \\
& \times \bar{D}_{\mathrm{MC}}^{F}\left(s, x_{F}\right) \bar{D}_{\mathrm{MC}}^{B}\left(s, x_{B}^{2}\right) J_{\mathrm{NLO}}\left(x_{F}, x_{B}, z_{1}, k_{1 T}^{2}\right),
\end{aligned}
$$

where we were also allowed to replace $z_{1_{F}}, z_{1_{B}} \rightarrow z_{1}$, because in terms of the Sudakov variables before the emission it is the same variable. We have also introduced $s_{1}=s x_{F} x_{B}$.

The remaining parts $\sim\left(1+\Delta_{V S}\right)$ are less trivial. In order to use again the identity of eq. (3.33) for folding in three integrals within the $\{\ldots\}$ braces back into a simple product of two PDFs, we have to do something with the $z_{1}$-dependence in $d \sigma / d \Omega$ and the $k_{1 T^{-}}^{2}$ dependence of the jet function $J_{\mathrm{NLO}}$ in the second part. The solution is to add and subtract a term proportional $\frac{d \sigma}{d \Omega}\left(s_{1}, \hat{\theta}\right) J_{\mathrm{LO}}\left(x_{F}, x_{B}\right)$ in the second part, regroup and use again eq. (3.33):

$$
\begin{aligned}
\sigma^{\mathrm{NLO}}[J]= & \int d x_{F} d x_{B} d \Omega \bar{D}_{\mathrm{MC}}^{F}\left(s, x_{F}\right) \bar{D}_{\mathrm{MC}}^{B}\left(s, x_{B}\right)\left(1+\Delta_{V S}\right) \frac{d \sigma}{d \Omega}\left(s_{1}, \hat{\theta}\right) J_{\mathrm{LO}}\left(x_{F}, x_{B}\right) \\
& +\int d x_{F} d x_{B} d \Omega\left\{\int d^{3} \rho_{1}^{F}\left(s_{1}\right) \bar{D}_{\mathrm{MC}}^{F}\left(q_{1_{F}}^{2}, x_{F}\right) \bar{D}_{\mathrm{MC}}^{B}\left(q_{1_{F}}^{2}, x_{B}\right)\right. \\
& \left.+\int d^{3} \rho_{1}^{B}\left(s_{1}\right) \bar{D}_{\mathrm{MC}}^{F}\left(q_{1_{B}}^{2}, x_{F}\right) \bar{D}_{\mathrm{MC}}^{B}\left(q_{1_{B}}^{2}, x_{B}\right)\right\} \\
& \times\left[\frac{d \sigma}{d \Omega}\left(s x_{F} x_{B} z_{1}, \hat{\theta}\right) J_{\mathrm{NLO}}\left(x_{F}, x_{B}, z_{1}, k_{1 T}^{2}\right)-\frac{d \sigma}{d \Omega}\left(s_{1}, \hat{\theta}\right) J_{\mathrm{LO}}\left(x_{F}, x_{B}\right)\right] \\
& +\int d x_{F} d x_{B} d \Omega \int d^{5} \rho_{q \bar{q}}^{L O} W_{q \bar{q}}^{[1]}\left(k_{1}\right) \bar{D}_{\mathrm{MC}}^{F}\left(s, x_{F}\right) \bar{D}_{\mathrm{MC}}^{B}\left(s, x_{B}\right) J_{\mathrm{NLO}}\left(x_{F}, x_{B}, z_{1}, k_{1 T}^{2}\right),
\end{aligned}
$$

where we have profited from finiteness of the integrals, in order to omit $\left(1+\Delta_{V S}\right)$ and the Sudakov exponents wherever possible and to set the lower integration limits of $q^{2}$ to zero.

\footnotetext{
${ }^{21}$ The collinear singularity in $q^{2} \rightarrow 0$ is killed by $W_{q \bar{q}}^{[1]}$, so $\int d q^{2} / q^{2} e^{-S\left(q_{2} \ldots\right)}$ cannot give a $\sim 1 / \alpha_{s}$ contribution.
} 
The final clean-up of $\mathcal{O}\left(\alpha_{s}^{2}\right)$ terms involves the change $q_{1_{F}}^{2} \rightarrow s$ and $q_{1_{B}}^{2} \rightarrow s$ in the PDFs in the second integral and recombining it with the third one:

$$
\begin{aligned}
\sigma^{\mathrm{NLO}}[J]= & \int d x_{F} d x_{B} d \Omega\left(1+\Delta_{V S}\right) \frac{d \sigma}{d \Omega}\left(s_{1}, \hat{\theta}\right) J_{\mathrm{LO}}\left(x_{F}, x_{B}\right) \bar{D}_{\mathrm{MC}}^{F}\left(\hat{s} \mid s, x_{F}\right) \bar{D}_{\mathrm{MC}}^{B}\left(\hat{s} \mid s, x_{B}\right) \\
& +\int d x_{F} d x_{B} d \Omega \int\left\{d^{5} \rho_{q \bar{q}}^{\mathrm{LO}}\left[1+W_{q \bar{q}}^{[1]}\left(k_{1}\right)\right] J_{\mathrm{NLO}}\left(x_{F}, x_{B}, z_{1}, k_{1 T}^{2}\right)\right. \\
& \left.-\left(d^{3} \rho_{1}^{F}+d^{3} \rho_{1}^{B}\right) \frac{d \sigma}{d \Omega}\left(s_{1}, \hat{\theta}\right) J_{\mathrm{LO}}\left(x_{F}, x_{B}\right)\right\} \bar{D}_{\mathrm{MC}}^{F}\left(\hat{s} \mid s, x_{F}\right) \bar{D}_{\mathrm{MC}}^{B}\left(\hat{s} \mid s, x_{B}\right)
\end{aligned}
$$

We may finally eliminate the MC weight of eq. (3.32) going back to the NLO distributions:

$$
\begin{aligned}
\sigma^{\mathrm{NLO}}[J]= & \int d x_{F} d x_{B} d \Omega\left(1+\Delta_{V S}\right) \frac{d \sigma}{d \Omega}\left(s_{1}, \hat{\theta}\right) J_{\mathrm{LO}}\left(x_{F}, x_{B}\right) D_{\mathrm{MC}}^{F}\left(\hat{s}, x_{F}\right) D_{\mathrm{MC}}^{B}\left(\hat{s}, x_{B}\right) \\
& +\int d x_{F} d x_{B} d \Omega\left\{d^{5} \rho_{q \bar{q}}^{\mathrm{NLO}} J_{\mathrm{NLO}}\left(x_{F}, x_{B}, z_{1}, k_{1 T}^{2}\right)-d^{5} \rho_{q \bar{q}}^{\mathrm{LO}} J_{\mathrm{LO}}\left(x_{F}, x_{B}\right)\right\} \\
& \times D_{\mathrm{MC}}^{F}\left(\hat{s}, x_{F}\right) D_{\mathrm{MC}}^{B}\left(\hat{s}, x_{B}\right),
\end{aligned}
$$

where $D_{\mathrm{MC}}^{F, B}(\hat{s}, x)$ of eq. (3.25) was also recovered.

The above looks like an example of the fixed-order NLO calculation formula employing the technique of soft-collinear counter-terms following the Catani-Seymour (CS) work [24], with the explicit definition of an arbitrary NLO observable using the $J$-function. For the sake of completeness, such a formula in the standard $\overline{\mathrm{MS}}$ scheme using the CS method is shown explicitly in eq. (B.7) in appendix B for the same $q \bar{q}$ channel.

However, there are two important differences between the formulas in eq. (B.7) and in the above eq. (3.39): missing the $\sim \delta\left(k_{1 T}^{2}\right) \Sigma$ term and non- $\overline{\mathrm{MS}}$ PDFs in eq. (3.39). The real emission integral with subtraction, in spite of slightly different notation, is identical. These differences are, of course, due to the differences between the MC and $\overline{\mathrm{MS}}$ factorization schemes, and are well understood, see the discussion in the following section 4 and in appendix B. The important bonus of the comparison of eqs. (B.7) and (3.39) is that we can establish the relation between the quark PDF used in the parton shower MC and the corresponding PDF of the $\overline{\mathrm{MS}}$ scheme, modulo NNLO, in a solid and unambiguous way:

$$
f_{q}^{\mathrm{MC}}\left(Q^{2}, x\right)=D_{\mathrm{MC}}\left(Q^{2}, x\right)=\bar{D}_{\mathrm{MC}}\left(Q^{2} \mid Q^{2} / x, x\right)
$$

where $f_{q}^{\mathrm{MC}}$ is the quark $\mathrm{PDF}$ in the MC scheme defined in eq. (3.17).

In this way we have proven algebraically that the KrkNLO scheme is equivalent to the fixed-order $N L O$ calculation in the entire functional space of the NLO-class experimental observables. ${ }^{22}$

\subsection{Summarizing the KrkNLO method}

In the following, we summarize and comment on the key elements of the KrkNLO method, starting from the $q \bar{q}$ channel only and then adding the $q g$ channel.

\footnotetext{
${ }^{22}$ It seems that this kind of an explicit rigorous, albeit tedious, algebraic proof is not available for the other methods of combining the NLO corrections with the LO parton shower.
} 
1. The first element is the reorganization of the NLO corrections, the same as ref. [19]. Starting from the standard $\overline{\mathrm{MS}}$ calculation, a strictly collinear part of the NLO corrections is removed thanks to the clever redefinition of the LO PDFs from the $\overline{\mathrm{MS}}$ scheme to the MC scheme, see eq. (3.17). In a nutshell, in the standard $\overline{\mathrm{MS}}$ procedure, one subtracts from the diagrammatic real + virtual results a pure $\sim P(z) / \epsilon$ pole, while in the case of KrkNLO one subtracts the $\Gamma_{\mathrm{MC}}(z, \epsilon)$ function, which contains extra non-pole terms. As discussed in ref. [19], see also eq. (B.11), this $\Gamma_{\mathrm{MC}}$ function is just the integral over the LO distribution of the single-gluon emission in the parton shower MC, ${ }^{23}$ written in $4+\epsilon$ dimension, together with the Sudakov form factor, obeying typical parton shower sum rules. This element of the KrkNLO prescription remains the same as in ref. [19], provided the LO distribution is the same as in the present study. This assumption is valid because the LO distribution of eq. (3.3) is indeed the same in ref. [19] and in the Catani-Seymour inspired implementations of the single-gluon emissions in Sherpa and Herwig ++ . What remains to be checked is whether the upper phase-space limit, $\alpha+\beta \leq 1$, is not spoiled in a given PSMC by the use of the backward evolution algorithm. We are going to come back to this point shortly.

2. The second element of the KrkNLO prescription is the construction, implementation, and algebraic validation of the multiplicative weight introducing the NLO corrections in the multiparton environment of PSMC. The weight of eq. (3.6) is implemented following eq. (3.31). The summation over gluons is necessary only for the angular ordering, while for the $q^{2}$-ordering or $k_{T}$-ordering it is enough to keep just one term for the gluon next to the hard process. Validity of above method can be proven rigorously, see section 3.4, in the case when the initial-state PSMC is realized using the forward Markovian evolution (FEV) algorithm, in the presence of any observabledefining function $J$, see eq. (3.35). Such a FEV algorithm would be terribly inefficient for the resonant $Z$-boson production, but it is mathematically perfectly well defined. (In such a gedanken FEV scenario with the complete phase-space coverage, the output $\mathrm{PDF}$ from the MC at the hard process scale will be automatically in the MC scheme, following eq. (3.17), provided that the input PDF at low $q_{s}^{2}$ is in the $\overline{\mathrm{MS}}$ scheme. ${ }^{24}$ )

3. The above validation proof of the NLO weight has to be extended to the (efficient) backward evolution (BEV) scenario of the typical PSMC, such as Sherpa or Herwig ++ . It was shown in section 3.3 that this is feasible, by means of formulating the twin FEV and BEV algorithms, using the same PDFs, which produce exactly the same multiparton exclusive distributions. What is highly non-trivial is that the full phase-space coverage is not lost. The price for this was that we had to introduce the auxiliary $\operatorname{PDF} \bar{D}\left(\hat{s} \mid Q^{2}, x\right)$ of eq. (3.24) with two competing factorization scales, in addition to the single-scale PDF of eq. (3.23). Luckily, at the end this

\footnotetext{
${ }^{23}$ It is the so-called soft-collinear counter-term. Although it is defined with the help of PSMC for the DY process, its definition is universal.

${ }^{24}$ In other words, such a FEV MC performs not only the LO DGLAP evolution but also the transition from one to another factorization scheme.
} 
auxiliary PDF can be finally eliminated from the BEV algorithm. Its main role is to provide theoretical control for the $\mathrm{FEV} \rightarrow \mathrm{BEV}$ transition, desired in view of the previous point.

4. The elimination of the auxiliary parton distribution function $\bar{D}\left(\hat{s} \mid Q^{2}, x\right)$ can be seen best with the analysis presented in appendix $\mathrm{A}$, where the transition $\mathrm{FEV} \rightarrow \mathrm{BEV}$ is analyzed in a fine detail for $n=0,1$ emissions. In eq. (A.1) for $n=0$, we see that we may replace $\bar{D}(\hat{s} \mid s, x) \rightarrow D(\hat{s}, x)$ using eq. (3.25). For $n=1$ in eq. (A.6), one may show that, modulo $\mathcal{O}\left(\alpha_{s}^{2}\right)$ terms, the ratio of $\bar{D}$-functions can be replaced with any kind of the LO PDFs and the exponents, $\exp (-\Delta)$, can be neglected. What cannot be approximated there, it is the exact implementation of the evolution kernel, keeping the correct phase-space limits due to the $\theta$-functions. This is, however, not the problem, because any standard BEV implementation with the veto algorithm does that correctly. ${ }^{25}$ We conclude that, in the BEV scenario, all $\bar{D}$-functions go away and only the single-scale PDFs in the MC factorization scheme seen in eq. (A.1) are left finally in the game. ${ }^{26}$

In the above analysis we have omitted $q g$ channel. For the $Z / \gamma^{*}$-boson production process however, is not difficult to include it following the same steps described above. ${ }^{27}$ In our implementation of the KrkNLO method in PSMC, whenever in the first step of the BEV PS algorithm the transition from the quark (antiquark) to the gluon is generated, we associate with such an event the weight computed according to eqs. (3.8) and (3.9). This weight corresponds to observables averaged over the $Z / \gamma^{*}$-boson decay angles and is sufficient for the current paper, as we are going to concentrate on the $Z / \gamma^{*}$-boson transverse momentum and rapidity distributions. The discussion of the $Z$-decay leptonic observables, as well as more details on the implementation of the $q g$ channel, is reserved for a separate publication.

\section{Fixed-order NLO benchmarks}

For the numerical evaluation of the cross sections ${ }^{28}$ at the LHC for the proton-proton collision energy of $\sqrt{s}=8 \mathrm{TeV}$ we have chosen the following set of Standard Model (SM) input parameters:

$$
\begin{array}{rlrl}
M_{Z} & =91.1876 \mathrm{GeV}, & \Gamma_{Z} & =2.4952 \mathrm{GeV}, \\
M_{W} & =80.4030 \mathrm{GeV}, & \Gamma_{W} & =2.1240 \mathrm{GeV}, \\
G_{\mu} & =1.16637 \times 10^{-5} \mathrm{GeV}^{-2}, & m_{t} & =173.2 \mathrm{GeV}, \\
\alpha_{s}\left(M_{Z}^{2}\right) & =0.13938690, &
\end{array}
$$

\footnotetext{
${ }^{25}$ More detailed analysis of this kind was done but is left beyond the scope of the present paper.

${ }^{26}$ One can show algebraically that the effective PDF generated by the above BEV at low $q_{s}^{2}$ will be in the $\overline{\mathrm{MS}}$ scheme - the BEV algorithm is not only undoing the LO evolution, but also the transition from the $\overline{\mathrm{MS}}$ to $\mathrm{MC}$ scheme.

${ }^{27}$ All ingredient distributions are well known in the literature.

${ }^{28}$ Unless stated otherwise in the text.
} 


\begin{tabular}{|c||c|c|c|}
\hline & MCFM & Sherpa & Herwig++ \\
\hline$\sigma_{\text {tot }}[\mathrm{pb}]$ & $936.9 \pm 0.1$ & $937.2 \pm 0.2$ & $937.0 \pm 0.6$ \\
\hline
\end{tabular}

Table 1. Values of the total cross section with statistical errors at the Born level for the Drell-Yan process in the $\overline{\mathrm{MS}}$ scheme.

and the $G_{\mu}$-scheme [38] for the electroweak sector of the Standard Model. To compute the hadronic cross section we also use the MSTW2008 LO set of parton distribution functions [39], and take the renormalization and factorization scales to be $\mu_{R}^{2}=\mu_{F}^{2}=M_{Z}^{2}$. The only detector acceptance cuts are imposed on the invariant mass of the final-state lepton pair $\left(Z / \gamma^{\star}\right.$-boson):

$$
50 \mathrm{GeV}<M_{l \bar{l}}<150 \mathrm{GeV} .
$$

In order to check that our settings are identical in all used programs, we began with the comparisons at the Born level. The results presented in table. 1 show a very good agreement (within statistical errors) between different programs.

\subsection{PDFs in MC scheme}

In section 3.2, we have introduced the MC factorization scheme and explained why this scheme is better suited for matching the NLO results with the parton shower than the standard $\overline{\mathrm{MS}}$ scheme.

The MC factorization scheme comes with a new set of parton distribution functions that can be obtained from the standard $\overline{\mathrm{MS}}$ PDFs via the relations given in eqs. (3.17) and (3.18). Note that the convolution terms in eq. (3.17) introduce the dependence on the renormalization scale via the strong coupling multiplying the expressions for $\Delta C_{2 q}$ and $\Delta C_{2 g}$ in eqs. (3.13) and (3.16). In what follows, we shall always choose that scale to be equal to the scale $Q^{2}=\mu_{F}^{2}$ of the $\overline{\mathrm{MS}} \mathrm{PDF}$.

For clarity of the discussion, before presenting the complete NLO corrections to the Drell-Yan process, we shall be starting in what follows from results limited to the pure $q \bar{q}$ channel. This case corresponds to setting the gluon coefficient functions of eqs. (3.14) and (3.15) to zero. That in turn implies that, for the two schemes to be consistent at $\mathcal{O}\left(\alpha_{s}\right)$, the last term in the relation between the quark PDFs in the $\overline{\mathrm{MS}}$ and MC schemes from eq. (3.17) should be omitted.

The MC PDFs for light quarks and light antiquarks, obtained from the MSTW2008 LO set [39] are shown in figure 7 through ratios to the $\overline{\mathrm{MS}}$ PDFs. The curves correspond to the choice of $Q^{2}=100 \mathrm{GeV}$. The same scale was used as the argument of $\alpha_{s}$. Each panel shown in figure 7 contains two curves. The solid line anticipates including both the $q \bar{q}$ and $q g$ channels, hence it was obtained exactly with the formula (3.17). On the other hand, the dotted line corresponds to the case of the pure $q \bar{q}$ channel, hence only the convolution with $\Delta C_{2 q}$ was used, as explained earlier.

As seen in figure 7, the MC PDFs without the gluon, e.g. without the last term in eq. (3.17), are very similar to the $\overline{\mathrm{MS}}$ quarks at low and moderate $x$. The MC PDFs in this region are only about $2 \%$ higher. At large $x$ the ratio increases, reaching the value $2-3$ near $x=1$. Note that the MC PDFs are always higher than the standard $\overline{\mathrm{MS}}$ quark PDFs, 

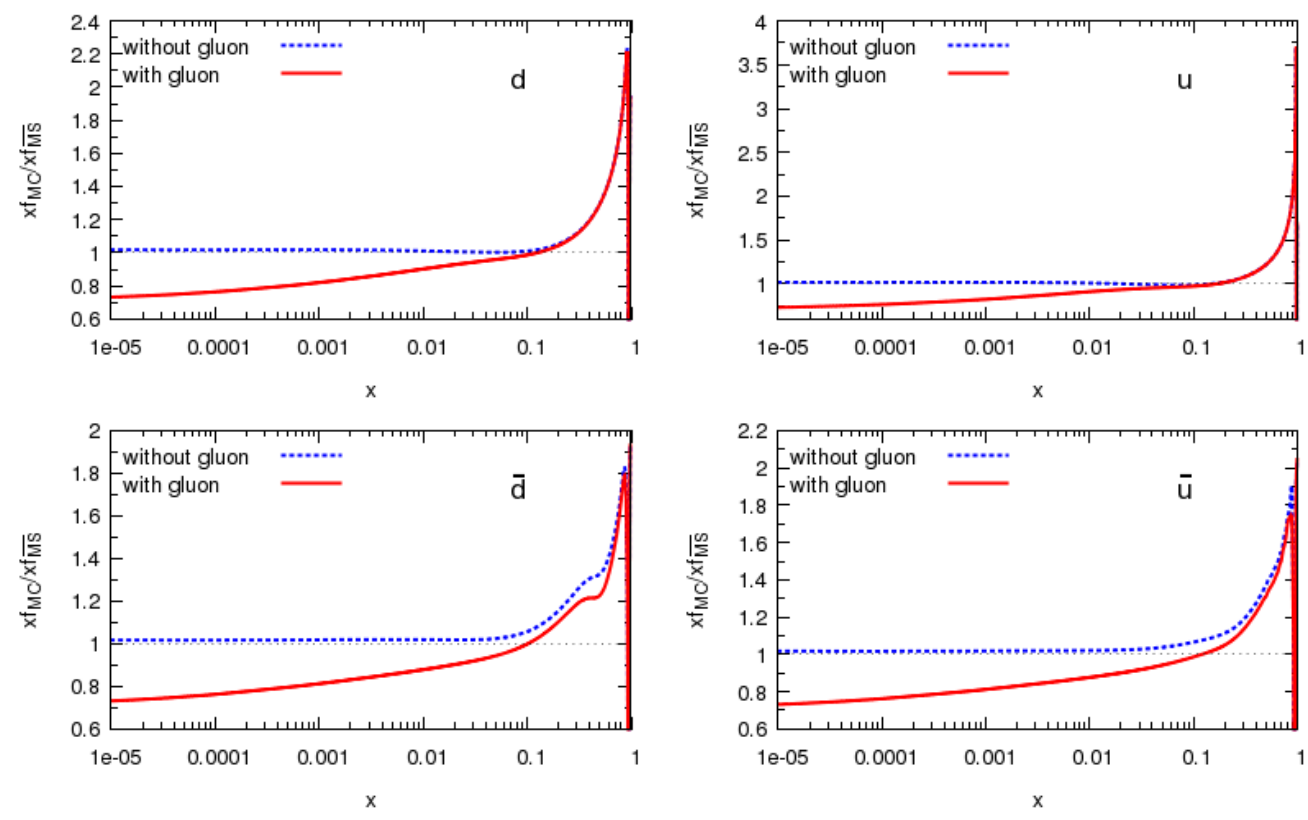

Figure 7. Ratios of MC PDFs to the standard $\overline{\mathrm{MS}}$ PDFs at $Q^{2}=100 \mathrm{GeV}$. The two curves in each plot correspond to the case with (red-solid line) and without (dotted-blue line) the convolution with the gluon distribution in Eq. (3.17).

which results from the fact that the second term on the r.h.s. of eq. (3.17) is always positive. Adding the gluon component to the MC quark makes a little difference at large $x$, whereas the small and moderate $x$ regions are affected significantly. We see that the convolution with $\Delta C_{2 g}$ is negative and, at small $x$, it can reduce the $\overline{\mathrm{MS}}$ quark PDFs even by $20 \%$. We have found a very similar picture for other quark flavors as well for other $Q^{2}$ values.

Before moving to NLO, it is interesting to check how the LO result changes when switching from the $\overline{\mathrm{MS}}$ to MC PDFs. From the LO point of view, both PDFs are equivalent, as the differences are formally of the $\mathcal{O}\left(\alpha_{s}{ }^{2}\right)$ order and higher. We can see, however, some numerical differences in the LO distributions between those cases. And, indeed, as shown in figure 8 , the differential distributions of the dilepton mass and the dilepton rapidity differ at LO depending on whether the $\overline{\mathrm{MS}}$ or the MC PDFs are used. In the case of the MC PDFs without the gluon convolution, cf. eq. (3.17), the mass spectrum is $\sim 5 \%$ higher than that with the $\overline{\mathrm{MS}}$ PDFs over a broad range around the peak. On the other hand, when we include the gluon convolution, the result with MC PDFs gets up to $\sim 20 \%$ below that of $\overline{\mathrm{MS}}$. The dilepton rapidity distribution with the MC PDFs with (without) the gluon is smaller (larger) by a similar amount for central rapidities and grows above $100 \%$ in the forward and backward regions. This can be related to the large $x$ behavior seen in figure 7 via the formula $x_{1,2}=\frac{m_{Z}}{\sqrt{s}} \exp \left( \pm y_{Z}\right)$ valid for $2 \rightarrow 1$ kinematics. Here, $x_{1}$ and $x_{2}$ are the usual hadron's energy fractions of the incoming partons, while $y_{Z}$ is the rapidity of the produced $Z$-boson. The large rapidities in the forward or backward direction correspond to one of the $x$ 'es being large and that is the region where the differences between the $\overline{\mathrm{MS}}$ PDFs and the MC PDFs are substantial. 

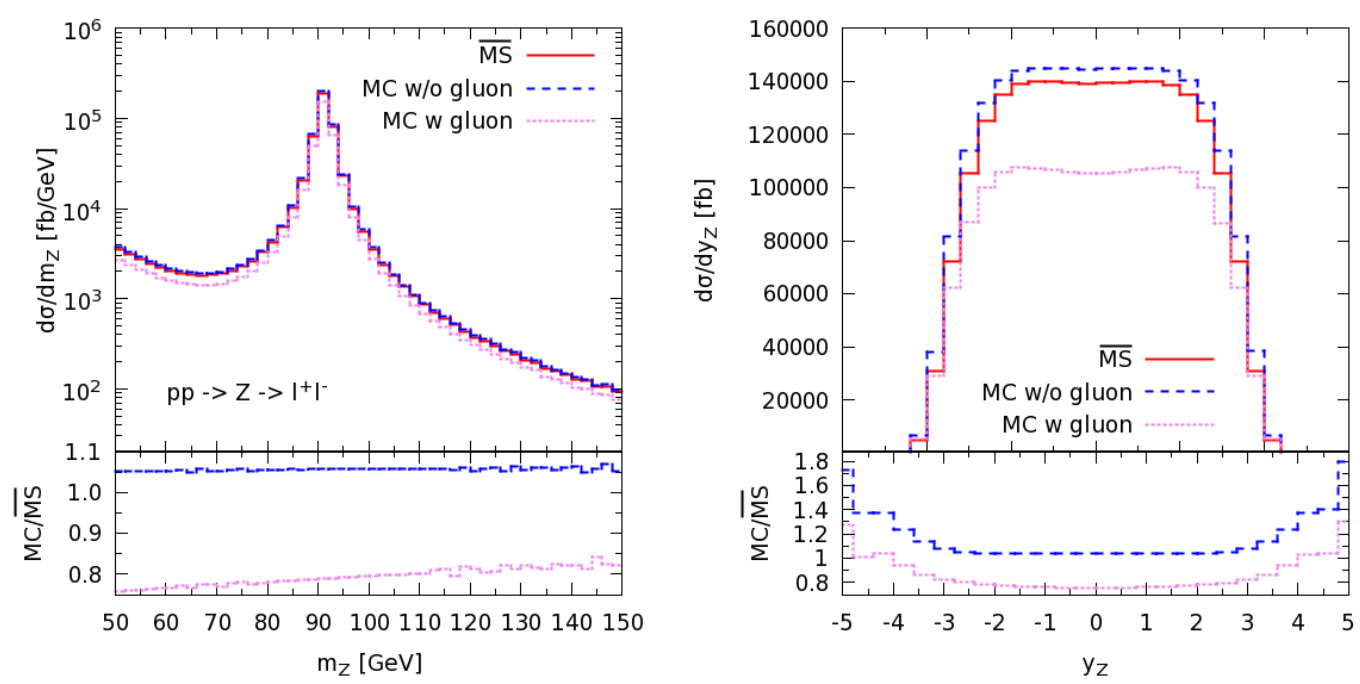

Figure 8. Distributions of the transverse momentum and rapidity from the LO calculation from the MCFM program with the standard $\overline{M S}$ MSTW2008LO PDFS and with PDFs in the MC factorization scale with and without including the gluon convolution in eq. (3.17).

\section{2 $\mathrm{MC}$ and $\overline{\mathrm{MS}}$ schemes at NLO}

The cross section for the Drell-Yan process at NLO in the $\overline{\mathrm{MS}}$ scheme can be schematically written as

$$
\sigma_{\mathrm{DY}}^{\overline{\mathrm{MS}}}=\sum_{\substack{i, j=q, \bar{q} \\ i \neq j}} f_{i}\left(x_{1}\right) \otimes\left[\delta(1-z)+\alpha_{s} \widetilde{C}_{q}^{\overline{\mathrm{MS}}}\right] \otimes f_{j}\left(x_{2}\right)+\sum_{\substack{i=q, \bar{q} ; j=g \\ i=g ; j=q, \bar{q}}} f_{i}\left(x_{1}\right) \otimes \alpha_{s} \widetilde{C}_{g}^{\overline{\mathrm{MS}}} \otimes f_{j}\left(x_{2}\right),
$$

where $f_{i}\left(x_{1,2}\right)$ are the standard $\overline{\mathrm{MS}}$ parton distribution functions, whose scale dependence is understood, and $\otimes$ denotes the convolution via integration over the $z$ variable. The first term in the square brackets is just the Born contribution, which is followed by the NLO correction in the $q \bar{q}$ channel. The second sum corresponds to the NLO contribution from the $q g$ channel. In both cases, the functions $\widetilde{C}_{q, g}^{\overline{\mathrm{MS}}}$ have non-trivial $z$-dependence (suppressed here for clarity of notation) and they are related to the coefficient functions from eqs. (3.11) and (3.14) as $\widetilde{C}_{q, g}^{\overline{\mathrm{MS}}}=\frac{1}{\alpha_{s}} C_{2 q, 2 g}^{\overline{\mathrm{MS}}}(z)$. Hence, the dependence on $\alpha_{s}$ is explicit in eq. (4.3) and all the following formulae of this section.

When going to the MC scheme, the $\overline{\mathrm{MS}}$ coefficient functions in eq. (4.3) need to be replaced by their MC scheme counterparts and the PDFs need to be transferred to the MC scheme as well according to eq. (3.17). This leads to the formula

$$
\begin{aligned}
\sigma_{\mathrm{DY}}^{\mathrm{MC}}= & \sum_{\substack{i, j=q, \bar{q} \\
i \neq j}}\left(f_{i}\left(x_{1}\right)+f_{i}\left(x_{1}\right) \otimes \alpha_{s} \Delta \widetilde{C}_{q}+f_{g}\left(x_{1}\right) \otimes \alpha_{s} \Delta \widetilde{C}_{g}\right) \\
& +\sum_{i=q, \bar{q}}\left(f_{i}\left(x_{1}\right)+f_{i}\left(x_{1}\right) \otimes \alpha_{s} \Delta \widetilde{C}_{q}+f_{g}\left(x_{1}\right) \otimes \alpha_{s} \Delta \widetilde{C}_{g}\right) \otimes \alpha_{s} \widetilde{C}_{g}^{\mathrm{MC}} \otimes f_{g}\left(x_{2}\right) \\
& +\sum_{j=q, \bar{q}} f_{g}\left(x_{1}\right) \otimes \alpha_{s} \widetilde{C}_{g}^{\mathrm{MC}} \otimes\left(f_{j}\left(x_{2}\right)+f_{j}\left(x_{2}\right) \otimes \alpha_{s} \Delta \widetilde{C}_{q}+f_{g}\left(x_{2}\right) \otimes \alpha_{s} \Delta \widetilde{C}_{g}\right),
\end{aligned}
$$




\begin{tabular}{|c|c|c|c|}
\hline \multicolumn{4}{|c|}{$\mathrm{LO}$} \\
\hline \multicolumn{2}{|c|}{$\sigma_{\mathrm{DY}}^{(0)}[\mathrm{pb}] q \bar{q}$ channel } & \multicolumn{2}{|c|}{$\sigma_{\mathrm{DY}}^{(0)}[\mathrm{pb}]$ both channels } \\
\hline$\overline{\mathrm{MS}}$ & $936.79 \pm 0.30$ & $\overline{\mathrm{MS}}$ & $988.9 \pm 0.30$ \\
\hline $\begin{array}{c}\mathrm{MC} \\
\text { in which: }\end{array}$ & $989.18 \pm 0.32$ & $\begin{array}{c}\mathrm{MC} \\
\text { in which: }\end{array}$ & $778.8 \pm 0.20$ \\
\hline \multicolumn{4}{|c|}{$\mathcal{O}\left(\alpha_{s}\right)$} \\
\hline$f_{q} f_{\bar{q}}$ & $25.79 \pm 0.04$ & $f_{q} f_{\bar{q}}+f_{\bar{q}} f_{q}$ & $54.8 \pm 0.4$ \\
\hline$f_{\bar{q}} f_{q}$ & $25.79 \pm 0.02$ & $f_{q} f_{g}+f_{\bar{q}} f_{g}$ & $-271.4 \pm 0.4$ \\
\hline \multicolumn{4}{|c|}{$\mathcal{O}\left(\alpha_{s}^{2}\right)$} \\
\hline & $0.64 \pm 0.01$ & & $6.70 \pm 0.20$ \\
\hline
\end{tabular}

Table 2. Values of the total cross section with statistical errors for the the Drell-Yan process at LO in $\overline{\mathrm{MS}}$ and MC factorization schemes. The results were obtained with MCFM 6.6 [32]. The $\mathcal{O}\left(\alpha_{s}\right)$ admixture in the MC results is split into contributions proportional to various terms of eq. (4.4).

where, again $\widetilde{C}_{q, g}^{\mathrm{MC}}=\frac{1}{\alpha_{s}} C_{2 q, 2 g}^{\mathrm{MC}}(z)$, with the $\mathrm{MC}$ coefficient functions defined in eqs. (3.12) and (3.15), and similarly for $\Delta \widetilde{C}_{q, g}=\frac{1}{\alpha_{s}} \Delta C_{2 q, 2 g}(z)$, with the latter given in eqs. (3.13) and (3.16).

By construction (cf. section 3.2), terms proportional to a given partonic luminosity, $f_{i} f_{j}$, are identical in both factorization schemes up to the order $\mathcal{O}\left(\alpha_{s}\right)$. The results in eqs. (4.3) and (4.4) differ however at $\mathcal{O}\left(\alpha_{s}^{2}\right)$, which is beyond NLO and therefore such a difference is allowed.

In order to validate our transformation from the $\overline{\mathrm{MS}}$ to the $\mathrm{MC}$ scheme, we have performed an explicit check of the equivalence of the two schemes up to $\mathcal{O}\left(\alpha_{s}\right)$, as well studied numerical importance of the higher order terms. The calculations were performed using our standard setup defined at the beginning of section 4 .

Let us start from comparing the cross sections at LO. This corresponds to setting the coefficient functions $\widetilde{C}_{q, g}=0$ in eqs. (4.3) and (4.4). As shown in the previous section, the LO cross sections will differ between the two schemes because of the PDFs. To check the extent to which this happens, we performed an explicit computation with MCFM [32] in both factorization schemes for either the pure $q \bar{q}$ channel or both channels.

Indeed, as demonstrated in table 2 , the $\mathrm{LO} \overline{\mathrm{MS}}$ and $\mathrm{MC}$ cross sections are not identical, both in the case of the pure $q \bar{q}$ channel, where the LO result with MC PDFs is $\sim 5 \%$ higher, and in the case where both channels are included, where the MC result is $\sim 20 \%$ lower. The beyond-LO, $\mathcal{O}\left(\alpha_{s}\right)$ and $\mathcal{O}\left(\alpha_{s}^{2}\right)$, terms are also given in the table. We see that most of the difference comes from $\mathcal{O}\left(\alpha_{s}\right)$. The $\mathcal{O}\left(\alpha_{s}{ }^{2}\right)$ terms are in fact very small, below $1 \%$ in both cases. It is interesting to note that the difference between $\sigma_{\mathrm{DY}}^{(0)}$ in the two schemes, for the case with both channels, comes primarily from the term proportional to $\alpha_{s} f_{q} \otimes \Delta C_{g} \otimes f_{g}$, hence it originates from the large gluon luminosity. 


\begin{tabular}{|c|c|c|c|}
\hline \multicolumn{4}{|c|}{ NLO } \\
\hline \multicolumn{2}{|c|}{$\sigma_{\mathrm{DY}}^{(1)}[\mathrm{pb}] q \bar{q}$ channel } & \multicolumn{2}{|c|}{$\sigma_{\mathrm{DY}}^{(1)}[\mathrm{pb}]$ both channels } \\
\hline$\overline{\mathrm{MS}}$ & $336.36 \pm 0.09$ & $\overline{\mathrm{MS}}$ & $157.9 \pm 0.10$ \\
\hline $\begin{array}{c}\mathrm{MC} \\
\text { in which: }\end{array}$ & $352.96 \pm 0.09$ & $\begin{array}{c}\mathrm{MC} \\
\text { in which: }\end{array}$ & $305.8 \pm 0.10$ \\
\hline \multicolumn{4}{|c|}{$\mathcal{O}\left(\alpha_{s}\right)$} \\
\hline$f_{q} \Delta \widetilde{C}_{q} f_{\bar{q}}$ & $25.79 \pm 0.04$ & $f_{q} \Delta \widetilde{C}_{q} f_{\bar{q}}+f_{\bar{q}} \Delta \widetilde{C} f_{q}$ & $355.00 \pm 0.29$ \\
\hline$f_{\bar{q}} \Delta \widetilde{C}_{q} f_{q}$ & $25.79 \pm 0.02$ & $f_{q} \widetilde{C}_{q}^{\mathrm{MC}} f_{\bar{q}}+f_{\bar{q}} \widetilde{C}_{q}^{\mathrm{MC}} f_{q}$ & $-17.10 \pm 0.14$ \\
\hline$f_{q, \bar{q}} \widetilde{C}_{q}^{\mathrm{MC}} f_{\bar{q}, q}$ & $284.77 \pm 0.08$ & $f_{q} \widetilde{C}_{g}^{\mathrm{MC}} f_{g}+f_{\bar{q}} \widetilde{C}_{g}^{\mathrm{MC}} f_{g}$ & $-180.10 \pm 0.10$ \\
\hline sum & $336.35 \pm 0.09$ & sum & $157.80 \pm 0.34$ \\
\hline \multicolumn{4}{|c|}{$\mathcal{O}\left(\alpha_{s}^{2}\right)+\mathcal{O}\left(\alpha_{s}^{3}\right)$} \\
\hline $\mathrm{MC}$ & $16.61 \pm 0.02$ & $\mathrm{MC}$ & $147.9 \pm 0.20$ \\
\hline
\end{tabular}

Table 3. Values of the NLO contribution to the total cross section with statistical errors for the the Drell-Yan process in the $\overline{\mathrm{MS}}$ and MC factorization schemes. The results were obtained with MCFM 6.6 [32] and its version adjusted to the MC scheme, MCFM*. The $\mathcal{O}\left(\alpha_{s}\right)$ corrections in the $\mathrm{MC}$ results is split into contributions proportional to various terms of eq. (4.4).

We turn now to a similar comparison at NLO. Table 3 gives the NLO-only results in the two schemes for the cases of $q \bar{q}$ and both channels. At NLO, we need to be careful what we take for the coefficient functions. In the $\overline{\mathrm{MS}}$ scheme, we just use the original MCFM 6.6 [32] implementation. However, in order to carry out consistent NLO calculations in the MC scheme, we had to change the coefficient functions from $C_{2 q, 2 g}^{\overline{\mathrm{MS}}}(z)$ to $C_{2 q, 2 g}^{\mathrm{MC}}(z)$. We dubbed this modified version of the program MCFM* and used it together with the MC PDFs to compute our NLO predictions in that scheme.

We see that also the subleading corrections to the Drell-Yan process are not the same in the $\mathrm{MC}$ and $\overline{\mathrm{MS}}$ schemes (numbers in the two first lines of table 3). This is allowed provided that all the difference comes from terms of the order $\alpha_{s}^{2}$ and beyond. As shown in table 3, by extracting only the $\mathcal{O}\left(\alpha_{s}\right)$ terms of the MC result and summing them up, we recover the $\overline{\mathrm{MS}}$ cross section exactly. There, we also give subleading correction introduced via the MC PDFs and we see that they contribute at most $5 \%$ to the NLO correction in the MC scheme in the case of the pure $q \bar{q}$ channel but can be quite sizable when both channels are considered.

Finally, it would be interesting to fit the quark and gluon PDFs in the MC factorization scheme directly to experimental data, in order to minimize higher-order effects. For that we would need to define the gluon PDF in the MC factorization scheme using a process in which the gluon PDF enters already at the LO level, e.g. the Higgs-boson production process. This will be done in our future publication. 
Let us conclude that we have successfully validated the MC factorization scheme by explicitly showing that all numerical differences w.r.t. the $\overline{\mathrm{MS}}$ results are of the order of $\alpha_{s}{ }^{2}$ and $\alpha_{s}{ }^{3}$. We emphasize that the validation of the new MC scheme presented in this section provides a highly non-trivial check as various components, given schematically in eq. (3.17), come from different parts of the calculation (PDFs, coefficient functions) and the agreement up to $\alpha_{s}$ is much more sophisticated than a purely algebraic relation.

\section{Results for NLO with parton shower}

Numerical implementation of KrkNLO is presently done mainly using the Sherpa PSMC of ref. [11] version 2.0.0 $0^{29}$ with the dipole organization of the parton shower distributions inspired by the Catani-Seymour work [24], see ref. [40]. However, the evolution variable in ref. [40] is chosen to be the transverse momentum distribution, while in the actual Sherpa 2.0.0 implementation it is the $q^{2} \sim \alpha(\alpha+\beta)$ variable of section 2, see ref. [41]. The dipole shower implemented in Herwig ++ , see ref. [23], is quite similar. Actually, the choice of the evolution variable is not critical in the KrkNLO method, as long as the full coverage of the phase space for the emission closest to the hard process is assured, and the PSMC distribution is under the perfect control. In fact, for any choices of the dipole PS evolution variable in Sherpa or Herwig ++ the same distribution of the single-gluon emission is obtained, provided that the contributions for the quark and antiquark emitters are added. For the Sherpa MC program, we have checked numerically the completeness of the coverage of the phase space of the first emission from the backward evolution by examining the gluon distribution on the $\alpha, \beta$ plane.

In the following, the KrkNLO-matched results will be compared mostly with the results of the MCONLO technique, implemented on top of the same parton shower within Sherpa. ${ }^{30}$ Using the same parton shower in both cases ensures that all differences in the matched results come purely from the differences in the methods themselves rather than details of the shower implementation. Nevertheless, the difference between the dipole showers currently implemented in Sherpa and Herwig++ turns out to be small. From the point of view of our study, it amounts mainly to using the $q$ variable in the former and $k_{T}$ in the latter. These two evolution variables are, however, very close to each other in practice. Hence, in our final discussion, we shall also compare the KrkNLO results with those of POWHEG, as implemented in Herwig $++{ }^{31}$ using an automated setup based on the Matchbox framework [42] with adaptive sampling [43].

In the numerical validation of the KrkNLO approach, we shall first compare it to the fixed-order NLO calculation, as implemented in the MCFM program. Later on, the comparison with the MCQNLO implementation of Sherpa will be discussed. In order to minimize the influence of various higher-order effects on the difference between KrkNLO and MCFM, we shall initially limit our numerical exercises to a simplified version, in which

\footnotetext{
${ }^{29}$ https://sherpa.hepforge.org/doc/SHERPA-MC-2.0.0.html.

${ }^{30}$ Unfortunately, the POWHEG method is not available in Sherpa 2.0.0 framework.

${ }^{31}$ For our comparisons we used the version of POWHEG without restrictions of the phase space, which is similar to the original POWHEG method described in ref. [3].
} 
only the $q \bar{q}$ channel is kept. Another initial limitation, with the same aim in mind, will be to profit from the option in Sherpa to stop the backward evolution in the parton shower just after the first emission (starting from the hard process). This will help us to eliminate the influence of the differences in the so-called recoil schemes [44], which start to play a role from the second emission onwards. The running $\alpha_{s}$ will be also introduced gradually. Since we study the differences in the matching methods, we have switched off in Sherpa nonperturbative effects including the intrinsic $k_{T}$, multiparton interactions and hadronization. However, we would like to stress that there is no problem with switching them on for the KrkNLO method.

In all the following results for the KrkNLO method the PDFs in the MC factorization scheme, discussed in section 4.1 , will be used. The factorization scale will be set to $\mu_{F}=$ $M_{Z}$. As for the choice of the renormalization scale in the matched NLO + PS results, we note that there is always some level of arbitrariness. In the pure fixed-order NLO calculation, in the case of DY, one usually sets $\mu_{R}=\mu_{F}=M_{Z}$. On the other hand, the LO parton shower uses $\mu_{R}^{2}=q^{2}$, with the latter being closely related to the evolution variable of the shower. The parton shower is unitary, hence the choice of the argument of $\alpha_{s}\left(\mu_{R}^{2}\right)$ does not influence the total cross section, it changes, however, shapes of some distributions, e.g. of $p_{T, Z}$. The differences between $\alpha_{s}\left(q^{2}\right)$ and $\alpha_{s}\left(M_{Z}^{2}\right)$ are formally of the higher order, that is beyond NLO and, from that perspective, they are equivalent in the context of NLO + PS matching. They can, however, be numerically relevant.

In what follows, for the KrkNLO method we adopt the following procedure. The argument of $\alpha_{s}$ in the virtual correction is always set to $M_{Z}^{2}$. For the real correction, all emissions except for the first one (in BEV) are set by the parton shower according to its current $q^{2}$ value. For the first emissions, we consider two choices: $q^{2}$ and $M_{Z}^{2}$, both in the real correction and in the Sudakov form factor, to keep the shower unitary. The difference between the results corresponding to these two choices will be indicative of the size of beyond-NLO terms.

\subsection{Initial results for $q \bar{q}$ channel only}

The first round of numerical tests of KrkNLO focuses on comparisons with MCFM and MC@NLO for the $q \bar{q}$ channel only and stopping the parton shower after the first emission. The corresponding results for the total cross section are presented in table 4 and for the transverse momentum and rapidity distributions in figure 9. As we can see, the total cross section and the rapidity distributions from KrkNLO agree very well with those of MCFM and MC@NLO. The difference between the KrkNLO results and the pure fixed-order ones is below $1 \%$ and comes from the $\mathcal{O}\left(\alpha_{s}^{2}\right)$ contamination due to the MC PDFs, cf. eq. (4.4). Also, the differences between the $\alpha_{s}\left(q^{2}\right)$ and $\alpha_{s}\left(M_{Z}^{2}\right)$ are at the per-mille level.

On the other hand, there are remarkable differences in the $p_{T, Z}$ distributions, especially at the lower end. They compensate in the total cross section between the first and the following bins. However, the choice of the running or non-running $\alpha_{s}$ is numerically important for the $p_{T}$ distribution at small and moderate values. The perfect agreement of MC@NLO and MCFM at higher $p_{T}$ is of course enforced by construction. This is not the case in KrkNLO, where some part of higher-order effects, beyond NLO, is included (which 


\begin{tabular}{|l|c|}
\hline & $\sigma_{\text {tot }}^{q \bar{q}}[\mathrm{pb}]$ \\
\hline \hline MCFM & $1273.4 \pm 0.1$ \\
MC@NLO & $1273.4 \pm 0.1$ \\
POWHEG & $1272.1 \pm 0.7$ \\
\hline KrkNLO $\alpha_{s}\left(q^{2}\right)$ & $1282.6 \pm 0.2$ \\
KrkNLO $\alpha_{s}\left(M_{Z}^{2}\right)$ & $1285.3 \pm 0.2$ \\
\hline
\end{tabular}

Table 4. Values of the total cross section with statistical errors for the Drell-Yan process (the $q \bar{q}$ channel only) from the KrkNLO method compared to the fixed-order result of MCFM and the results of MCONLO and POWHEG.

$8 \mathrm{TeV}: q \bar{q}$ channel (1st emission only)

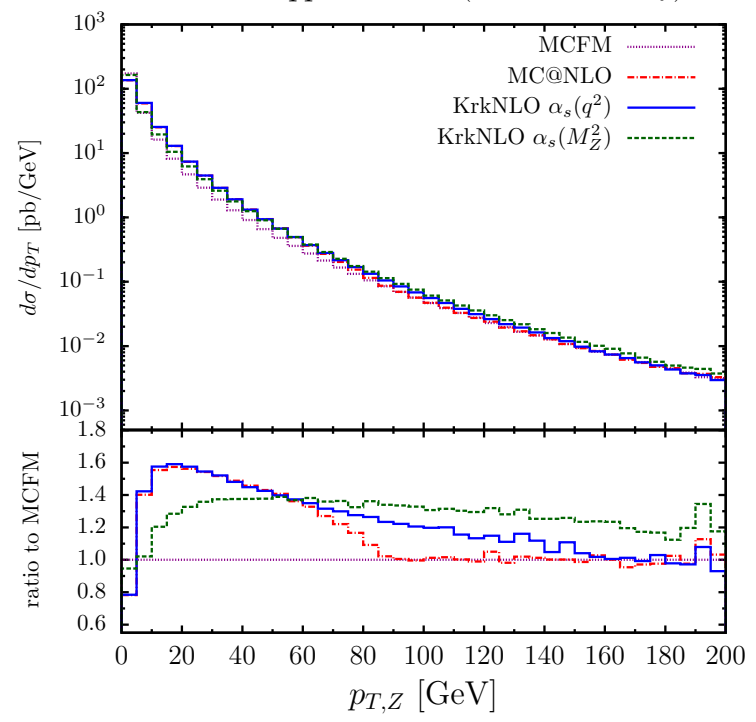

$8 \mathrm{TeV}: q \bar{q}$ channel (1st emission only)

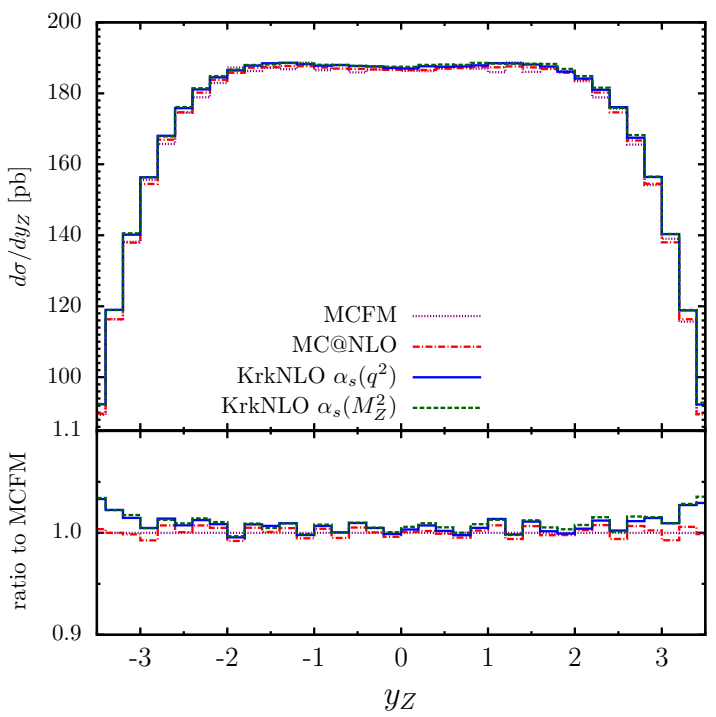

Figure 9. Comparisons of the transverse momentum and rapidity distributions from MCFM, MC@NLO and two versions of KrkNLO, one with fixed $\alpha_{s}\left(M_{z}^{2}\right)$ (also in MCFM) and another one with running $\alpha_{s}$ depending on the transverse momentum squared. KrkNLO method is implemented within Sherpa parton shower MC. The exercise is restricted to the $q \bar{q}$ channel only and the parton shower backward evolution is stopped after the first emission, the closest to the hard process.

is also the case in POWHEG). We see, however, that both MC@NLO and KrkNLO coincide below $\sim 80 \mathrm{GeV}$ in the case with $\alpha_{s}\left(q^{2}\right)$.

In the results of figure 9 the parton shower was artificially stopped after the first emission, in order to make the meaningful comparisons with MCFM and limit the effects of the recoil due to subsequent emissions. In figure 10 we lift this limitation and the parton shower goes to the very end, as in the normal operational mode of PSMC. As we see, switching on to the full PS influences considerably the low $p_{T}$ part of the spectrum, in spite of a negligible effect on the rapidity distribution (and hence, on the total cross section).

Again, KrkNLO with the running $\alpha_{s}$ agrees very well with MC@NLO at low and moderate $p_{T}$, which results from the domination of the parton shower contribution in that 
$8 \mathrm{TeV}: q \bar{q}$ channel (full parton shower)

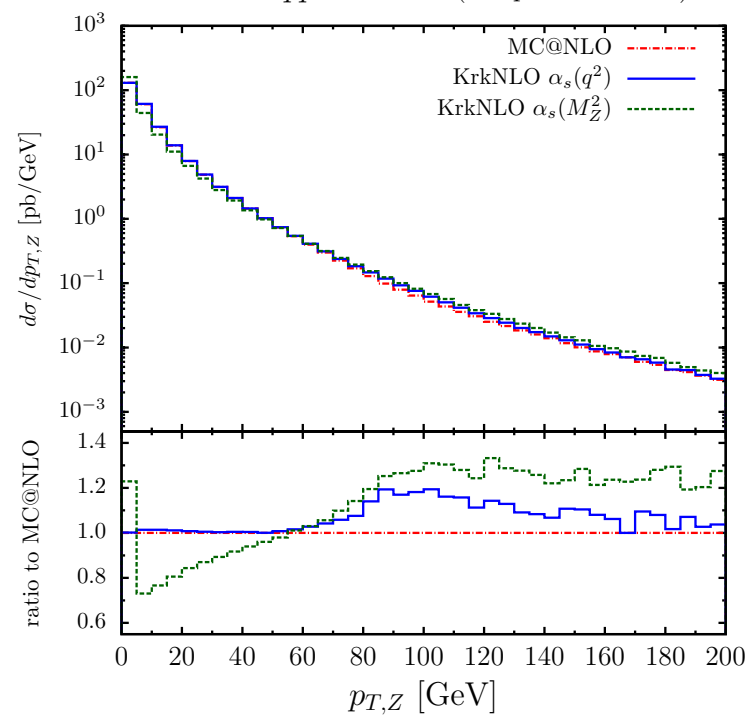

$8 \mathrm{TeV}: q \bar{q}$ channel (full parton shower)

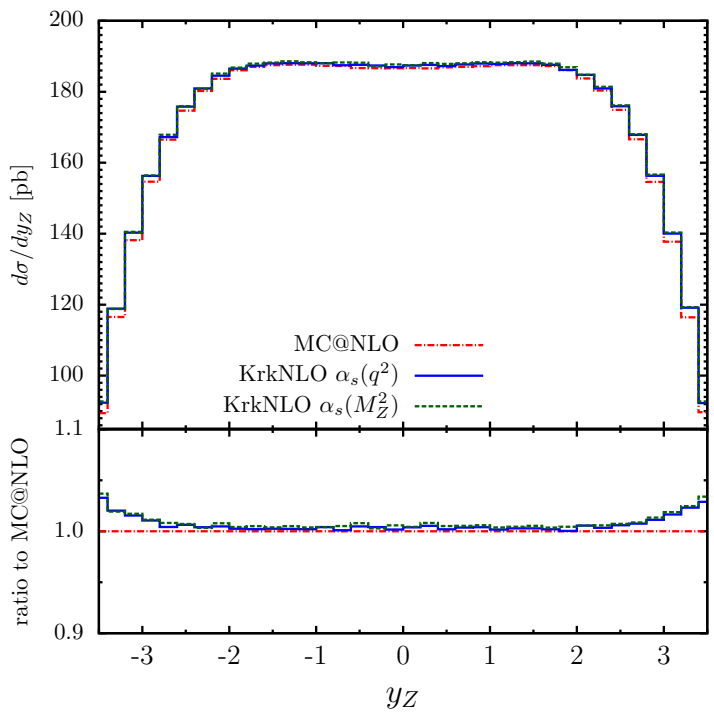

Figure 10. Comparisons of the transverse momentum and rapidity distributions from MCQNLO and two versions of KrkNLO for the $q \bar{q}$ channel only as in figure 9, but the parton shower backward evolution runs to the end as normally.

region. However, the two KrkNLO results stay above MC@NLO at higher $p_{T}$, which we again attribute to the admixture of some NNLO terms in the former. The difference between $\operatorname{KrkNLO} \alpha_{s}\left(q^{2}\right)$ and KrkNLO $\alpha_{s}\left(M_{Z}^{2}\right)$ at high $p_{T}$ comes purely from the running of the coupling.

\subsection{All channels}

In the second round of the numerical tests, all the initial-state parton combinations contributing at NLO, that is the $q \bar{q}$ and $q g$ channels are included. The total cross sections from KrkNLO, MCFM and MCONLO are compared in table 5. As we can see, the difference between MCFM/MC@NLO and KrkNLO, which comes from the partial inclusion of the higher-order effects in the latter, is about twice as big as in the $q \bar{q}$ channel only. As discussed in section 4.2 , this is related to the large gluon luminosity which leads to sizable differences between the MC and $\overline{M S}$ PDFs, and part of that difference shows up in the total cross section. The results in table 5 are generally consistent with the differences staying below $5 \%$.

In figure 11 we show the distributions of the transverse momentum and rapidity of the $Z$-boson with the parton shower stopped after the first emission. The lower panel contains the ratio with respect to MCFM. Similarly to the case of the pure $q \bar{q}$ channel, we see the expected agreement between MCONLO and MCFM at large $p_{T, Z}$. The KrkNLO results with the fixed $\alpha_{s}$ are not far from the MCFM ones in that region as well. On the other hand, all the PS-matched results differ from those of MCFM at smaller $p_{T}$, because of the lack of the Sudakov resummation in MCFM, see below for more discussion on this issue. Each curve behaves slightly differently but the variations are moderate. The rapidity distributions shown in figure 11 (right) are close to each other for all calculations. The $\sim 5 \%$ difference between KrkNLO and MCFM is the same as for the total cross sections 


\begin{tabular}{|l|c|}
\hline & $\sigma_{\text {tot }}^{q \bar{q}+q g}[\mathrm{pb}]$ \\
\hline \hline MCFM & $1086.5 \pm 0.1$ \\
MC@NLO & $1086.5 \pm 0.1$ \\
POWHEG & $1084.2 \pm 0.6$ \\
\hline KrkNLO $\alpha_{s}\left(q^{2}\right)$ & $1045.4 \pm 0.1$ \\
KrkNLO $\alpha_{s}\left(M_{Z}^{2}\right)$ & $1039.0 \pm 0.1$ \\
\hline
\end{tabular}

Table 5. Values of the total cross section with statistical errors for the Drell-Yan process, both channels, from the KrkNLO method compared to the fixed-order result of MCFM and the results of POWHEG and MC@NLO.

$8 \mathrm{TeV}: q \bar{q}$ and $q g$ channels (1st emission only)

$8 \mathrm{TeV}: q \bar{q}$ and $q g$ channels (1st emission only)
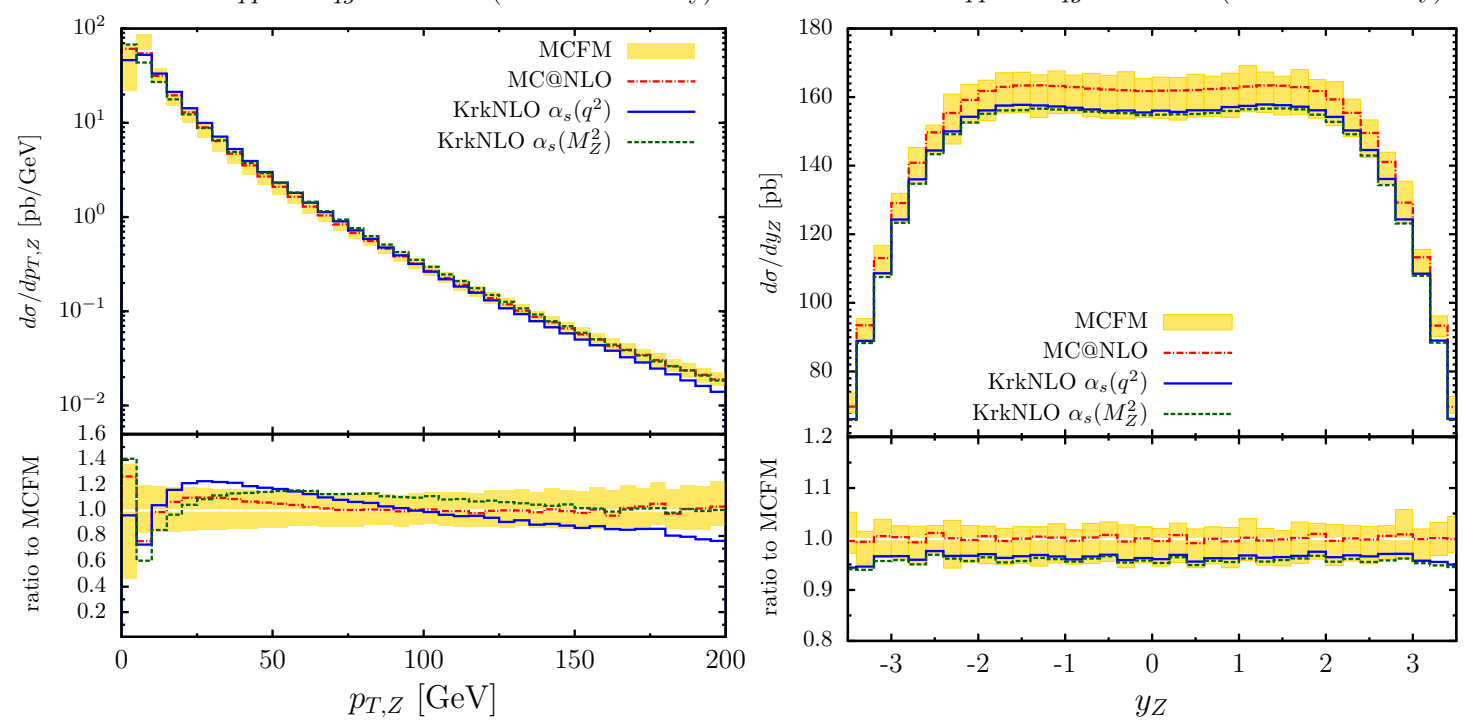

Figure 11. Comparisons of the transverse momentum and rapidity distributions from MCFM, MCQNLO and two versions of KrkNLO, now for both the $q \bar{q}$ and $q g$ channels, with $\alpha_{s}$ set as in the previous figures and the parton shower backward evolution stopped after the first emission as in figure 9 .

in table 5. In figure 11 we have also included the scale-uncertainty bands, which were produced using MCFM by means of changing (independently) both the factorization and the renormalization scales by the customary factors of 2 and $1 / 2$. The differences between MCFM and KrkNLO are enveloped by these bands.

Figure 12 shows the similar distributions but, this time, the parton shower is allowed for an arbitrary number of emissions.

There, we also show the results from POWHEG, as implemented in Herwig++. Even though the evolution variable is different $\left(k_{T}\right.$ rather than $q$ of eq. (2.7) in all the other PS-matched results), POWHEG agrees quite well with the KrkNLO $\alpha_{s}\left(q^{2}\right)$ version. This shows that the choice of the evolution variable in PS between $k_{T}$ and $q$ is not so important for numerical results. The distributions of the transverse momentum and rapidity look 

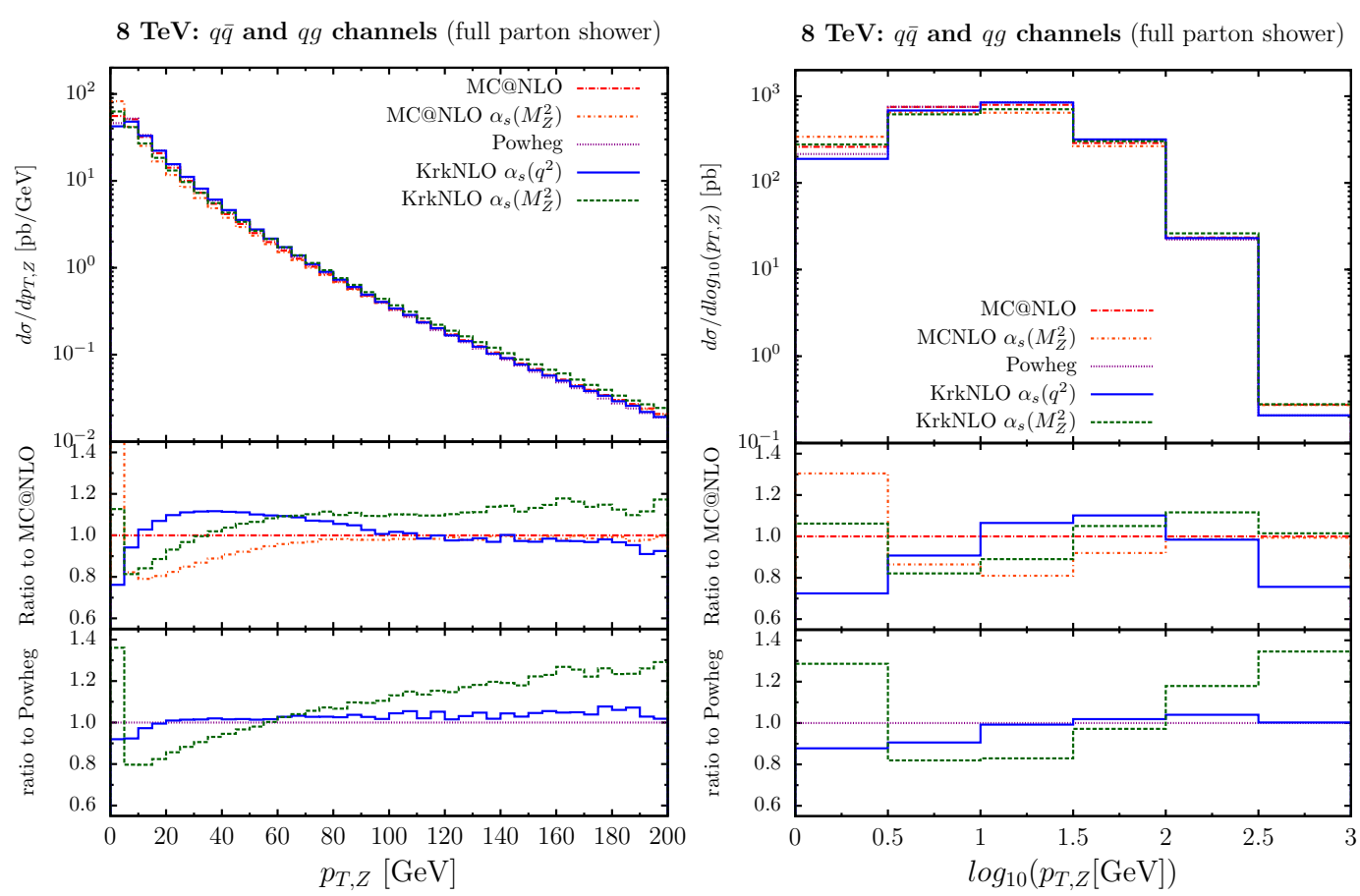

Figure 12. Comparisons of the transverse momentum distribution from MC@NLO, POWHEG and two versions of KrkNLO for both the $q \bar{q}$ and $q g$ channels as in figure 11, but the parton shower backward evolution runs to the end as normally.

quite similar to the previous results for the pure $q \bar{q}$ channel in figure 10. The transverse momentum distribution from KrkNLO agrees with MC@NLO at high $p_{T, Z}$ (up to a constant factor in the case with the fixed $\alpha_{s}$ ), while larger differences are seen in the low $p_{T}$ region.

The differences of the order of $10-20 \%$ seen at low $p_{T}$ between MCFM, MC@NLO, POWHEG and KrkNLO in figures 10, 11 and 12 reflect mainly the parton-shower feature of the soft-gluon resummation in the case of the matched results and the lack of it in the case of the fixed-order NLO calculations. More precisely, parton shower (without intrinsic $k_{T}$ ) has a gap below the minimum $p_{T}$ ending the backward evolution and a non-physical spike $\sim \delta\left(p_{T}\right)$. Normalization of the spike is governed by unitarization enforced by the shower. Kinematics, histogramming, intrinsic $k_{T}$, etc. are smearing the shape of this nonphysical structure. Different implementations of the argument of $\alpha_{s}$ strongly influence this structure near $p_{T}=0$ and may show up in the ratios of the $p_{T}$ distribution as large effects there. In particular, our results with $\alpha_{s}\left(M_{Z}^{2}\right)$ at low $p_{T}$ are very different from the results with $\alpha_{s}\left(q^{2}\right)$. The latter choice better represents resummation of subclasses of higher-order QCD contributions, relevant at low $p_{T}$, and it is therefore commonly adopted in PS MCs. In figure 12 another curve is added for MC@NLO with $\alpha_{s}\left(M_{Z}^{2}\right)$, demonstrating that the same kind of spike and dip near $p_{T}=0$ is present in the corresponding ratios, even for the MC@NLO alone. Hence we conclude that the numerical results from KrkNLO near $p_{T}=0$ are well understood and look as expected.

Even better agreement is found in the comparison of results from $\operatorname{KrkNLO} \alpha_{s}\left(q^{2}\right)$ and POWHEG. Here, the two $p_{T, Z}$ distributions almost coincide in the whole range shown in 
figure 12 (left bottom panel). This is related to a similar, multiplicative, way of applying the NLO correction to the parton shower in KrkNLO and POWHEG. In both cases, this corresponds to the virtual part of the NLO correction being applied over the entire $p_{T, Z}$ range. This is different in MC@NLO, where the virtual correction is spread by the shower only up to the upper limit of shower's evolution variable (starting scale for BEV shower). That upper limit is typically set at the scale of a vector boson mass. Hence, in MCONLO, the $p_{T, Z}$ distribution in the region $p_{T, Z}>m_{Z}$ is corrected only with the real part of NLO, and therefore it recovers exactly the fixed order NLO result in this region. The multiplicative nature of the KrkNLO and POWHEG methods leads to mixed real-virtual corrections of the order $\alpha_{s}^{2}$ at high $p_{T, Z}$. These corrections are however part of NNLO, hence they go beyond the accuracy of NLO+PS result.

The rapidity distributions shown in figure 13 agree very well in the central region between all the PS-matched results, up to the normalization differences, the same as in table 5. Differences at forward and backward rapidities come from increasing differences between PDFs in MC and $\overline{\mathrm{MS}}$ schemes as $x \rightarrow 1$, cf. figure 7 .

Finally, in figure 14 (left) we compare the KrkNLO results for the $Z$-boson transverse momentum distribution, from both channels and with full PS, with the corresponding result obtained from the fixed-order NNLO calculations implemented in the DYNNLO program [46]. The NNLO distributions were obtained with the NNLO MSTW2008 PDFs and using $\mu_{F}=\mu_{R}=m_{Z}$, varied by the customary factors of $1 / 2$ and 2 to estimate the uncertainty from neglected higher orders. Similar comparisons between the NNLO results and those of POWHEG and MC@NLO are shown in figure 14 (right). All the results are divided by the NLO distribution from MCFM.

The calculations within the KrkNLO method were performed with two choices of the argument of the strong coupling $\alpha_{s}: q^{2}$ and $M_{Z}^{2}$, as was done earlier in the paper. These results are shown in figure 14 (left) as a solid blue line and dashed green line, respectively. In addition, in solid orange, we show the result of the KrkNLO matching in the case where the argument of $\alpha_{s}$ is set to the minimum of $q^{2}$ and $M_{Z}^{2}$. This is a physically motivated choice as at high $p_{T, Z}$ our matched result should be driven by the fixed-order component, whose natural scale is $M_{Z}$.

As we see in figure 14 (left), both the KrkNLO and the NNLO results show the same trends, quickly raising above the NLO result at low and moderate $p_{T, Z}$ and then staying above it at high $p_{T, Z}$ (the curve for the case with $\alpha_{s}\left(q^{2}\right)$ falls down due to the fact that the strong coupling is running even at high $p_{T, Z}$ ). The fact that our method gives the result that is higher than the NLO one at high $p_{T, Z}$ is a consequence of the mixed real-virtual $\mathcal{O}\left(\alpha_{s}^{2}\right)$ terms, which constitute part of the NNLO correction and arise because of the multiplicative nature of the KrkNLO approach. Especially the $\alpha_{s}\left(\min \left(q^{2}, m_{Z}^{2}\right)\right)$ choice does a good job, reproducing very closely the full NNLO distribution. Therefore this argument of the strong coupling is likely to be adopted in the future phenomenological studies.

In figure 14 (right) we show similar comparisons with NNLO for MC@NLO and POWHEG. The behavior at low $p_{T, Z}$ is close to that from KrkNLO. At high $p_{T, Z}$, however, MC@NLO and POWHEG converge by construction to the NLO results, departing from the NNLO predictions. 


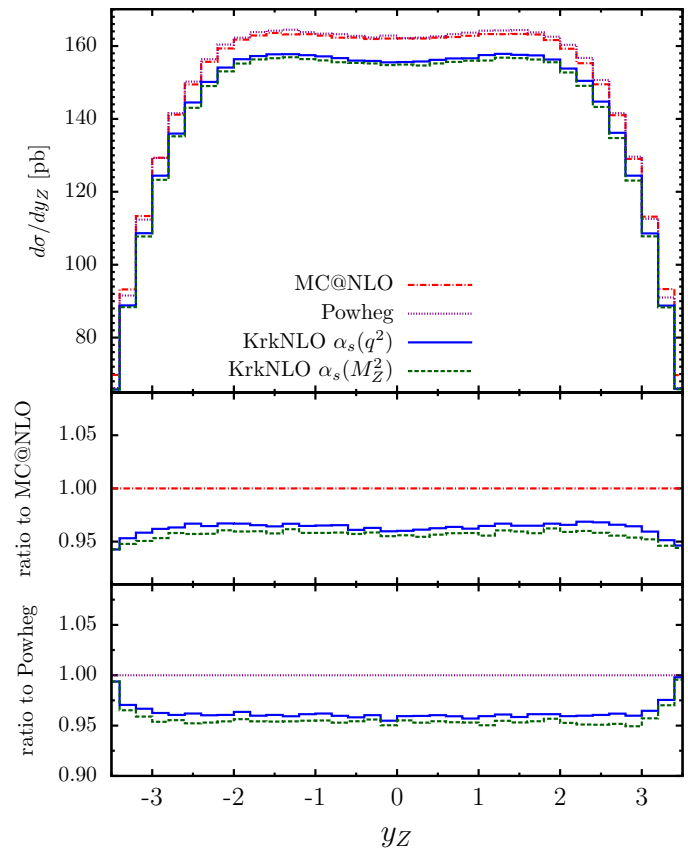

Figure 13. Comparison of the rapidity distribution from MCFM, MC@NLO and two versions of KrkNLO for $q \bar{q}$ and $q g$ channels as in figure 11, but parton shower backward evolution runs to the end as normally.
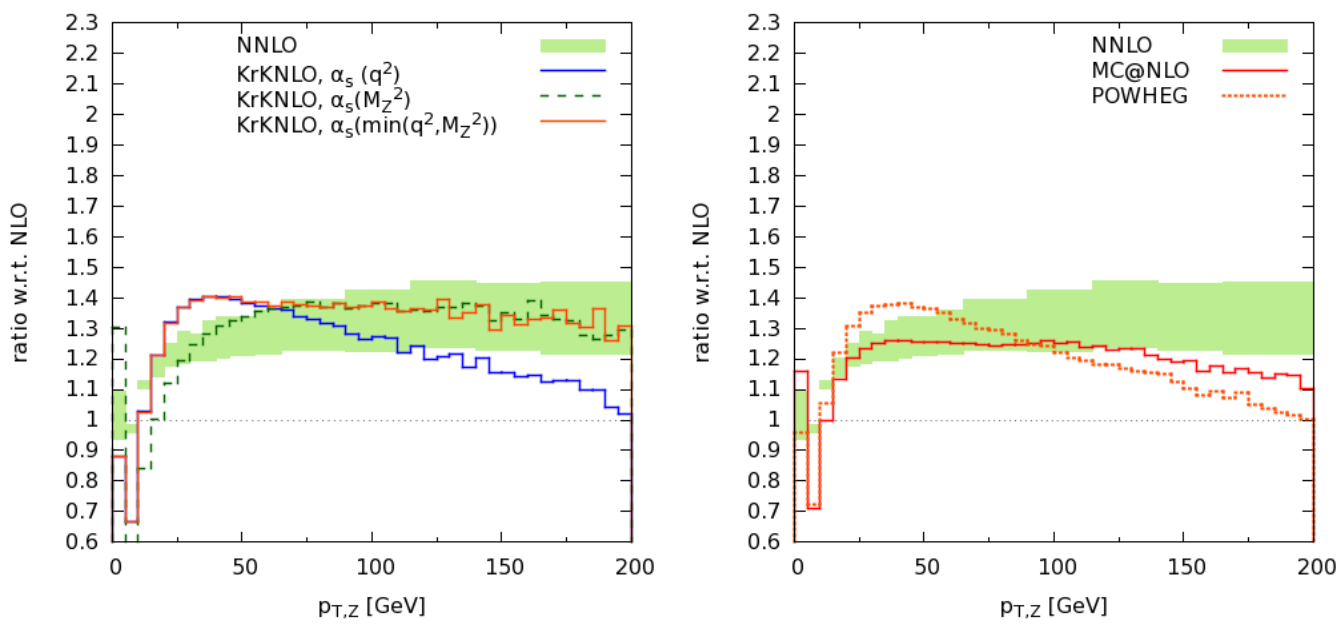

Figure 14. The $Z$-boson transverse-momentum distributions from KrkNLO compared with the fixed-order NNLO result from the DYNNLO program [46] (left). Similar comparisons for POWHEG and MCQNLO are also shown (right). All distributions are divided by the NLO results from MCFM. 


\section{Summary and outlook}

We have discussed the KrkNLO method of matching the LO parton shower with the fixedorder NLO QCD corrections. The method is based on two elements: the change of the factorization scheme from $\overline{\mathrm{MS}}$ to the MC scheme, and upgrading the hardest emission to the full NLO accuracy by reweighting with a simple, positive weight. Details of the method and, in particular, demonstration of its NLO accuracy have been elaborated on in section 3 .

The change of the factorization scheme allows one to eliminate troublesome $z$ dependent terms from the coefficient function and, effectively, it amounts to creating the MC PDFs. In section 4, we have discussed how such PDFs can be obtained and how they differ from the standard $\overline{\mathrm{MS}}$ parton distributions. There, we have also validated the MC factorization scheme by studying the Drell-Yan process at the fixed-order NLO level and showing that the $\overline{\mathrm{MS}}$ and $\mathrm{MC}$ scheme results are identical up to the order $\mathcal{O}\left(\alpha_{s}\right)$.

We have implemented the KrkNLO method on top of the Catani-Seymour type of the parton shower in the Sherpa event generator for the case of the $Z / \gamma^{\star}$-boson production process (hence, the initial-state parton shower). In section 5 , we have presented the comparisons of the NLO-PS matched results obtained with our technique with the fixed-order NLO results from MCFM and with other matched results, namely those of MC@NLO and POWHEG. In particular, we have demonstrated that the KrkNLO results recover the fixedorder NLO predictions (up to sub-percent differences for the $q \bar{q}$ channel only and $\sim 5 \%$ for all channels, coming from the beyond-NLO terms).

As for the comparisons of KrkNLO with MC@NLO and POWHEG at the level of differential distributions, all three methods turn out to give essentially identical results for the $y_{Z}$ spectrum. The $p_{T, Z}$ distributions look somewhat different for each method and the exact features depend on the initial channels and the recoil schemes. In general, the KrkNLO method provides similar predictions to the other two well-established approaches. In particular, the results with both channels and the full parton shower stay very close to those from the POWHEG method implemented in Herwig ++ . Residual differences come from spurious $\mathcal{O}\left(\alpha_{s}^{2}\right)$ terms, which are different in each of the three matching methods.

Applying the KrkNLO method to the Higgs production process is now under development and will be reported in the next paper. Applying the same KrkNLO method to more processes is quite straightforward, in the sense that the modification of the PDFs (to the MC scheme) and subtracting the hard process with the MC scheme counter-term (instead of the $\overline{\mathrm{MS}}$ counter-term) can be done in the usual way for any process, also with more colored particles in the final state. The interesting question is rather whether this method eliminates all the non-physical $\sim \delta\left(p_{T}\right)$ singularities, which are incompatible with the physical phase space of any PS MC? Following the study of ref. [15], we are confident that for color-singlet heavy object production in the $s$ and $t$ channels this is true, although a general formal proof would be welcome. For more colored particles in the final state, this is still an open question. However, it is quite likely that the KrkNLO method reduces significantly the number of such pathological terms, and therefore will facilitate matching parton shower with any NLO and/or NNLO corrected hard processes. This is why, in our opinion, it is definitely worth to pursue this new development path in matching techniques. 
The current methods of the type NNLO+PS [16-18, 45] represent a clear progress in matching of the fixed-order NNLO QCD calculations with PS MCs, but still suffer from various limitations; for instance, they are limited to a certain class of observables only. We are definitely thinking about extending KrkNLO to NNLO+NLOPS, in which NLOPS is a parton shower MC implementing the NLO evolution kernels in the fully exclusive form, thus providing the full set of the soft-collinear counter-terms for the hard process. Ref. [15] reviews several feasibility studies, which show that constructing such a NLOPS is, in principle, plausible. In our opinion, any simplifications of the NLO+PS matching, as in the KrkNLO method, will be instrumental and very useful towards more ambitious fully exclusive NNLO+NLOPS projects.

\section{Acknowledgments}

S.S. is grateful for hospitality to the IFJ PAN in Kraków, where part of this work has been done. We acknowledge useful discussions with Simone Alioli, Stefan Hoeche, Simon Platzer, Gavin Salam, Marek Schoenherr, Steffen Schumann and Alexandra Wilcock. We thank A. Kusina for critical reading the manuscript. We are also grateful to the Cloud Computing for Science and Economy project (CC1) at IFJ PAN (POIG 02.03.03-00-033/09-04) in Kraków whose resources were used to carry out most of the numerical computations for this project. Thanks also to Mariusz Witek and Miłosz Zdybał for their help with CC1. This work was funded in part by the MCnetITN FP7 Marie Curie Initial Training Network PITN-GA-2012-315877.

\section{A First emission in backward evolution}

In case of two LO parton showers in the BEV algorithm the cross section with exactly zero gluons in both showers includes two non-emission $\Delta$-functions

$$
\sigma_{0}^{\mathrm{LO}}=\int d \hat{x}_{F} d \hat{x}_{B} d \Omega \frac{d \sigma}{d \Omega}(\hat{s}, \hat{\theta}) e^{-\Delta_{\mathrm{MC}}^{F}\left(\hat{x}_{F} \mid s, q_{s}^{2}\right)-\Delta_{\mathrm{MC}}^{B}\left(\hat{x}_{B} \mid s, q_{s}^{2}\right)} \bar{D}_{\mathrm{MC}}^{F}\left(\hat{s} \mid s, \hat{x}_{F}\right) \bar{D}_{\mathrm{MC}}^{B}\left(\hat{s} \mid s, \hat{x}_{B}\right),
$$

where $\hat{s}=s \hat{x}_{F} \hat{x}_{B}$. Using the identity of eq. (3.30) twice, the above transforms immediately into

$$
\sigma_{0}^{\mathrm{LO}}=\int d x_{F} d x_{B} d \Omega \frac{d \sigma}{d \Omega}(\hat{s}, \hat{\theta}) e^{-S_{\mathrm{MC}}\left(\hat{s} \mid s, q_{s}^{2}\right)-S_{\mathrm{MC}}\left(\hat{s} \mid s, q_{s}^{2}\right)} \bar{D}_{\mathrm{MC}}^{F}\left(\hat{s} \mid q_{s}^{2}, x_{F}\right), \bar{D}_{\mathrm{MC}}^{B}\left(\hat{s} \mid q_{s}^{2}, x_{B}\right),
$$

which coincides exactly with the $n=0$ result in the forward evolution, starting from $q_{F}^{2}=q_{B}^{2}=q_{s}^{2}$. NB. In the case $n=0$, we identify $\hat{x}_{F}=x_{F}$ and $\hat{x}_{B}=x_{B}$.

In the following we shall simplify the notation omitting the $\hat{s}$ argument in $\bar{D}_{\mathrm{MC}}\left(q^{2}, x\right)=$ $\bar{D}_{\mathrm{MC}}\left(\hat{s} \mid q^{2}, x\right)$ and $S_{\mathrm{MC}}\left(q_{2}^{2}, q_{1}^{2}\right)=S_{\mathrm{MC}}\left(\hat{s} \mid q_{2}^{2}, q_{1}^{2}\right)$, wherever it is unambiguous. 
The distributions from the BEV algorithm with at least one gluon $\left(n_{F}+n_{B}=1,2,3 \ldots\right)$ reads as follows:

$$
\begin{aligned}
\sigma_{1+}^{\mathrm{LO}}= & \int d \hat{x}_{F} d \hat{x}_{B} d \Omega\left\{\int_{q_{s}^{2}}^{s} \frac{d q_{1_{F}}^{2}}{q_{1_{F}}^{2}} \int_{\hat{x}_{F}}^{1} \frac{d z_{1}}{z_{1}} \mathbb{K}_{\mathrm{MC}}\left(\hat{x}_{F} \mid z_{1}, q_{1_{F}}^{2}\right) e^{-\Delta_{\mathrm{MC}}^{F}\left(\hat{x}_{F} \mid s, q_{1_{F}}^{2}\right)-\Delta_{\mathrm{MC}}^{B}\left(\hat{x}_{B} \mid s, q_{1_{F}}^{2}\right)}\right. \\
& \left.+\int_{q_{s}^{2}}^{s} \frac{d q_{1_{B}}^{2}}{q_{1_{B}}^{2}} \int_{\hat{x}_{B}}^{1} \frac{d z_{1}}{z_{1}} \mathbb{K}_{\mathrm{MC}}\left(\hat{x}_{B} \mid z_{1}, q_{1_{B}}^{2}\right) e^{-\Delta_{\mathrm{MC}}^{B}\left(\hat{x}_{B} \mid s, q_{1_{B}}^{2}\right)-\Delta_{\mathrm{MC}}^{F}\left(\hat{x}_{F} \mid s, q_{1_{B}}^{2}\right)}\right\} \\
& \times \bar{D}_{\mathrm{MC}}^{F}\left(\hat{s}, \hat{x}_{F}\right) \bar{D}_{\mathrm{MC}}^{B}\left(\hat{s}, \hat{x}_{B}\right) \frac{d \sigma}{d \Omega}\left(s \hat{x}_{F} \hat{x}_{B}, \hat{\theta}\right),
\end{aligned}
$$

where $z_{1}$ integration limits are imposed by $\hat{s}\left(1-z_{1}\right)^{2} / z_{1}>q_{1}^{2}$ in the $\mathbb{K}$ kernels.

The "unitarity" sum rule $\sigma_{0}^{\mathrm{LO}}+\sigma_{1^{+}}^{\mathrm{LO}}=\sigma^{\mathrm{LO}}$ is of course automatic in the BEV algorithm. Nevertheless, proving it provides an interesting cross-check. The differentiation over the lower boundary in the $q^{2}$ integral defining the $\Delta$-function leads to:

$$
\begin{aligned}
& \frac{\partial}{\partial \ln q^{2}} e^{-\Delta_{\mathrm{MC}}\left(\hat{x}_{F} \mid s, q^{2}\right)-\Delta_{\mathrm{MC}}\left(\hat{x}_{B} \mid s, q^{2}\right)}= \\
& \quad=\int \frac{d z}{z}\left[\mathbb{K}_{\mathrm{MC}}\left(\hat{x}_{F} \mid z, q^{2}\right)+\mathbb{K}_{\mathrm{MC}}\left(\hat{x}_{B} \mid z, q^{2}\right)\right] e^{-\Delta_{\mathrm{MC}}\left(\hat{x}_{F} \mid s, q^{2}\right)-\Delta_{\mathrm{MC}}\left(\hat{x}_{B} \mid s, q^{2}\right)} .
\end{aligned}
$$

After combining two integrals in eq. (A.3) we obtain

$$
\begin{aligned}
& \sigma_{1+}^{\mathrm{LO}}= \int d \hat{x}_{F} d \hat{x}_{B} d \Omega \int_{\ln q_{s}^{2}}^{\ln s} d \ln \left(q_{1}^{2}\right) \frac{\partial}{\partial \ln q_{1}^{2}}\left[e^{-\Delta_{\mathrm{MC}}^{F}\left(\hat{x}_{F} \mid s, q_{1}^{2}\right)-\Delta_{\mathrm{MC}}^{B}\left(\hat{x}_{B} \mid s, q_{1}^{2}\right)}\right] \\
& \times \frac{d \sigma}{d \Omega}\left(s \hat{x}_{F} \hat{x}_{B}, \hat{\theta}\right) \bar{D}_{\mathrm{MC}}^{F}\left(s, \hat{x}_{F}\right) \bar{D}_{\mathrm{MC}}^{B}\left(s, \hat{x}_{B}\right) \\
&=\int d \hat{x}_{F} d \hat{x}_{B} d \Omega\left[1-e^{-\Delta_{\mathrm{MC}}^{F}\left(\hat{x}_{F} \mid s, q_{s}^{2}\right)-\Delta_{\mathrm{MC}}^{B}\left(\hat{x}_{B} \mid s, q_{s}^{2}\right)}\right] \frac{d \sigma}{d \Omega}\left(s \hat{x}_{F} \hat{x}_{B}, \hat{\theta}\right) \bar{D}_{\mathrm{MC}}^{F}\left(s, \hat{x}_{F}\right) \bar{D}_{\mathrm{MC}}^{B}\left(s, \hat{x}_{B}\right),
\end{aligned}
$$

from which the sum rule $\sigma_{0}^{\mathrm{LO}}+\sigma_{1^{+}}^{\mathrm{LO}}=\sigma^{\mathrm{LO}}$ results immediately.

Before we transform eq. (A.3) into the FEV picture, let us first expand $\mathbb{K}$-kernels: ${ }^{32}$

$$
\begin{aligned}
& \sigma_{1+}^{\mathrm{LO}}=\int d \hat{x}_{F} d \hat{x}_{B} d \Omega \\
& \quad \times\left\{\int_{q_{s}^{2}}^{s} \frac{d q_{1_{F}}^{2}}{q_{1_{F}}^{2}} \int_{x_{F}}^{1} \frac{d z_{1}}{z_{1}} \theta_{\hat{s}\left(1-z_{1}\right)^{2} / z_{1}>q_{1_{F}}^{2}} \mathcal{P}\left(z_{1}\right) e^{-\Delta_{\mathrm{MC}}^{F}\left(\hat{x}_{F} \mid s, q_{1_{F}}^{2}\right)} e^{-\Delta_{\mathrm{MC}}^{B}\left(\hat{x}_{B} \mid s, q_{1_{F}}^{2}\right)} \frac{\bar{D}_{\mathrm{MC}}^{F}\left(q_{1_{F}}^{2}, \frac{\hat{x}_{F}}{z_{1}}\right)}{\bar{D}_{\mathrm{MC}}^{F}\left(q_{1_{F}}^{2}, \hat{x}_{F}\right)}\right. \\
& \left.\quad+\int_{q_{s}^{2}}^{s} \frac{d q_{1_{B}}^{2}}{q_{1_{B}}^{2}} \int_{x_{B}}^{1} \frac{d z_{1}}{z_{1}} \theta_{\hat{s}\left(1-z_{1}\right)^{2} / z_{1}>q_{1_{B}}^{2}} \mathcal{P}\left(z_{1}\right) e^{-\Delta_{\mathrm{MC}}^{F}\left(\hat{x}_{F} \mid s, q_{1_{B}}^{2}\right)} e^{-\Delta_{\mathrm{MC}}^{B}\left(\hat{x}_{B} \mid s, q_{1_{B}}^{2}\right)} \frac{\bar{D}_{\mathrm{MC}}^{B}\left(q_{1_{B}}^{2}, \frac{\hat{x}_{B}}{z_{1}}\right)}{\bar{D}_{\mathrm{MC}}^{B}\left(q_{1_{B}}^{2}, \hat{x}_{F}\right)}\right\} \\
& \quad \times \bar{D}_{\mathrm{MC}}^{F}\left(s, \hat{x}_{F}\right) \bar{D}_{\mathrm{MC}}^{B}\left(s, \hat{x}_{B}\right) \frac{d \sigma}{d \Omega}\left(s \hat{x}_{F} \hat{x}_{B}, \hat{\theta}\right) .
\end{aligned}
$$

\footnotetext{
${ }^{32}$ We denote $\mathcal{P}(z)=\frac{C_{F} \alpha_{s}}{\pi} \frac{\bar{P}(z)}{1-z}$
} 
Now using again the identities

$e^{-\Delta_{\mathrm{MC}}^{F}\left(\hat{x}_{F} \mid s, q_{1_{F}}^{2}\right)}=e^{-S_{\mathrm{MC}}^{F}\left(s, q_{1_{F}}^{2}\right)} \frac{\bar{D}_{\mathrm{MC}}^{F}\left(q_{1_{F}}^{2}, \hat{x}_{F}\right)}{\bar{D}_{\mathrm{MC}}^{F}\left(s, \hat{x}_{F}\right)}, e^{-\Delta_{\mathrm{MC}}^{B}\left(\hat{x}_{B} \mid s, q_{1_{F}}^{2}\right)}=e^{-S_{\mathrm{MC}}^{B}\left(s, q_{1_{F}}^{2}\right)} \frac{\bar{D}_{\mathrm{MC}}^{B}\left(q_{1_{F}}^{2}, \hat{x}_{B}\right)}{\bar{D}_{\mathrm{MC}}^{B}\left(s, \hat{x}_{F}\right)}$,

we arrive at the following FEV representation

$$
\begin{aligned}
& \sigma_{1+}^{\mathrm{LO}}=\int d \hat{x}_{F} d \hat{x}_{B} d \Omega \frac{d \sigma}{d \Omega}\left(s \hat{x}_{F} \hat{x}_{B}, \hat{\theta}\right) \\
& \times\left\{\int_{q_{s}^{2}}^{s} \frac{d q_{1_{F}}^{2}}{q_{1_{F}}^{2}} \int_{x_{F}}^{1} \frac{d z_{1}}{z_{1}} \theta_{\hat{s}\left(1-z_{1}\right)^{2} / z_{1}>q_{1_{F}}^{2}} \mathcal{P}\left(z_{1}\right) e^{-S_{\mathrm{MC}}^{F}\left(s, q_{1_{F}}^{2}\right)-S_{\mathrm{MC}}^{B}\left(s, q_{1_{F}}^{2}\right)} D_{\mathrm{MC}}^{F}\left(q_{1_{F}}^{2}, \frac{\hat{x}_{F}}{z_{1}}\right) D_{\mathrm{MC}}^{B}\left(q_{1_{F}}^{2}, \hat{x}_{B}\right)\right. \\
& \left.+\int_{q_{s}^{2}}^{s} \frac{d q_{1_{B}}^{2}}{q_{1_{B}}^{2}} \int_{x_{B}}^{1} \frac{d z_{1}}{z_{1}} \theta_{\hat{s}\left(1-z_{1}\right)^{2} / z_{1}>q_{1_{B}}^{2}} \mathcal{P}\left(z_{1}\right) e^{-S_{\mathrm{MC}}^{F}\left(s, q_{1_{B}}^{2}\right)-S_{\mathrm{MC}}^{B}\left(s, q_{1_{B}}^{2}\right)} D_{\mathrm{MC}}^{F}\left(q_{1_{B}}^{2}, \hat{x}_{F}\right) D_{\mathrm{MC}}^{B}\left(q_{1_{B}}^{2}, \frac{\hat{x}_{B}}{z_{1}}\right)\right\} .
\end{aligned}
$$

Finally, the longitudinal integrations are streamlined with the help of the substitutions to the $x$-variables before the emission, $x_{F} / z_{1} \rightarrow x_{F}, x_{1_{B}}=x_{B}$ or $x_{B} / z_{1} \rightarrow x_{B}, x_{1_{F}}=x_{B}$, respectively in each shower, and the convolution structure is made manifestly symmetric:

$$
\begin{aligned}
& \sigma_{1+}^{\mathrm{LO}}=\int d x \int d x_{F} d x_{B} d \Omega \\
& \times\left\{\int_{q_{s}^{2}}^{s} \frac{d q_{1_{F}}^{2}}{q_{1_{F}}^{2}} \int_{x_{F}}^{1} \frac{d z_{1}}{z_{1}} \theta_{\hat{s}\left(1-z_{1}\right)^{2} / z_{1}>q_{1_{F}}^{2}} \mathcal{P}\left(z_{1}\right) e^{-S_{\mathrm{MC}}^{F}\left(s, q_{1_{F}}^{2}\right)-S_{\mathrm{MC}}^{B}\left(s, q_{1_{F}}^{2}\right)} \bar{D}_{\mathrm{MC}}^{F}\left(q_{1_{F}}^{2}, x_{F}\right) \bar{D}_{\mathrm{MC}}^{B}\left(q_{1_{F}}^{2}, x_{B}\right)\right. \\
& \left.+\int_{q_{s}^{2}}^{s} \frac{d q_{1_{B}}^{2}}{q_{1_{B}}^{2}} \int_{x_{B}}^{1} \frac{d z_{1}}{z_{1}} \theta_{\hat{s}\left(1-z_{1}\right)^{2} / z_{1}>q_{1_{B}}^{2}} \mathcal{P}\left(z_{1}\right) e^{-S_{\mathrm{MC}}^{F}\left(s, q_{1_{B}}^{2}\right)-S_{\mathrm{MC}}^{B}\left(s, q_{1_{B}}^{2}\right)} \bar{D}_{\mathrm{MC}}^{F}\left(q_{1_{B}}^{2}, x_{F}\right) \bar{D}_{\mathrm{MC}}^{B}\left(q_{1_{B}}^{2}, x_{B}\right)\right\} \\
& \times \frac{d \sigma}{d \Omega}\left(s x_{F} x_{B}, \hat{\theta}\right) \delta\left(x-z_{1} x_{F} x_{B}\right)
\end{aligned}
$$

The above formula is for the FEV algorithm but for one gluon next to the hard process and any number of trailing gluons down to $q_{s}^{2}$. The same formula in eq. (3.33) was obtained by means of factorizing such a gluon from the fully exclusive FEV formula of eq. (3.24).

\section{B Exclusive NLO corrections in $\overline{\mathrm{MS}}$ scheme}

Following ref. [8], the bare NLO real + virtual unintegrated DY cross section for the incoming $q \bar{q}$ with the effective mass of $\sqrt{s_{1}}$, in $d=4+2 \epsilon$ dimensions, is quite simple:

$$
\begin{aligned}
\sigma_{q \bar{q}, B}^{\mathrm{NLO}}\left(s_{1}, \epsilon\right) & =\int d \Omega \int_{\alpha+\beta \leq 1} d \alpha d \beta\left\{\delta(\alpha) \delta(\beta) \mathcal{W}_{0}\left(s_{1}\right)+\mathcal{W}_{2}\left(s_{1}, \alpha, \beta\right)\right\}, \\
\mathcal{W}_{0}\left(s_{1}\right) & =\left(1+V\left(s_{1}, \epsilon\right)\right) \frac{d \sigma_{0}}{d \Omega}\left(s_{1}, \theta\right), \\
\mathcal{W}_{2}\left(s_{1}, \alpha, \beta\right) & =H_{2}\left(s_{1}, \alpha, \beta, \epsilon\right) \frac{d \sigma_{0}}{d \Omega}\left(z s_{1}, \theta_{F}\right)+H_{2}\left(s_{1}, \beta, \alpha, \epsilon\right) \frac{d \sigma_{0}}{d \Omega}\left(z s_{1}, \theta_{B}\right)
\end{aligned}
$$




$$
\begin{aligned}
V\left(s_{1}, \epsilon\right) & =\frac{2 C_{F} \alpha_{s}}{\pi} \frac{(4 \pi)^{-\epsilon} \Gamma(1+\epsilon)}{\Gamma(1+2 \epsilon)}\left(\frac{s_{1}}{\mu^{2}}\right)^{\epsilon}\left(-\frac{1}{2 \epsilon^{2}}+\frac{3}{4} \frac{1}{\epsilon}-2-\frac{\pi^{2}}{12}\right), \\
H_{2}\left(s_{1}, \alpha, \beta, \epsilon\right) & =\frac{2 C_{F} \alpha_{s}}{\pi} \frac{(4 \pi)^{-\epsilon}}{\Gamma(1+\epsilon)}\left(\frac{s_{1} \alpha \beta}{\mu^{2}}\right)^{\epsilon} \frac{(1-\alpha)^{2}+\epsilon \alpha^{2}}{2 \alpha \beta}
\end{aligned}
$$

where $z=1-\alpha-\beta$ and notation of section 3.1 is used. The integration over the azimuthal angle is already done in order to improve readability. The integration decay lepton angles and $\alpha$ and $\beta$ keeping $z=1-\alpha-\beta$ fixed, leads to a divergent (bare) result

$$
\begin{aligned}
\sigma_{q \bar{q}, B}^{\mathrm{NLO}}\left(s_{1}, \epsilon\right)= & \int_{0}^{1} d z \sigma_{0}\left(s_{1} z\right)\left[\delta(1-z)+\rho_{B}\left(z, s_{1}, \epsilon\right)\right] \\
\rho_{B}\left(z, s_{1}, \epsilon\right)= & \frac{2 \alpha_{s}}{\pi} C_{F} \frac{(4 \pi)^{-\epsilon}}{\Gamma(1+\epsilon)}\left(\frac{s_{1}}{\mu^{2}}\right)^{\epsilon}\left\{\left(\frac{\pi^{2}}{6}-2\right) \delta(1-z)\right. \\
& \left.-\frac{1}{\epsilon}\left(\frac{1+z^{2}}{2(1-z)}\right)_{+}+\left(1+z^{2}\right)\left(\frac{\ln (1-z)}{1-z}\right)_{+}\right\} .
\end{aligned}
$$

The $\overline{\mathrm{MS}}$ recipe tells us to subtract from the bare radiator function $\rho_{B}$ its pole part

$$
2 \Gamma_{\overline{\mathrm{MS}}}^{[1]}(\epsilon)=\mathrm{PP} \rho_{B}\left(z, s_{1}, \epsilon\right)=\frac{2 \alpha_{s}}{\pi} C_{F} \frac{1}{\epsilon} \frac{(4 \pi)^{-\epsilon}}{\Gamma(1+\epsilon)}\left(\frac{1+z^{2}}{2(1-z)}\right)_{+} .
$$

After including PDFs and integrating over $s_{1}$ which leads to the replacement $s_{1} \rightarrow s x_{F} x_{B}$, we obtain the finite hadron-level cross section

$$
\begin{aligned}
\sigma^{\mathrm{NLO}}(s)= & \int d x_{F} d x_{B} d z\{\delta(1-z) \\
& \left.+\left[(1-\mathrm{PP}) \rho_{B}\left(z, s x_{F} x_{B}, \epsilon\right)\right]\right\} \sigma_{0}\left(s z x_{F} x_{B}\right) D_{q}^{\overline{\mathrm{MS}}}\left(\mu^{2}, x_{F}\right) D_{\bar{q}}^{\overline{\mathrm{MS}}}\left(\mu^{2}, x_{B}\right)
\end{aligned}
$$

The contribution from the $q \bar{q}$ channel to the inclusive coefficient function of the Drell-Yan process is ${ }^{33} C_{2 q}^{\overline{\mathrm{MS}}}(z)=\left.(1-\mathrm{PP}) \rho_{B}(z, \epsilon)\right|_{\mu^{2}=z s_{1}}$ of eq. (3.11). The above formula can be used to define the PDFs and dig them out from experimental DY data. Alternatively, it can be used to predict the DY cross section taking the PDFs obtained from other processes, e.g. deep inelastic lepton-proton scattering (DIS).

The above formulation is suitable for the total cross section. In the case of any NLOclass exclusive observable defined by the function $J=J_{\mathrm{NLO}}\left(x_{F}, x_{B}, z, k_{1 T}^{2}\right)$ of eq. (3.35), the above formulas are reorganized using the technique of the Catani-Seymour soft-collinear counter-terms (SCC). In the present case SCC coincides (for $\varepsilon=0$ ) with the single gluon LO distribution of the parton shower MC:

$$
\mathcal{W}_{c t}\left(s_{1}, \alpha, \beta, \epsilon\right)=\frac{2 C_{F} \alpha_{s}}{\pi} \frac{(4 \pi)^{-\epsilon}}{\Gamma(1+\epsilon)}\left(\frac{s_{1} \alpha \beta}{\mu^{2}}\right)^{\epsilon} \frac{1+z^{2}+\varepsilon(1-z)^{2}}{2 \alpha \beta} \frac{d \sigma_{0}}{d \Omega}\left(z s_{1}, \theta\right)
$$

\footnotetext{
${ }^{33}$ The assignment $\mu^{2}=z s_{1}$ induces the presence of the term $\sim \ln (z)\left(1+z^{2}\right) /(1-z)$ in $C_{2 q}^{\overline{\mathrm{MS}}}$.
} 
where $z=1-\alpha-\beta$. The above SCC is subtracted and added:

$$
\begin{aligned}
& \sigma^{\mathrm{NLO}}(s)[J]=\int d x_{F} d x_{B} D_{q}^{\overline{\mathrm{MS}}}\left(\mu^{2}, x_{F}\right) D_{\bar{q}}^{\overline{\mathrm{MS}}}\left(\mu^{2}, x_{B}\right) \\
& \times\left\{(1-\mathrm{PP})\left[\int d \Omega \mathcal{W}_{0}\left(s_{1}\right)+\int_{\alpha+\beta \leq 1} d \alpha d \beta \int d \Omega \mathcal{W}_{c t}\left(s_{1}, \alpha, \beta, \epsilon\right)\right] J\left(x_{F}, x_{B}, 1,0\right)\right. \\
& \left.\quad+\int d \Omega \int d \alpha d \beta\left[\mathcal{W}_{2}\left(s_{1}, \alpha, \beta\right) J\left(x_{F}, x_{B}, z, k_{1 T}^{2}\right)-\mathcal{W}_{c t}\left(s_{1}, \alpha, \beta\right) J\left(x_{F}, x_{B}, 1,0\right)\right]_{\epsilon=0}\right\},
\end{aligned}
$$

where $s_{1} \equiv s x_{F} x_{B}$. In the second real emission integral $\mathcal{W}_{c t}$ eliminates completely the soft/collinear singularities, so it can be evaluated in the $\varepsilon \rightarrow 0$ limit. The key point here is that $\mathcal{W}_{c t}$ is multiplied by $J_{\mathrm{LO}}=J\left(x_{F}, x_{B}, 1,0\right)$, hence the partial integration $\int d \alpha d \beta \delta(z-$ $\alpha-\beta)$ can be performed for the fixed $z$ in order to isolate and subtract the $1 / \epsilon$ pole:

$$
\begin{aligned}
\sigma^{\mathrm{NLO}}(s)[J]= & \int d x_{F} d x_{B} d \Omega \int d z D_{q}^{\overline{\mathrm{MS}}}\left(\mu^{2}, x_{F}\right) D_{\bar{q}}^{\overline{\mathrm{MS}}}\left(\mu^{2}, x_{B}\right) \\
& \times\left\{\left(1+\Delta_{V S}^{q \bar{q}}\right) \delta(1-z)+2 \Sigma_{q}(z)\right\} \frac{d \sigma_{0}}{d \Omega}\left(z s_{1}, \theta\right) J\left(x_{F}, x_{B}, 1,0\right) \\
& +\int d x_{F} d x_{B} d \Omega D_{q}^{\overline{\mathrm{MS}}}\left(\mu^{2}, x_{F}\right) D_{\bar{q}}^{\overline{\mathrm{MS}}}\left(\mu^{2}, x_{B}\right) \int_{\alpha+\beta \leq 1} d \alpha d \beta \Delta \mathcal{W}_{\text {real }}[J],
\end{aligned}
$$

with

$$
\begin{aligned}
2 \Sigma_{q}(z) & =\frac{2 C_{F} \alpha_{s}}{\pi}\left\{\frac{1+z^{2}}{2(1-z)} \ln \frac{(1-z)^{2}}{z}+\frac{1+z^{2}}{2(1-z)} \ln \frac{\hat{s}}{\mu^{2}}+\frac{1-z}{2}\right\}_{+}, \\
\Delta \mathcal{W}_{\text {real }}[J] & =\left[\mathcal{W}_{2}\left(s_{1}, \alpha, \beta\right) J\left(x_{F}, x_{B}, z, k_{1 T}^{2}\right)-\mathcal{W}_{c t}\left(s_{1}, \alpha, \beta\right) J\left(x_{F}, x_{B}, 1,0\right)\right]_{\epsilon=0},
\end{aligned}
$$

where $\Delta_{V S}^{q \bar{q}}=\frac{2 C_{F} \alpha_{s}}{\pi}\left(\frac{\pi^{2}}{3}-\frac{5}{8}\right)$ is that of eq. (3.10), $\left.\Sigma_{q}(z)\right|_{\hat{s}=\mu^{2}}=\Delta C_{2 q}$, see eq. (3.13), and $\hat{s}=s z x_{F} x_{B}$ as usual.

In the MC scheme the entire $\sim \delta\left(k_{1 T}^{2}\right) \Sigma(z)$ part gets eliminated (modulo $\mathcal{O}\left(\alpha_{s}^{2}\right)$ terms) thanks to the assignment $\hat{s}=\mu^{2}$ and redefinition of the PDFs

$$
D_{q, \bar{q}}^{\mathrm{MC}}\left(\mu^{2}, x\right)=\int d z d x^{\prime} \delta\left(x-z x^{\prime}\right)\left[\delta(1-z)+\Sigma_{q}(z)\right]_{\hat{s}=\mu^{2}} D_{q, \bar{q}}^{\overline{\mathrm{MS}}}\left(\mu^{2}, x^{\prime}\right),
$$

see eq. (3.17). The only freedom ${ }^{34}$ is in the coefficient of $\delta(1-z)$. However, the convention $\int d z \Sigma(z)=0$ adopted in ref. [19] removes this freedom and fixes the content of $\Delta_{V S}$.

Inserting eq. (B.9) into eq. (B.7), we obtain the formula for the NLO cross section in the MC scheme

$$
\begin{aligned}
\sigma_{\mathrm{MC}}^{\mathrm{NLO}}(s)[J]= & \int d x_{F} d x_{B} d \Omega \int d z D_{q}^{\mathrm{MC}}\left(\hat{s}, x_{F}\right) D_{\bar{q}}^{\mathrm{MC}}\left(\hat{s}, x_{B}\right) \\
& \times\left(1+\Delta_{V S}^{q \bar{q}}\right) \frac{d \sigma_{0}}{d \Omega}\left(s_{1}, \theta\right) J\left(x_{F}, x_{B}, 1,0\right) \\
& +\int d x_{F} d x_{B} d \Omega \int_{\alpha+\beta \leq 1} d \alpha d \beta \Delta \mathcal{W}_{\text {real }}[J] D_{q}^{\mathrm{MC}}\left(\hat{s}, x_{F}\right) D_{\bar{q}}^{\mathrm{MC}}\left(\hat{s}, x_{B}\right),
\end{aligned}
$$

\footnotetext{
${ }^{34}$ The $z \neq 0$ part of $\Sigma$ is unique - it has to be eliminate completely from the NLO distribution, because the singular non-positive $\sim \delta\left(k_{1 T}^{2}\right) \Sigma$ term cannot be included in the multiplicative MC weight.
} 
where we have replaced the $\overline{\mathrm{MS}} \mathrm{PDF}$ by the MC PDFs also in the real part. The cross sections in eqs. (B.10) and (B.7) are equal up to $\mathcal{O}\left(\alpha_{s}^{2}\right)$.

Finally, let us remark, following ref. [19], that formally the transition from the $\overline{\mathrm{MS}}$ to MC scheme can be done by means of subtracting from the bare NLO distribution the term

$$
\Gamma_{\mathrm{MC}}^{[1]}(\epsilon)=\Gamma_{\overline{\mathrm{MS}}}^{[1]}(\epsilon)+\left.\Sigma_{q}(z)\right|_{\hat{s}=\mu^{2}},
$$

instead of the pure pole $\Gamma_{\mathrm{MS}}^{[1]}(\epsilon)$. It is appealing, that $\Gamma_{\mathrm{MC}}^{[1]}(\epsilon)$ concides with the integrated single-gluon emission distribution of PS MC extrapolated to $d=4+2 \epsilon$ dimensions. This justifies the name of the MC factorization scheme.

Open Access. This article is distributed under the terms of the Creative Commons Attribution License (CC-BY 4.0), which permits any use, distribution and reproduction in any medium, provided the original author(s) and source are credited.

\section{References}

[1] A. Buckley et al., General-purpose event generators for LHC physics, Phys. Rept. 504 (2011) 145 [arXiv:1101.2599] [INSPIRE].

[2] S. Frixione and B.R. Webber, Matching NLO QCD computations and parton shower simulations, JHEP 06 (2002) 029 [hep-ph/0204244] [INSPIRE].

[3] P. Nason, A New method for combining NLO QCD with shower Monte Carlo algorithms, JHEP 11 (2004) 040 [hep-ph/0409146] [INSPIRE].

[4] P. Nason and B. Webber, Next-to-Leading-Order Event Generators, Ann. Rev. Nucl. Part. Sci. 62 (2012) 187 [arXiv: 1202.1251] [INSPIRE].

[5] M.H. Seymour, A Simple prescription for first order corrections to quark scattering and annihilation processes, Nucl. Phys. B 436 (1995) 443 [hep-ph/9410244] [INSPIRE].

[6] J. Andre and T. Sjöstrand, A Matching of matrix elements and parton showers, Phys. Rev. D 57 (1998) 5767 [hep-ph/9708390] [INSPIRE].

[7] S. Catani, F. Krauss, R. Kuhn and B.R. Webber, QCD matrix elements + parton showers, JHEP 11 (2001) 063 [hep-ph/0109231] [INSPIRE].

[8] G. Altarelli, R.K. Ellis and G. Martinelli, Large Perturbative Corrections to the Drell-Yan Process in QCD, Nucl. Phys. B 157 (1979) 461 [inSPIRE].

[9] J. Bellm et al., HERWIG++ 2.7 Release Note, arXiv:1310.6877 [INSPIRE].

[10] T. Sjöstrand et al., An Introduction to PYTHIA 8.2, Comput. Phys. Commun. 191 (2015) 159 [arXiv: 1410.3012] [INSPIRE].

[11] T. Gleisberg et al., Event generation with SHERPA 1.1, JHEP 02 (2009) 007 [arXiv:0811.4622] [INSPIRE].

[12] Z. Nagy and D.E. Soper, A parton shower based on factorization of the quantum density matrix, JHEP 06 (2014) 097 [arXiv: 1401.6364] [INSPIRE].

[13] C. Anastasiou, L.J. Dixon, K. Melnikov and F. Petriello, High precision QCD at hadron colliders: Electroweak gauge boson rapidity distributions at NNLO, Phys. Rev. D 69 (2004) 094008 [hep-ph/0312266] [INSPIRE]. 
[14] G. Bozzi, S. Catani, D. de Florian and M. Grazzini, Higgs boson production at the LHC: Transverse-momentum resummation and rapidity dependence, Nucl. Phys. B 791 (2008) 1 [arXiv:0705.3887] [INSPIRE].

[15] S. Jadach, A. Kusina, W. Płaczek and M. Skrzypek, NLO corrections in the initial-state parton shower Monte Carlo, Acta Phys. Polon. B 44 (2013) 2179 [arXiv:1310.6090] [INSPIRE].

[16] K. Hamilton, P. Nason, C. Oleari and G. Zanderighi, Merging $H / W / Z+O$ and 1 jet at $N L O$ with no merging scale: a path to parton shower + NNLO matching, JHEP 05 (2013) 082 [arXiv: 1212.4504] [INSPIRE].

[17] S. Höche, Y. Li and S. Prestel, Drell-Yan lepton pair production at NNLO QCD with parton showers, Phys. Rev. D 91 (2015) 074015 [arXiv:1405.3607] [INSPIRE].

[18] S. Alioli, C.W. Bauer, C. Berggren, F.J. Tackmann, J.R. Walsh and S. Zuberi, Matching Fully Differential NNLO Calculations and Parton Showers, JHEP 06 (2014) 089 [arXiv: 1311.0286] [INSPIRE].

[19] S. Jadach, A. Kusina, W. Placzek, M. Skrzypek and M. Slawinska, Inclusion of the QCD next-to-leading order corrections in the quark-gluon Monte Carlo shower, Phys. Rev. D 87 (2013) 034029 [arXiv:1103.5015] [INSPIRE].

[20] S. Jadach, M. Jezabek, A. Kusina, W. Placzek and M. Skrzypek, NLO corrections to hard process in QCD shower - proof of concept, Acta Phys. Polon. B 43 (2012) 2067 [arXiv: 1209.4291] [INSPIRE].

[21] M. Bahr et al., HERWIG++ Physics and Manual, Eur. Phys. J. C 58 (2008) 639 [arXiv: 0803.0883] [INSPIRE].

[22] S. Gieseke, C. Rohr and A. Siodmok, Colour reconnections in HERWIG++, Eur. Phys. J. C 72 (2012) 2225 [arXiv:1206.0041] [INSPIRE].

[23] S. Platzer and S. Gieseke, Coherent Parton Showers with Local Recoils, JHEP 01 (2011) 024 [arXiv: 0909.5593] [INSPIRE].

[24] S. Catani and M.H. Seymour, A General algorithm for calculating jet cross-sections in NLO QCD, Nucl. Phys. B 485 (1997) 291 [Erratum ibid. B 510 (1998) 503] [hep-ph/9605323] [INSPIRE].

[25] S. Alioli, K. Hamilton and E. Re, Practical improvements and merging of POWHEG simulations for vector boson production, JHEP 09 (2011) 104 [arXiv:1108.0909] [INSPIRE].

[26] Z. Nagy and D.E. Soper, Parton showers with quantum interference: Leading color, with spin, JHEP 07 (2008) 025 [arXiv: 0805.0216] [INSPIRE].

[27] Z. Nagy and D.E. Soper, Parton showers with quantum interference: Leading color, spin averaged, JHEP 03 (2008) 030 [arXiv:0801.1917] [INSPIRE].

[28] L.N. Lipatov, The parton model and perturbation theory, Sov. J. Nucl. Phys. 20 (1975) 94.

[29] V.N. Gribov and L.N. Lipatov, Deep inelastic e p scattering in perturbation theory, Sov. J. Nucl. Phys. 15 (1972) 438 [inSPIRE].

[30] G. Altarelli and G. Parisi, Asymptotic freedom in parton language, Nucl. Phys. 126 (1977) 298. 
[31] Yu. L. Dokshitzer, Calculation of structure functions of deep-inelastic scattering and $e^{+} e^{-}$ annihilation by perturbation theory in quantum chromodynamics, Sov. Phys. JETP 46 (1977) 641.

[32] J.M. Campbell and R.K. Ellis, An Update on vector boson pair production at hadron colliders, Phys. Rev. D 60 (1999) 113006 [hep-ph/9905386] [INSPIRE].

[33] F.A. Berends and R. Kleiss, Initial State Radiation for $e^{+} e^{-}$Annihilation Into Jets, Nucl. Phys. B 178 (1981) 141 [INSPIRE].

[34] J.C. Collins and D.E. Soper, Angular Distribution of Dileptons in High-Energy Hadron Collisions, Phys. Rev. D 16 (1977) 2219 [INSPIRE].

[35] S. Frixione, P. Nason and C. Oleari, Matching NLO QCD computations with Parton Shower simulations: the POWHEG method, JHEP 11 (2007) 070 [arXiv: 0709. 2092] [INSPIRE].

[36] K. Golec-Biernat, S. Jadach, W. Płaczek and M. Skrzypek, Solving QCD evolution equations in rapidity space with Markovian Monte Carlo, Acta Phys. Polon. B 39 (2008) 115 [Erratum ibid. 40 (2009) 213] [arXiv:0708.1906] [InSPIRE].

[37] S. Jadach, B.F.L. Ward and Z. Was, The Precision Monte Carlo event generator K K for two fermion final states in $e^{+} e^{-}$collisions, Comput. Phys. Commun. 130 (2000) 260 [hep-ph/9912214] [INSPIRE].

[38] G. Altarelli and M L. Mangano eds., Proccedings of the Workshop on Standard Model Physics (and More) at the LHC, Yellow Report, CERN 2000-04 (2000).

[39] A.D. Martin, W.J. Stirling, R.S. Thorne and G. Watt, Parton distributions for the LHC, Eur. Phys. J. C 63 (2009) 189 [arXiv:0901.0002] [InSPIRE].

[40] S. Schumann and F. Krauss, A Parton shower algorithm based on Catani-Seymour dipole factorisation, JHEP 03 (2008) 038 [arXiv:0709.1027] [INSPIRE].

[41] S. Hoeche, F. Krauss, S. Schumann and F. Siegert, QCD matrix elements and truncated showers, JHEP 05 (2009) 053 [arXiv:0903.1219] [INSPIRE].

[42] S. Platzer and S. Gieseke, Dipole Showers and Automated NLO Matching in HERWIG++, Eur. Phys. J. C 72 (2012) 2187 [arXiv:1109.6256] [InSPIRE].

[43] S. Platzer, ExSample: A Library for Sampling Sudakov-Type Distributions, Eur. Phys. J. C 72 (2012) 1929 [arXiv:1108.6182] [INSPIRE].

[44] S. Hoeche, S. Schumann and F. Siegert, Hard photon production and matrix-element parton-shower merging, Phys. Rev. D 81 (2010) 034026 [arXiv:0912.3501] [INSPIRE].

[45] S. Höche and S. Prestel, The midpoint between dipole and parton showers, Eur. Phys. J. C 75 (2015) 461 [arXiv: 1506. 05057] [INSPIRE].

[46] S. Catani, L. Cieri, G. Ferrera, D. de Florian and M. Grazzini, Vector boson production at hadron colliders: a fully exclusive QCD calculation at NNLO, Phys. Rev. Lett. 103 (2009) 082001 [arXiv: 0903.2120] [inSPIRE]. 